\title{
Temporal filters in response to presynaptic spike trains: Interplay of cellular, synaptic and short-term plasticity time scales
}

\author{
Yugarshi Mondal ${ }^{1}$, Rodrigo F. O. Pena ${ }^{2}$, Horacio G. Rotstein ${ }^{2}{ }^{\dagger}$ \\ ${ }^{1}$ Department of Mathematics and Statistics \\ Stony Brook University, USA \\ ${ }^{2}$ Federated Department of Biological Sciences \\ New Jersey Institute of Technology and Rutgers University, USA
}

September 16, 2021

\begin{abstract}
Temporal filters, the ability of postsynaptic neurons to preferentially select certain presynaptic input patterns, have been shown to be associated with the notion of information filtering and coding of sensory inputs. Their properties can be dynamically characterized as the transient responses to periodic presynaptic inputs. Short-term plasticity (STP) has been proposed to be an important player in the generation of temporal filters, but the response of postsynaptic neurons to presynaptic inputs depends on a collection of time scales in addition to STP's, which conspire to create temporal filters: the postsynaptic time scales generated by the cellular intrinsic currents and the presynaptic time scales captured by the ISI distribution patterns. The mechanisms by which these time scales and the processes giving rise to them interact to produce temporal filters in response to presynaptic input spike trains are not well understood. We carry out a systematic modeling and computational analysis to understand how the postsynaptic low-, high- and band-pass temporal filters are generated in response to periodic presynaptic spike trains in the presence STP, and how the dynamic properties of these filters depend on the interplay of a hierarchy of processes: arrival of the presynaptic spikes, the activation of STP and its effect on the synaptic connection efficacy, and the response of the postsynaptic cell. The time scales associated with each of these processes operate at the short-term, single-event level (they are activated at the arrival of each presynaptic spike) and collectively produce the long-term time scales that determine the shape and properties of the filters. We develop a series of mathematical tools to address these issues for a relatively simple model where depression and facilitation interact only at the level of the synaptic efficacy change as time progresses and we extend our results and tools to account for more complex models that involve interactions at the STP level and multiple STP time scales. We use these tools to understand the mechanisms of generation of temporal filters in the postsynaptic cells in terms of the properties and dynamics of the interacting building blocks.
\end{abstract}

${ }^{*}$ Current address: Volen Center for Complex Systems, Brandeis University, Waltham, MA, USA

${ }^{\dagger}$ E-mail: horacio@njit.edu, corresponding author

${ }^{\ddagger}$ Graduate Faculty, Behavioral Neurosciences Program, Rutgers University; Corresponding Investigator, CONICET, Argentina. 


\section{Introduction}

The synaptic communication between neurons involves a multiplicity of interacting time scales and is affected by a number of factors including short-term plasticity [1-3], primarily involved in information filtering, long-term plasticity [4,5], involved in learning and memory [6], homeostatic plasticity [7], involved in the maintenance of function in the presence of changing environments, neuromodulation $[8,9]$, and astrocyte regulation $[10,11]$, in addition to the temporal properties of the presynaptic spikes, the intrinsic currents of the postsynaptic neurons and background noise activity.

Short-term plasticity (STP) (or short-term synaptic dynamics, STD) refers to the increase (synaptic facilitation) or decrease (synaptic depression) of the efficacy of synaptic transmission (strength of the synaptic conductance) over time in response to repeated presynaptic spikes with a duration in the range of milliseconds to minutes $[1-3,12,13]$. STP is ubiquitous both in invertebrate and vertebrate synapses, and has been shown to be important for neuronal computation [14-18] and information filtering (temporal and frequency-dependent) [12,19-38], and related phenomena such as burst detection [27,38], temporal coding and information processing [27, 28, 39-41], gain control [15, 42, 43], information flow [16, 36, 44] given the presynaptic history-dependent nature of STP, the prolongation of neural responses to transient inputs [45-47], the modulation of network responses to external inputs $[48,49]$, hearing and sound localization [50,51], direction selectivity [52], attractor dynamics [53] (see also [43]), navigation (e.g., place field sensing) [30,33], vision (e.g., microsacades) [54], working memory [47,55] and decision making [56].

The notion of information filtering as the result of synaptic depression or facilitation is associated with the concept of temporal filters at the synaptic and postsynaptic levels, which are better understood in response to periodic presynaptic inputs $[38,57,58]$ for a wide enough range of input frequencies. (See the schematic diagrams in Figs. 1-A and 2-A where $x$ and $z$ describe the evolution over time of synaptic depression and facilitation, respectively, and their product describes their combined activity assumed to be multiplicative.) In spite of the pervasiveness of STP and the consequences for information filtering, the mechanisms of generation of postsynaptic temporal filters in response to presynaptic input spikes are not well understood. In particular, it is not understood how the time scales associated with the dynamics of depression and facilitation interact with the presynaptic spike train time scale (interspike interval, ISI) and the postsynaptic membrane potential time scales to generate the resulting temporal filters, and how the dynamic properties of these temporal filters that govern their evolution over time depend on the said time scales.

Phenomenological models of synaptic depression and facilitation [21, 40,57-65] describe the two phenomena as the evolution of two variables that abruptly decrease or increase, respectively, by a certain amount in response to each presynaptic spike and relax towards their steady-state during the presynaptic ISIs in an independent manner (as in Figs. 1-A and 2-A). At the arrival of each presynaptic spike, the synaptic function $(S)$ is updated by an amount equal to the appropriate product of $x$ and $z$ (instead of a constant value as in equivalent models without STP).

The temporal filters (e.g., Figs. 1-A2), capturing the envelope of the depression and facilitation variables (e.g., Figs. 1-A1) develop as the result of the repetitive arrival of presynaptic spikes with rates of increase that depends on the value of the variables $x$ and $z$ at the arrival of each spike, which are controlled by both the time scales at which the relaxation of these variables to their steady-state occurs (e.g., the time constants $\tau_{d e p}$ and $\tau_{f a c}$ in Fig. 2-A1) and the presynaptic ISI.

These temporal filters can be captured by sequences of peak values $\left(X_{n}\right.$ and $Z_{n}$ where $n$ is the spike index) whose evolution are governed by difference equations and have time scales (e.g., $\sigma_{d e p}$ and $\sigma_{f a c}$ ) and steady states (e.g., $\bar{X}$ and $\bar{Z}$ ). We refer to them as a low-pass $\left(X_{n}\right)$ and high-pass $\left(Z_{n}\right)$ temporal filters, respectively. The synaptic update is the product $\Delta S_{n}=X_{n} Z_{n}$ and the corresponding filter can have a transient peak, which we refer to as a band-pass temporal filter and involve an additional time scale $\left(\sigma_{d e p+f a c}\right)$. These time scales depend on the single-event time scales $\left(\tau_{d e p}\right.$ and $\left.\tau_{f a c}\right)$ and the presynaptic ISI. In addition, the phenomenon of summation in a postsynaptic cell in response to presynaptic inputs develops an additional high-pass temporal filter (Fig. 2-B), which is independent of the ones described above, with a time scale $\left(\sigma_{\text {sum }}\right)$ and steady state $(\bar{S})$ that depend on the membrane time scale of the 
postsynaptic cell $\left(\tau_{m}\right)$, the presynaptic ISI and the synaptic decay time constant $\left(\tau_{d e c}\right)$.

In this paper we use biophysically plausible (conductance-based) mathematical modeling and dynamical systems tools to systematically understand how the postsynaptic low-, high- and band-pass temporal filters are generated in response to presynaptic spike trains in the presence of STP and how the dynamic properties of these filters depend on the interplay of the hierarchy of processes (ranging from the arrival of the presynaptic spike trains, to the activation of STP to the activation of the synaptic function to the response of the postsynaptic cell), and the hierarchy of temporal filters with their associated time scales, and in particular how all this depends on the time scales of the building blocks $\left(\tau_{d e p}, \tau_{f a c}, \tau_{d e c}, \tau_{m}\right.$ and the presynaptic ISI). The conceptual and mathematical framework we introduce to develop these ideas and identify the contribution of each of the components can be used to understand the filtering and coding properties of more complex scenarios involving more realistic description of the participating processes, non-periodic presynaptic spike trains (e.g., bursting patterns) and more complex networks.

\section{Methods}

\subsection{Models}

\subsubsection{Postsynaptic cell: leaky integrate-and-fire model}

The current-balance equation for the post-synaptic cell is given by

$$
C \frac{d V}{d t}=-g_{L}\left(V-E_{L}\right)+I_{a p p}-I_{\text {syn }}+I_{\text {noise }},
$$

where $t$ is time (ms), $V$ represents the voltage $(\mathrm{mV}), C$ is the specific capacitance $\left(\mu \mathrm{F} / \mathrm{cm}^{2}\right), g_{L}$ is the leak conductance $\left(\mathrm{mS} / \mathrm{cm}^{2}\right)$, lapp is the tonic $(\mathrm{DC})$ current $\left.\left(\mu \mathrm{A} / \mathrm{cm}^{2}\right)\right), I_{\text {noise }}=\sqrt{2 D} \eta(t)$ represents white noise (delta correlated with zero mean), and $I_{s y n}$ is an excitatory synaptic current of the form

$$
I_{s y n}=G_{e x} S\left(V-E_{e x}\right) .
$$

Here $G_{e x}$ is the maximal synaptic conductance $\left(\mathrm{mS} / \mathrm{cm}^{2}\right), E_{e x}=0$ is the reversal potential for AMPA excitation, and the synaptic variable $S$ obeys a kinetic equation of the form

$$
\frac{d S}{d t}=-\frac{S}{\tau_{d e c}}+\Delta S_{n} \delta\left(t-t_{s p k}\right)
$$

where $\tau_{\text {dec }}(\mathrm{ms})$ is the decay time of excitation. Each presynaptic spike instantaneously raises $S$ to some value $\Delta S_{n}$ which varies depending on the properties of the short-term dynamics (depression and/or facilitation) and defined below. We refer the reader to $[59,66]$ for additional details on biophysical (conductance-based) models.

\subsubsection{Presynaptic spike-trains}

We model the spiking activity of the presynaptic cell as a spike train with presynaptic spike times $t_{1}, t_{2}, \ldots, t_{N}$. We consider two types of input spike-trains: uniformly and Poisson distributed. The former is characterized by the interspike interval (ISI) of length $\Delta_{s p k}$ (or its reciprocal, the spiking frequency $f_{s p k}$ ) and the latter are characterized by the mean spiking rate (or the associated exponential distribution of ISIs).

\subsubsection{DA phenomenological model for short-term dynamics: synaptic depression and facilitation}

This simplified phenomenological model has been introduced in [59]. It is relatively simpler than the wellknown phenomenological MT model described below [58]. In particular, the depression and facilitation 
processes are independent (the updates upon arrival of each presynaptic spike are uncoupled). We use it for its tractability and to introduce some conceptual ideas.

The magnitude $\Delta S$ of the synaptic release per presynaptic spike is assumed to be the product of the depressing and facilitating variables

$$
\Delta S=x^{-} z^{+}
$$

where

$$
\frac{d x}{d t}=\frac{x_{\infty}-x}{\tau_{d e p}}-a_{d} x \delta\left(t-t_{s p k}\right),
$$

and

$$
\frac{d z}{d t}=\frac{z_{\infty}-z}{\tau_{f a c}}+a_{f}(1-z) \delta\left(t-t_{s p k}\right) .
$$

Each time a presynaptic spike arrives $\left(t=t_{s p k}\right)$, the depressing variable $x$ is decreased by an amount $a_{d} x$ (the release probability is reduced) and the facilitating variable $z$ is increased by an amount $a_{f}(1-z)$ (the release probability is augmented). During the presynaptic interspike intervals (ISIs) both $x$ and $z$ decay exponentially to their saturation values $x_{\infty}$ and $z_{\infty}$ respectively. The rate at which this occurs is controlled by the parameters $\tau_{d e p}$ and $\tau_{f a c}$. Following others we use $x_{\infty}=1$ and $z_{\infty}=0$. The superscripts " \pm " in the variables $x$ and $z$ indicate that the update is carried out by taking the values of these variables prior $\left(_{-}\right)$or after $\left(^{+}\right.$the arrival of the presynaptic spike.

Fig. 1-A1 illustrates the $x$-, $z$ - and $M=x z$-traces (defining the $\Delta S$-traces) (curves of $x, z$ and $M$ as a function of time) in response to a periodic presynaptic input train for representative parameter values. (Note that $M$ is a continuous variable, while $\Delta S$ is used for the update after the arrival of spikes, and $\Delta S=R^{-} u^{+}$and $\Delta S_{n}$ is the sequence off peaks.)

\subsubsection{DA model in response to presynaptic inputs}

\section{Peak dynamics and temporal filters}

By solving the differential equations (5)-(6) during the presynaptic ISIs and appropriately updating the solutions at $t=t_{n}$ (occurrence of each presynaptic spike), one arrives at the following recurrent formula for the peak sequences in terms of the model parameters

$$
X_{n+1}=x_{\infty}+\left[\left(1-a_{d}\right) X_{n}-x_{\infty}\right] e^{-\Delta_{s p k, n} / \tau_{d e p}}
$$

and

$$
Z_{n+1}=a_{f}+\left(1-a_{f}\right)\left[z_{\infty}+\left(Z_{n}-z_{\infty}\right) e^{-\Delta_{s p k, n} / \tau_{f a c}}\right]
$$

where $\left\{\Delta_{s p k, n}\right\}_{n=1}^{N s p k}$ represents the lengths of the presynaptic ISIs.

Fig. 1-A2 illustrates the peak envelopes (curves joining the peak sequences for $X_{n}, Z_{n}$ and $\Delta S_{n}=$ $X_{n} Z_{n}$, circles) for the parameter values in Fig. 1-A1. These are sequences indexed by the input spike number, which we calculate analytically below. As expected, $X_{n}$ is a decreasing sequence (temporal low-pass filter) and $Z_{n}$ is an increasing sequence (temporal high-pass filter). Their product (computed so that the peak of the product is the product of the peaks) exhibits a transient peak (temporal band-pass filter). 
A1

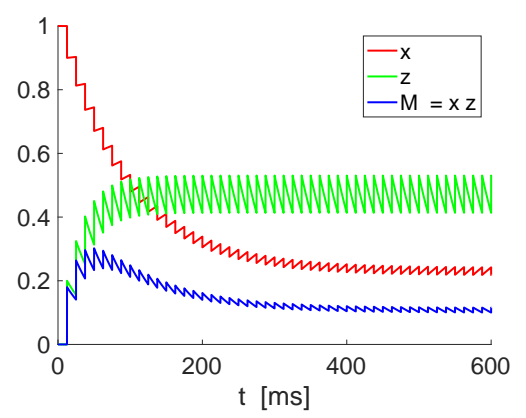

B1

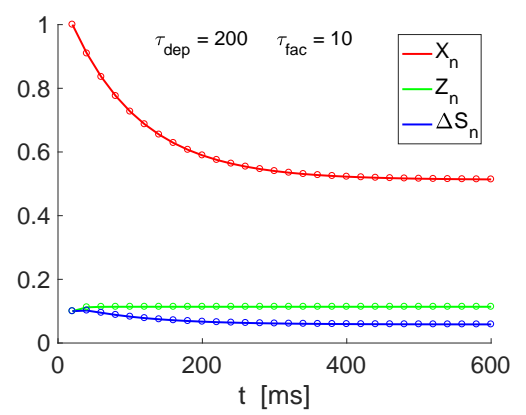

C1

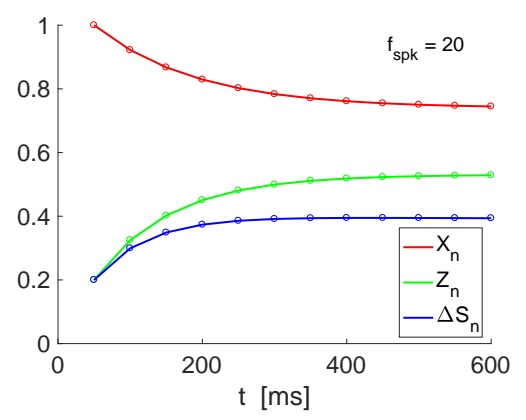

A2

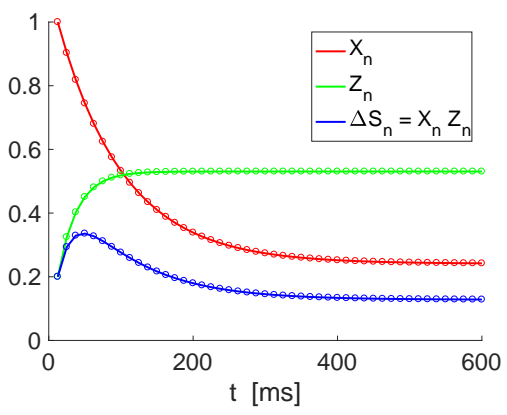

B2

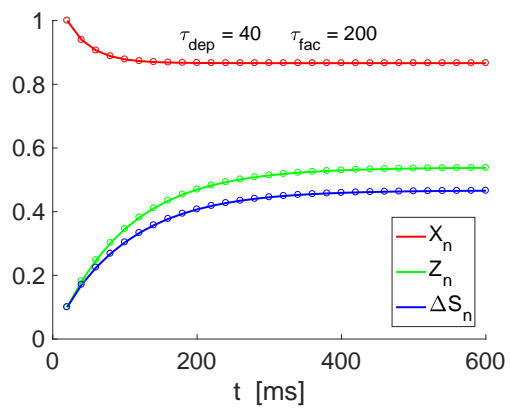

C2

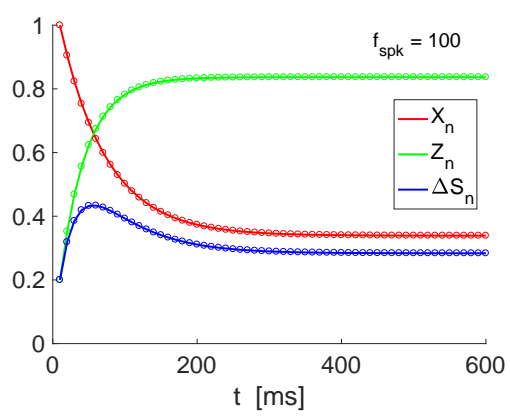

Figure 1: Short-term depression and facilitation and the generation of temporal filters in response to periodic presynaptic inputs. A1. $x-, z$ - and $M$-traces (curves of $x, z$ and $M$ as a function of $t$ ). A2. Circles: $X_{n^{-}}, Z_{n}$ - and $\Delta S_{n}=X_{n} Z_{n}$ - peak sequence computed using (7)-(8). Solid curves: join the $X_{n}-, Z_{n}$ - and $\Delta S_{n}=X_{n} Z_{n}$ - envelope peak sequences computed using the caricature (descriptive) model (32)-(35). The values of the envelope peaks decay constants are $\sigma_{d} \sim 91.5$ and $\sigma_{f} \sim 26.4$. We used the simplified model (4)-(6) and the following parameter values: $\tau_{d e p}=400, \tau_{f a c}=50, a_{d}=0.1, a_{f}=0.2, x_{\infty}=1$, $z_{\infty}=0, f_{s p k}=80 \mathrm{~Hz}$ (presynaptic input frequency). B. Depression- and facilitation-dominated peak sequences. B1. Depressiondominated temporal filter regime. B2. Facilitation-dominated temporal filter regime. We used the simplified model (4)-(6) and the following parameter values: $\tau_{d e p}=200$ (B1), $\tau_{d e p}=40$ (B2), $\tau_{f a c}=10$ (B1), $\tau_{f a c}=200$ (B2), $a_{d}=0.1, a_{f}=0.1$, $x_{\infty}=1, z_{\infty}=0, f_{s p k}=50 \mathrm{~Hz}$. C. Input frequency-dependent temporal filters. C1. High-pass temporal filter for low spiking input frequencies $\left(f_{s p k}=20\right)$. C2. Band-pass temporal filter for higher spiking input frequencies $\left(f_{s p k}=100\right)$. We used the simplified model (4)-(6) and the following parameter values: $\tau_{d e p}=200, \tau_{f a c}=200, a_{d}=0.1, a_{f}=0.2, x_{\infty}=1, z_{\infty}=0$. 
A1

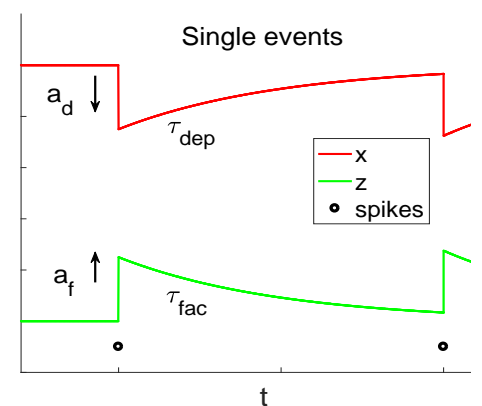

B1

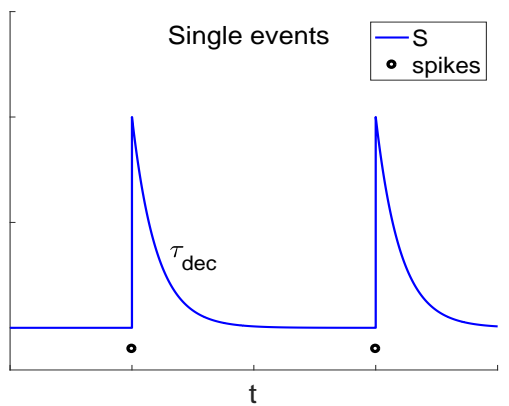

A2

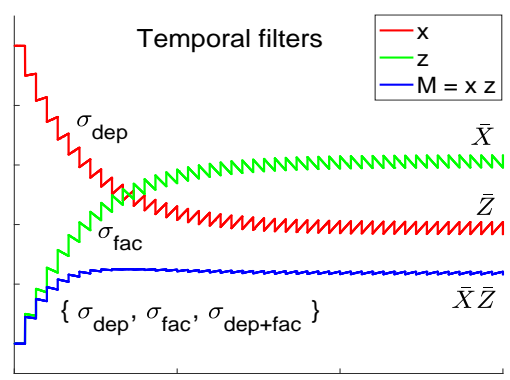

$\mathrm{t}$

B2

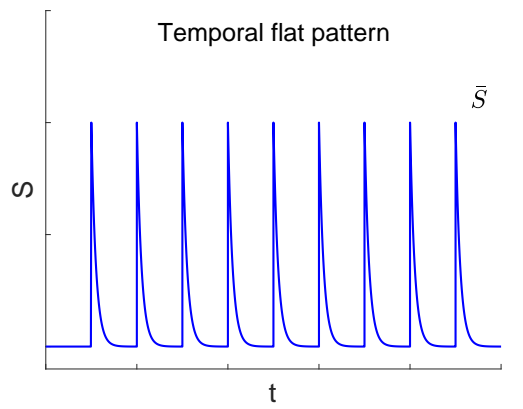

A3

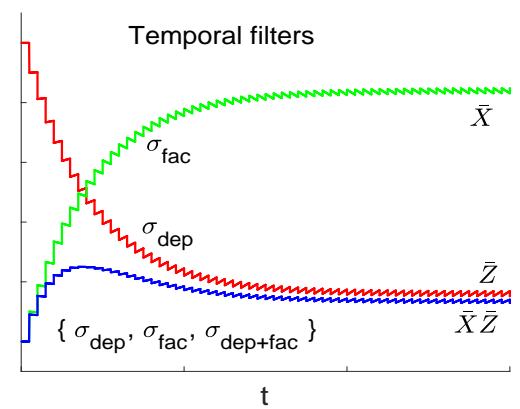

B3

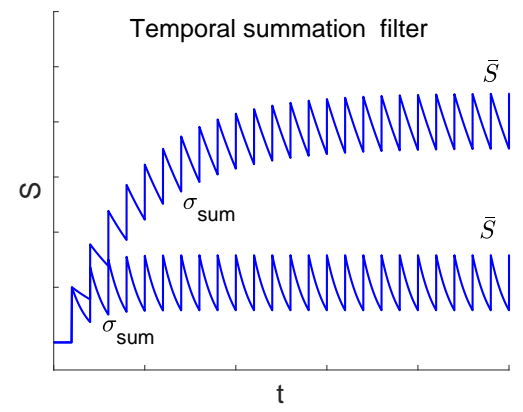

Figure 2: Single event and temporal filters' time scales and other attributes in response to presynaptic spike trains in the presence of synaptic depression $(x)$ and facilitation $(z)$. A. Depression and facilitation. A1. Single events. At the arrival of each presynaptic spike (black dots), the depression $(x)$ and facilitation $(z)$ variables decrease and increase, respectively. In the models we use in this paper, they are discretely updated. They decay towards their (single event) steady-states $\left(x_{\infty}=1\right.$ and $\left.z_{\infty}=0\right)$ with the (single event) time scales $\tau_{d e p}$ and $\tau_{f a c}$, respectively. A2, A3. Temporal patterns (filters) generated by presynaptic spike trains with different ISIs $\Delta_{s p k}$ (or frequencies $f_{s p k}$ ) and the dynamics of the single events (controlled by $\tau_{d e p}$ and $\tau_{f a c}$ ). Depression and facilitation always give rise to low- (red) and high- (green) pass filters respectively. Their product can be a depression-dominated (low-pass) filter, facilitation-dominated (high-pass) filter (A2), or a band-pass filter (A3). The (emergent, long term) filter time scales $\sigma_{d e p}, \sigma_{f a c}$ and $\sigma_{d e p+f a c}$ depend on the interplay of $\tau_{d e p}, \tau_{f a c}$ and $\Delta_{s p k}$. The temporal filter steady-states are captured by the peak sequence steady-states $\bar{X}, \bar{Z}$ and $\bar{\Delta} S=\bar{X} \bar{Z}$. B. Synaptic dynamics. B1. Single events. At the arrival of each presynaptic spike (black dots) the synaptic variable $X$ increases and then decreases with a time constant $\tau_{d e c}$, which defines the decay time scale. In the models we use in this paper, $S$ is discretely updated. B2, B3. Temporal patterns (filters) generated by presynaptic spike trains with different ISIs $\Delta_{s p k}$ (or frequencies $f_{s p k}$ ) and the dynamics of the single events (controlled by $\tau_{d e c}$ ). For small $\tau_{d e c}$ and $\Delta_{s p k}$, the $S$ pattern is flat (B2). For larger values of $\tau_{d e c}$ and $\Delta_{s p k}$, summation generates a high-pass filter. The emergent time scales depend on the interplay of $\tau_{d e c}$ and $\Delta_{s p k}$. The temporal filter steady-states are captured by the peak sequence steady-states $\bar{S}$. 


\section{Steady-state frequency-dependent filters}

For periodic inputs, $\Delta_{s p k, n}$ is independent of $n\left(\Delta_{s p k}\right)$ and eqs. (7)-(8) are linear 1D difference equations. Therefore both the sequences $X$ and $Z$ obey linear discrete dynamics (e.g., see [67]), decaying to their steady state values

$$
\bar{X}=\frac{\left(1-e^{-\Delta_{s p k} / \tau_{d e p}}\right) x_{\infty}}{1-\left(1-a_{d}\right) e^{-\Delta_{s p k} / \tau_{d e p}}}
$$

and

$$
\bar{Z}=\frac{\left(1-e^{-\Delta_{s p k} / \tau_{f a c}}\right)\left(1-a_{f}\right) z_{\infty}+a_{f}}{1-\left(1-a_{f}\right) e^{-\Delta_{s p k} / \tau_{f a c}}}
$$

as shown in Figs. 1 -B and $-\mathrm{C}$ (red and green).

\subsubsection{MT phenomenological model for short-term dynamics: synaptic depression and facilitation}

This model was introduced in in [58] as a simplification of earlier models $[1,40,68]$. It is more complex and more widely used than the DA model described above $[38,69]$, but still a phenomenological model.

As for the DA model, the magnitude $\Delta S$ of the synaptic release per presynaptic spike is assumed to be the product of the depressing and facilitating variables:

$$
\Delta S=R^{-} u^{+}
$$

where, in its more general formulation,

$$
\frac{d R}{d t}=\frac{1-R}{\tau_{d e p}}-R^{-} u^{+} \delta\left(t-t_{s p k}\right),
$$

and

$$
\frac{d u}{d t}=\frac{\hat{U}-u}{\tau_{f a c}}+U\left(1-u^{-}\right) \delta\left(t-t_{s p k}\right) .
$$

Each time a presynaptic spike arrives $\left(t=t_{s p k}\right)$, the depressing variable $R$ is decreased by $R^{-} u^{+}$and the facilitating variable $u$ is increased by $U\left(1-u^{-}\right)$. As before, the superscripts " \pm " in the variables $R$ and $u$ indicate that the update is carried out by taking the values of these variables prior $\left(R^{-}, u^{-}\right)$or after $\left(R^{+}, u^{+}\right)$the arrival of the presynaptic spike. In contrast to the DA model, the update of the depression variable $R$ is affected by the value of the facilitation variable $u^{+}$.

Simplified versions of this model include making $\bar{U}=0[21,57,58,63,65,70]$ and $\bar{U}=U[38]$ where the updates do not discriminate between the values of the participating variables before or after the arrival of spikes

\subsubsection{MT model in response to presynaptic inputs}

\section{Peak dynamics and temporal filters}

By solving the differential equations (12)-(13) during the presynaptic ISIs and appropriately updating the solutions at $t=t_{n}$ (occurrence of each presynaptic spike), one arrives at the following recurrent formula for the peak sequences in terms of the model parameters

$$
R_{n+1}=R_{n}\left(1-u_{n+1}\right) e^{-\Delta_{s p k} / \tau_{d e p}}+1-e^{-\Delta_{s p k, n} / \tau_{d e p}}
$$


and

$$
u_{n+1}=\hat{U}+U-\hat{U} U+u_{n}(1-U) e^{-\Delta_{s p k, n} / \tau_{f a c}}-\hat{U}(1-U) e^{-\Delta_{s p k, n} / \tau_{f a c}}
$$

\section{Steady-state frequency-dependent filters}

As before, for presynaptic inputs $\Delta_{s p k, n}$ is independent of $n$ and these equations represent a system two 1D difference equations, which are now nonlinear. The steady-state values are given by

$$
\bar{R}=\frac{1-e^{-\Delta_{s p k} / \tau_{d e p}}}{1-(1-\bar{u}) e^{-\Delta_{s p k} / \tau_{d e p}}}
$$

and

$$
\bar{u}=\frac{\hat{U}+U-\hat{U} U-\hat{U}(1-U) e^{-\Delta_{s p k} / \tau_{f a c}}}{1-(1-U) e^{-\Delta_{s p k} / \tau_{f a c}}}
$$

\subsubsection{Synaptic dynamics in response to periodic presynaptic inputs and a constant up- date}

\section{Peak dynamics}

By solving the differential equation (3) for a constant value of $\Delta S_{n}=\Delta S$ during the presynaptic ISIs and updating the solution at each occurrence of the presynaptic spikes at $t=t_{n}, n=1, \ldots, N_{s p k}$, one arrives to the following discrete linear differential equation for the peak sequences in terms of the model parameters

$$
S_{n+1}=e^{-\Delta_{s p k} / \tau_{d e c}} S_{n}+\Delta S
$$

\section{Steady-states and frequency filters}

The steady state values of (18) are given by

$$
\bar{S}=\frac{\Delta S}{1-e^{-\Delta_{s p k} / \tau_{d e c}}} .
$$

\subsection{Numerical simulation}

The numerical solutions were computed using the modified Euler method (Runge-Kutta, order 2) [71] with a time step $\Delta t=0.01 \mathrm{~ms}$ (or smaller values of $\Delta t$ when necessary) in MATLAB (The Mathworks, Natick, MA).

\section{Results}

\subsection{Temporal summation filters: the single-event and the (long-term) filter time scales coincide for constant updates}

Temporal summation filters (SFs, Fig. 2-B3) refer to the long-term patterns generated in the response of a dynamical system to periodic stimulation by the accumulation of activity in response to the single events 
(responses to each stimulation event, local in time). Temporal summation synaptic filters are HPFs and naturally develop in the response of linear systems such as eq. (18) with a constant update (independent of the spike index) where the activity $S$ decays during the presynaptic ISI and it is updated in an additive manner at the arrival of each presynaptic spike. If the quotient $\Delta_{s p k} / \tau_{d e c}$ is finite, $S$ will not be able to reach a small enough vicinity of zero before the next presynaptic spike arrives and then the $S$-peak envelope will increase across cycles. While summation does not require the presynaptic inputs to be periodic, the notion of filter we use here does.

The solution to equation (18) is given by

$$
S_{n}=\bar{S}+e^{-(n-1) \Delta_{s p k} / \tau_{d e c}}\left(S_{1}-\bar{S}\right),
$$

where $S_{1}=\Delta S$ (see Appendix A). This equation describes the temporal filter in response to the presynaptic spike train. For technical purposes, one can extend eq. (20) to include the point $(0,0)$, obtaining

$$
S_{n}=\bar{S}\left(1-e^{-n \Delta_{s p k} / \tau_{d e c}}\right) .
$$

A further extension to the continuous domain yield

$$
S_{t}=\bar{S}\left(1-e^{-t / \tau_{d e c}}\right) .
$$

which is the solution to

$$
\tau_{d e c} \frac{d S_{t}}{d t}=\bar{S}-S_{t}
$$

We use the notation $S_{t}$ instead of $S(t)$ to emphasize the origin of $S_{t}$ as the continuous extension of a discrete sequence rather than the evolution of Eq. (3).

Together these results show that the time scale $\tau_{d e c}$ for the single events (in response to each presynaptic spike is directly communicated to the temporal SF in a $\Delta_{s p k}$-independent manner. In other words, the dynamics of both the temporal SF and the single events are controlled by the same time constant $\tau_{d e c}$. While the time scale is independent of $\Delta_{s p k}$, the steady-state $\bar{S}$ is $\Delta_{s p k}$-dependent.

\subsection{Dynamics of the depression $(x)$ and facilitation $(z)$ variables and their inter- action: emergence of temporal low-, high-and band-pass filters}

\subsubsection{From single events (local in time) to temporal patterns and filters (global in time)}

The dynamics of single events for the variables $x$ (depression) and $z$ (facilitation) are governed by eqs. (5)-(6) , respectively. After the update upon the arrival of a spike, $x$ and $z$ decay towards their saturation values $x_{\infty}(=1)$ and $z_{\infty}(=0)$, respectively.

The response of $x$ and $z$ to repetitive input spiking generates patterns for these variables in the temporal domain (e.g., Fig. 1-A1) and for the peak sequences $X_{n}$ and $Z_{n}$ in the (discrete) presynaptic spike-time domain (e.g., Fig. 1-A2). They consist of their transition from the initial peaks $X_{1}$ and $Z_{1}$ to $\bar{X}$ and $\bar{Z}$, respectively, as $n \rightarrow \infty$. The properties of these patterns depend not only on the parameters for the single events $\left(\tau_{d e p / f a c}\right.$ and $a_{d / f}$, but also on the input frerquency $f_{s p k}$ (or the presynaptic ISI $\left.\Delta_{s p k}\right)$ as reflected by eqs. (9)-(10) describing the peak-envelope steady-state values.

The peak envelope patterns have emergent, long-term time constants, for which we use the notation $\sigma_{d}$ and $\sigma_{f}$ (see also Section 3.3.2), which depend on $\tau_{d e p / f a c}, \Delta_{s p k}$ and $a_{d / f}$. The relationship between the long- and (single event) short-term time scales is more complex than straightforward identity between the two time scales discussed in the previous section where the single events were controlled only by the time constant. We discuss this in more detail later in the next section.

The $\Delta S_{n}$ envelope patterns combine these time scales in ways that involve different levels of complexity. $\Delta S_{n}$ patterns like the one shown in Fig. 1-A (blue) that exhibit a peak in the temporal domain 
(transient peak) have been referred to in the literature as temporal filters, borrowing the language from electric circuits in the frequency domain that exhibits resonance (including neuronal circuits). Here we refer to this type of patterns as temporal band-pass filters (BPFs) and to the $\Delta S_{n}$ patterns that are monotonically decreasing (Fig. 1-A, red) and increasing (Fig. 1-A, green) as temporal low- and high-pass filters (LPF and HPF), respectively.

\subsubsection{Depression- / facilitation-dominated regimes and transitions between them}

In the absence of either facilitation or depression, $\Delta S_{n}$ temporal LPFs and HPFs reflect the presence of depressing or facilitating synapses, respectively. However, $\Delta S_{n}$ temporal LPFs and HPFs need not be generated by pure depression and facilitation but can reflect different balances between these processes where either depression (Fig. 1-B1) or facilitation (Fig. 1-B2) dominates.

It is instructive to look at the limiting cases. A small enough value of $\tau_{d e p / f a c}$ causes a fast recovery to the saturation value $\left(x_{\infty}\right.$ or $\left.z_{\infty}\right)$ and therefore the corresponding sequence $\left(X_{n}\right.$ or $\left.Z_{n}\right)$ is almost constant. In contrast, a large enough value of $\tau_{d e p / f a c}$ causes a slow recovery to the saturation value and therefore the corresponding sequence shows a significant decrease $\left(X_{n}\right)$ or increase $\left(Z_{n}\right)$ as the result of the corresponding underlying variables $(x$ and $z)$, being almost constant during the ISI. Therefore, when $\tau_{d e p} \gg \tau_{f a c}$, depression dominates (Fig. 1-B1) and when $\tau_{d e p} \ll \tau_{f a c}$, facilitation dominates (Fig. 1-B2).

In both regimes, the exact ranges depend on the input frequency $f_{s p k}$ (or the ISI $\Delta_{s p k}$ ). An increase in $f_{s p k}$ (decrease in $\Delta_{s p k}$ ) reduces the ability of $x$ and $z$ to recover to their saturation values within each presynaptic ISI, and therefore amplifies the depression and facilitation effects over the same time interval and over the same amount of input spikes (compare Figs. 1-C1 and -C2). Therefore, the different balances between $X_{n}$ and $Z_{n}$ as $f_{s p k}$ generate different types of $\Delta S_{n}$ patterns and may cause transitions between qualitatively different $\Delta S_{n}$ patterns.

\subsection{Dependence of the (depression/facilitation) temporal low- and high-pass fil- ter time scales on the single event time scales}

\subsubsection{Communication of the single event time scales to the (long-term) history-dependent filters}

Here we focus on understanding how the long-term time scale of the depressing and facilitating sequences $X_{n}$ and $Z_{n}$ (peak-envelope response to periodic presynaptic spikes) result from the interaction of the time constants for the single events $\left(\tau_{d e p}\right.$ and $\left.\tau_{f a c}\right)$ and the spiking input time scales $\Delta_{s p k}$.

From eqs. (7)-(8), which govern the evolution of the temporal sequences $X_{n}$ and $Z_{n}$, their dynamics depend on the recovery time constants for the single events $\left(\tau_{d e p}\right.$ or $\left.\tau_{f a c}\right)$, the ISI of the presynaptic spikes $\Delta_{s p k}$ and the update parameters $\left(a_{d}\right.$ and $a_{f}$, also for the single events). Standard methods (see Appendix A) applied to these linear difference equations for $\Delta_{s p k, n}=\Delta_{s p k}$ (independent of $n$ ) yield

$$
X_{n}=\bar{X}+Q\left(a_{d}, \tau_{d e p}\right)^{n-1}\left(X_{1}-\bar{X}\right)
$$

and

$$
Z_{n}=\bar{Z}+Q\left(a_{f}, \tau_{f a c}\right)^{n-1}\left(Z_{1}-\bar{Z}\right)
$$

for $n=1, \ldots, N_{s p k}$, where

$$
Q\left(a_{s t p}, \tau_{s t p}\right)=\left(1-a_{s t p}\right) e^{-\Delta_{s p k} / \tau_{s t p}}
$$

and

$$
f_{s p k}=1000 / \Delta_{s p k} .
$$


The temporal patterns for the peak sequences $X_{n}$ and $Z_{n}$ (their transition from $X_{1}$ and $Z_{1}$ to $\bar{X}$ and $\bar{Z}$, respectively, as $n \rightarrow \infty)$ are spanned as the factors $Q\left(a_{d}, \tau_{d e p}\right)^{n-1}$ and $Q\left(a_{f}, \tau_{f a c}\right)^{n-1}$ approach zero (e.g., Figs. 3, gray). Because this occurs monotonically (in both cases), then the $\left\{X_{n}\right\}_{n=1}^{\infty}$ and $\left\{Z_{n}\right\}_{n=1}^{\infty}$ patterns decrease and increase monotonically to $\bar{X}$ and $\bar{Z}$, respectively (e.g., Figs. 1 and 3 , red and green dots). The convergence for $a_{\text {stp }}<1$ is guaranteed by the fact that $Q\left(a_{\text {stp }}, \tau_{\text {stp }}\right)<1$. Biophysically plausible values of $a_{d}$ and $a_{f}$ are well within this range.

A1

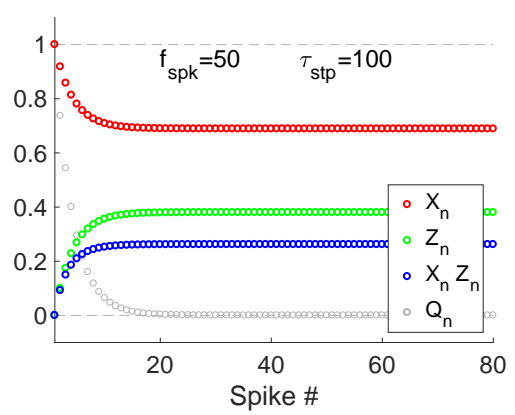

B1

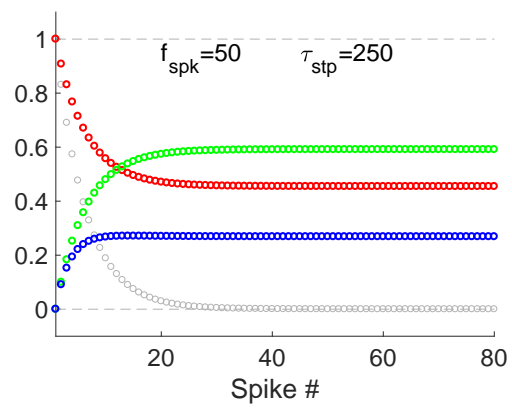

C1

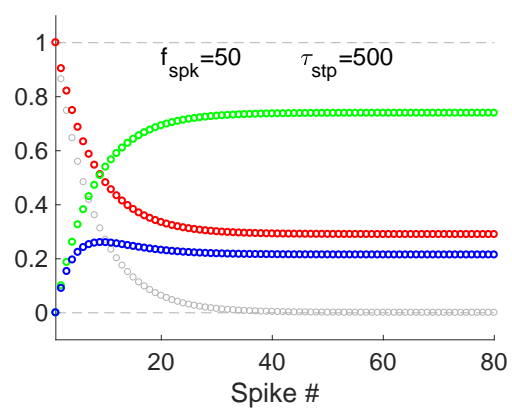

A2

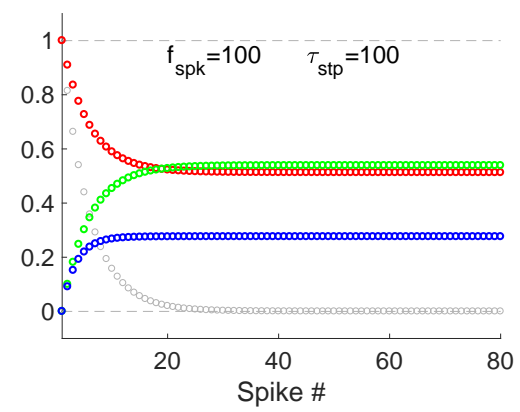

B2

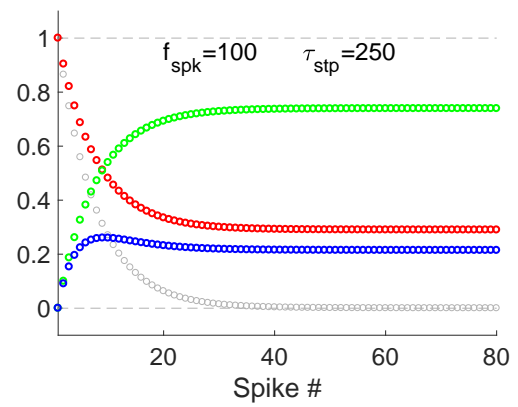

C2

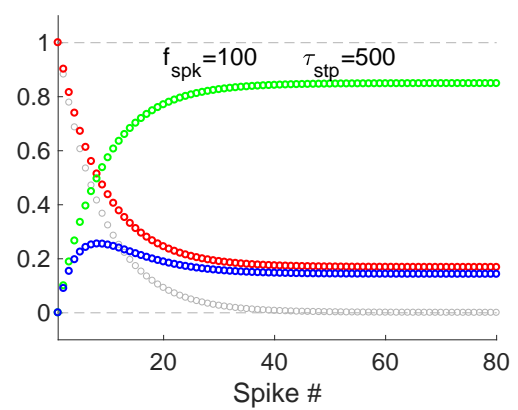

A3

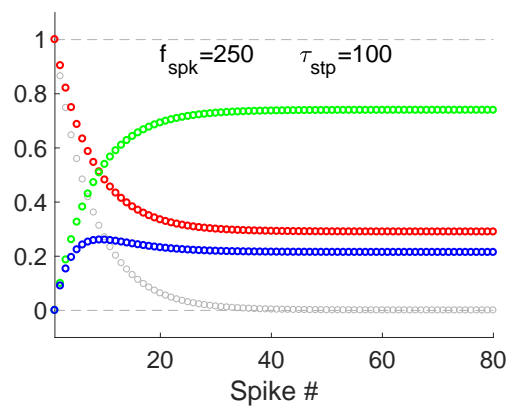

B3

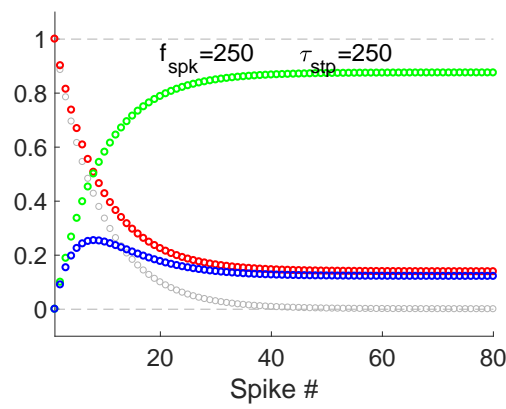

C3

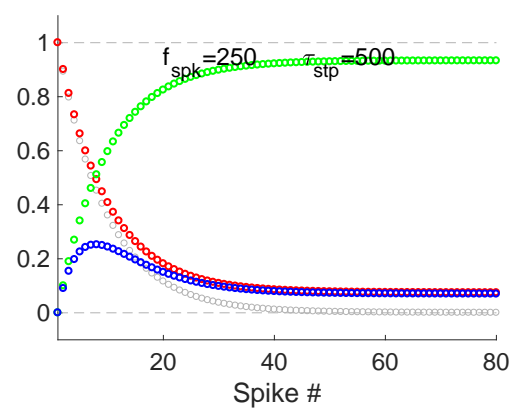

Figure 3: Low-, high- and band-pass filters in response to periodic presynaptic inputs in the presence of synaptic depression and facilitation: peak envelope dynamics. The evolution of the peak sequences $X_{n}$ (depression, red) and $Z_{n}$ (facilitation, green), respectively are governed by eqs. (24)-(26) and the sequence $Q_{n}=Q\left(a_{s t p}, \tau_{s t p}\right)^{n-1}$ (light gray) is given by eq. (26). We used the same parameter values for depression and facilitation: $\tau_{d e p}=\tau_{f a c}=\tau_{s t p}$ and $a_{d}=a_{f}=a_{s t p}=0.1$. A. $\tau_{s t p}=100$. A1. $f_{s p k}=50$. A2. $f_{s p k}=100$. A3. $f_{s p k}=250$. B. $\tau_{s t p}=250$. B1. $f_{s p k}=50$. B2. $f_{s p k}=100$. B3. $f_{s p k}=250$. C. $\tau_{s t p}=500$. C1. $f_{s p k}=50$. C2. $f_{s p k}=100$. C3. $f_{s p k}=250$. We used the folowing additional parameter values: $x_{\infty}=1$ and $z_{\infty}=0$. 
For each value of $n, Q^{n-1}$ is an increasing function of $Q$ and for each fixed value of $Q, Q^{n-1}$ is a decreasing function of $n$. Together, the larger $Q\left(a_{s t p}, \tau_{s t p}\right)$, the larger the sequence $Q_{n}=Q\left(a_{s t p}, \tau_{s t p}\right)^{n-1}$ and the slower $Q_{n}=Q\left(a_{s t p}, \tau_{s t p}\right)^{n-1}$ converges to zero. From eq. (26), all other parameters fixed, $Q\left(a_{s t p}, \tau_{s t p}\right)^{n-1}$ decreases slower the smaller $\Delta_{s p k}$ (larger $\left.f_{s p k}\right)$ (compare Fig. 4 columns 1 to 3 ), the larger $\tau_{s t p}$ (compare Fig. 4 rows 1 to 3 ) and the smaller $a_{s t p}$ (not shown in the figure).

A1

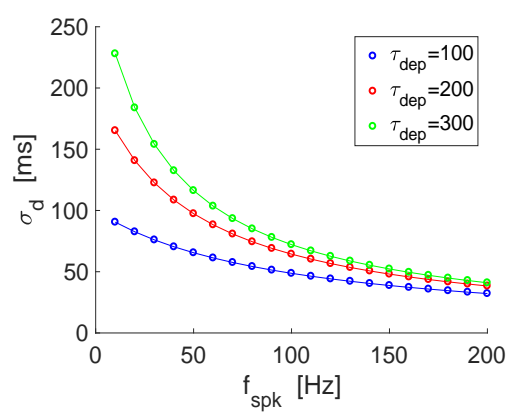

B1

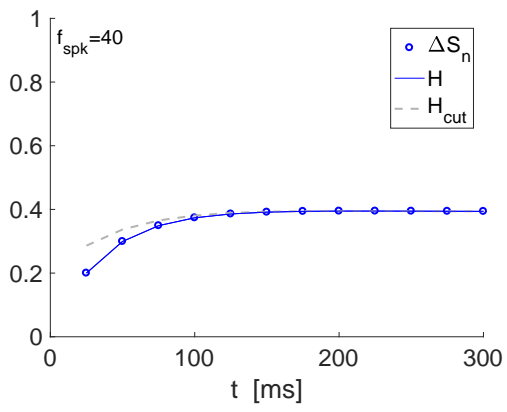

B4

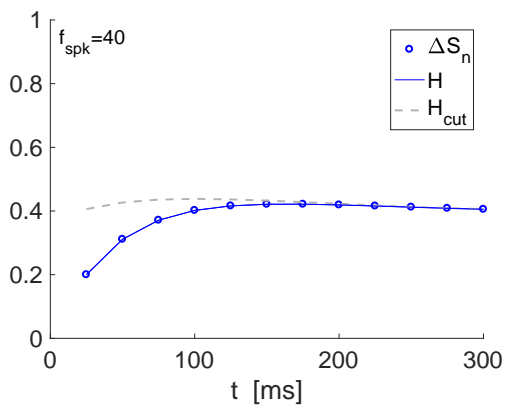

A2

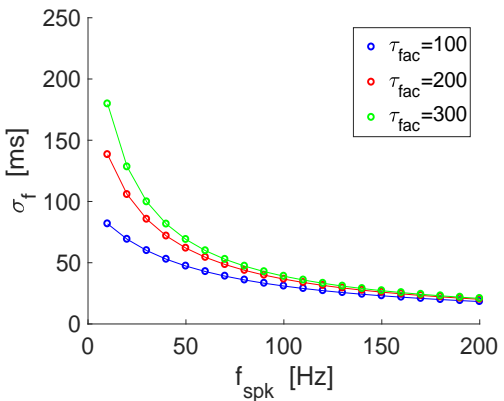

B2

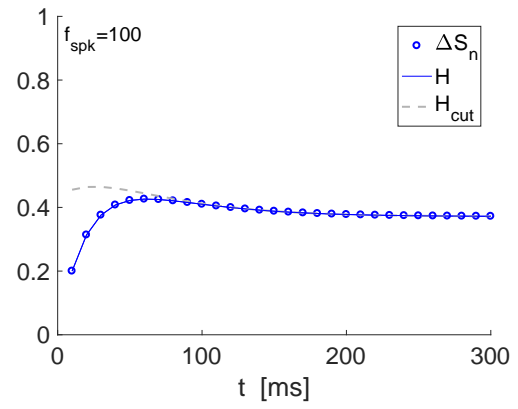

B5

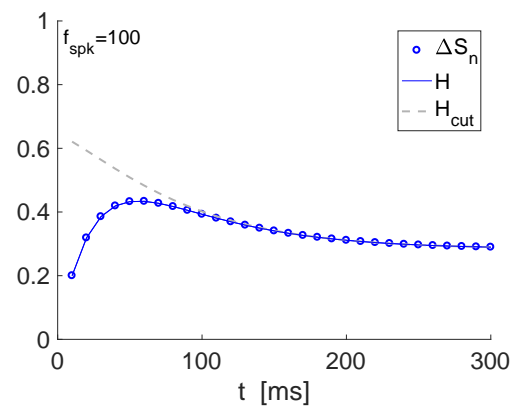

A3

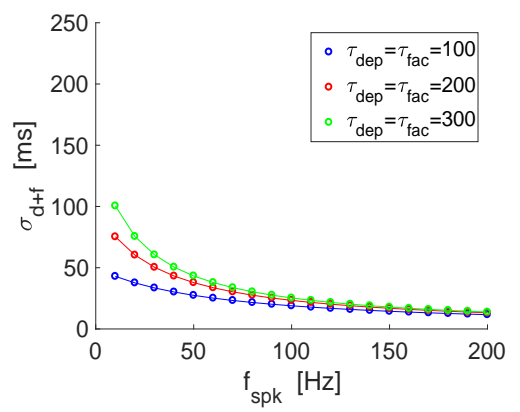

B3

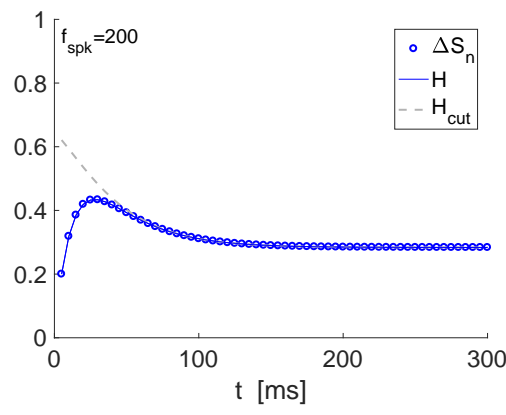

B6

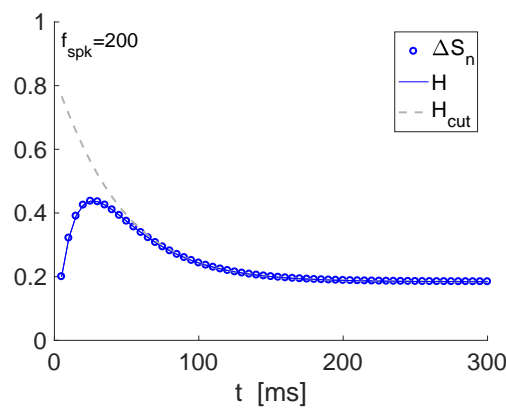

Figure 4: The time scales for the peak envelope responses $X_{n}$ and $Z_{n}$ to periodic presynaptic spikes and $\Delta S_{n}$ temporal filters $\left(\sigma_{d}, \sigma_{f}\right.$ and $\left.\sigma_{d+f}\right)$ depend on the interplay of $\tau_{d e p}, \tau_{f a c}$ and $f_{s p k}$ (or $\Delta_{s p k}$ ). A. Dependence of $\sigma_{d}, \sigma_{f}$ and $\sigma_{d+f}$ with the presynaptic input frequency $f_{s p k}$. A1. $\sigma_{d}$ for $a_{d e p}=0.1$. A2. $\sigma_{f}$ for $a_{f a c}=0.2$. A3. $\sigma_{d+f}$ computed using eq. (44) from $\sigma_{d}$ and $\sigma_{f}$ in panels $\mathrm{A} 1$ and A2. B. The contribution of the combined time scale $\sigma_{d+f}$ increases with $f_{s p k}$. The function $H\left(t_{k}\right)$ is given by (43) and the function $H_{c u t}$ consists of the three first terms in (43). B1-B3. $\tau_{d e p}=\tau_{f a c}=100$. B4-B6. $\tau_{d e p}=\tau_{f a c}=200$. We used the simplified model (4)-(6) and the formulas (35) for the (simplified) descriptive model, together with (7)-(10) and the following parameter values: $a_{d}=0.1, a_{f}=0.2, x_{\infty}=1, z_{\infty}=0$.

The effective time scale of the sequence $Q_{n}$ can be quantified by calculating the time it takes for $Q_{n}$ 
to decrease from $Q_{1}=1$ to 0.37 and multiply this number by $\Delta_{s p k}$. This yields

$$
\sigma_{Q}=\frac{\ln (0.37) \Delta_{s p k}}{\ln \left(1-a_{s t p}\right)-\Delta_{s p k} / \tau_{s t p}} \sim \frac{1}{1 / \tau_{s t p}-\ln \left(1-a_{s t p}\right) / \Delta_{s p k}},
$$

where $\sigma_{Q}$ is expressed in decimal numbers and has units of time. By substituting $\tau_{s t p}$ and $a_{s t p}$ by $\tau_{d e p}$ and $a_{d}\left(X_{n}\right)$ and by $\tau_{f a c}$ and $a_{f}\left(Z_{n}\right)$, respectively, we obtain the time scales $\sigma_{d e p}$ and $\sigma_{f a c}$ for the sequences $X_{n}$ and $Z_{n}$. These time scales quantify the time it takes for $X_{n}$ to decrease from $X_{1}$ to $X_{1}-0.63\left(X_{1}-\bar{X}\right)$ and for $Z_{n}$ to increase from $Z_{1}$ to $Z_{1}+0.63\left(\bar{Z}-Z_{1}\right)$, respectively.

While the dynamics of $Q\left(a_{s t p}, \tau_{s t p}\right), X_{n}$ and $Z_{n}$ depend on the quotient $\Delta_{s p k} / \tau_{s t p}$ (the two interacting time scales for the single events), the long-term time scales $\left(\sigma_{Q}, \sigma_{d}\right.$ and $\left.\sigma_{f}\right)$ depend on these quantities in a more complex way (Fig.5-A). For the limiting case $f_{s p k} \rightarrow 0, \sigma_{d e p / f a c} \rightarrow \tau_{d e p / f a c}$. For the limiting case $f_{s p k} \rightarrow \infty, \sigma_{d e p / f a c} \rightarrow 0$. For values of $f_{s p k}$ in between (see Appendix B), $\sigma_{d e p}$ and $\sigma_{f a c}$ decrease between these extreme values $\left(d \sigma_{d e p / f a c} / d \Delta_{s p k}<0\right)$. The dependence of $\sigma_{d e p}$ and $\sigma_{f a c}$ with $\tau_{d e p}$ and $\tau_{f a c}$ follows a different pattern since $\left(d \sigma_{d e p / f a c} / d \tau_{d e p / f a c}>0\right)$. Both $\sigma_{d e p}$ and $\sigma_{f a c}$ increase with $\tau_{d e p}$ and $\tau_{f a c}$, respectively. The dependence of $\sigma_{d e p}$ and $\sigma_{f a c}$ with $a_{d}$ and $a_{f}$ follows a similar pattern $\left(d \sigma_{d / f} / d a_{d / f}>0\right)$. Details for these calculations are presented in the Appendix B.

One important feature is the dependence of the sequences $X_{n}$ and $Z_{n}$ on $\Delta_{s p k}$ for fixed values of $\tau_{s t p}$. This highlights the fact the depression/facilitation-induced history-dependent filters are also frequencydependent.

A second important feature is that multiple combinations of $\tau_{d e p / f a c}$ and $\Delta_{s p k}$ give rise to the same sequence $X_{n}$ and $Z_{n}$, which from eqs. (7)-(8) depend on the ratios

$$
\gamma_{d}=\frac{\Delta_{s p k}}{\tau_{d e p}} \quad \text { and } \quad \gamma_{f}=\frac{\Delta_{s p k}}{\tau_{f a c}} .
$$

Constant values of $\gamma_{d}$ and $\gamma_{f}$ generate identical sequences $X_{n}$ and $Z_{n}$, respectively, which will be differently distributed in the time domain according to the rescaling provided by $\Delta_{s p k}$, reflecting the long-term time scales $\sigma_{d e p / f a c}$. This degeneracy also occurs for the steady state values $\bar{X}$ and $\bar{Z}$ (9)-(10), generating the $\bar{X}$ - and $\bar{Z}$-profiles (curves of $\bar{X}$ and $\bar{Z}$ as a function of the input frequency $1000 \Delta_{s p k}^{-1}$ ). This type of degeneracies may interfere with the inference process of short-term dynamics from experimental data.

A third important feature is that the update values $a_{d}$ and $a_{f}$ that operate at the single events contribute to the long-term time scale for the filters and do not simply produce a multiplicative effect on the filters uniformly across events.

\subsubsection{Descriptive envelope model for short-term dynamics in response to periodic presy- naptic inputs}

Following a similar approach as the one developed in Section 3.1 (for the synaptic dynamics with constant updates, independent of the presynaptic number) we extend the peak sequences (24)-(25) to the continuous domain

$$
X_{t}=\bar{X}+\left(1-a_{d}\right)^{t / \Delta_{s p k}} e^{-t / \tau_{d e p}}(1-\bar{X})
$$

and

$$
Z_{t}=\bar{Z}\left[1-\left(1-a_{f}\right)^{t / \Delta_{s p k}} e^{-t / \tau_{f a c}}\right] .
$$

The dynamics for $X_{t}$ and $Z_{t}$ are 1D, but more complex than the linear 1D dynamics for $S_{t}$ described in Section 3.1. In contrast to the dynamics of $S_{t}$ in Section 3.1, the filter and single-event dynamics for depression and facilitation are different. In particular, the time constant for the depression and facilitation filters involves the parameters $a_{d}$ and $a_{f}$, respectively, which are absent in the single-event dynamics. 
A1

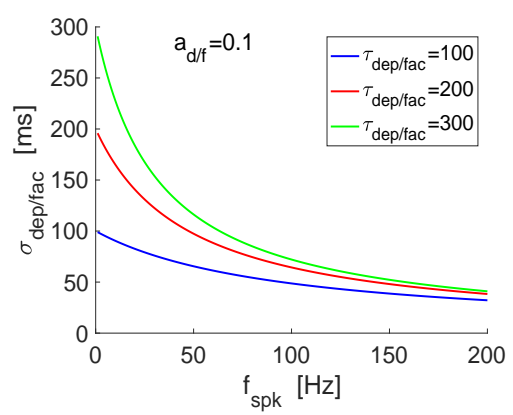

C1

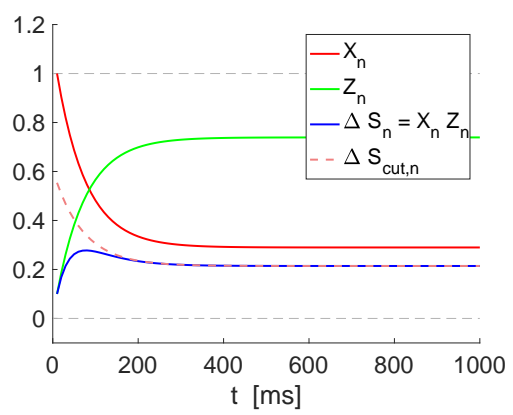

A2

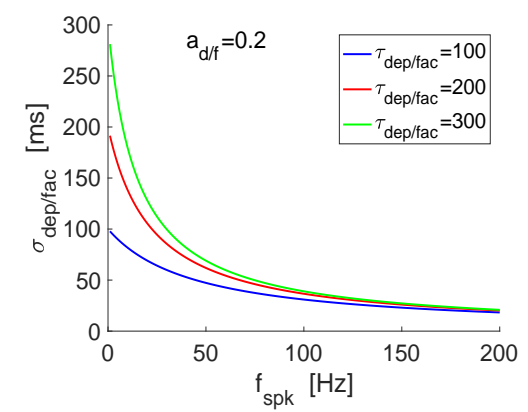

C2

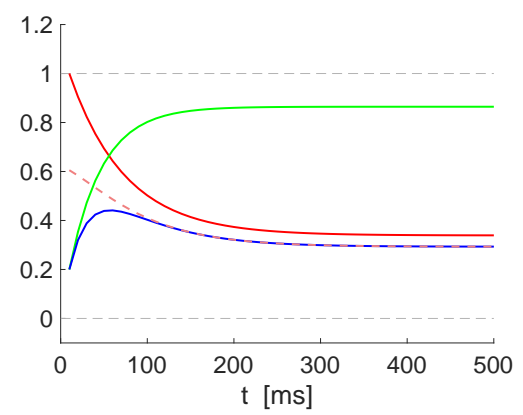

B

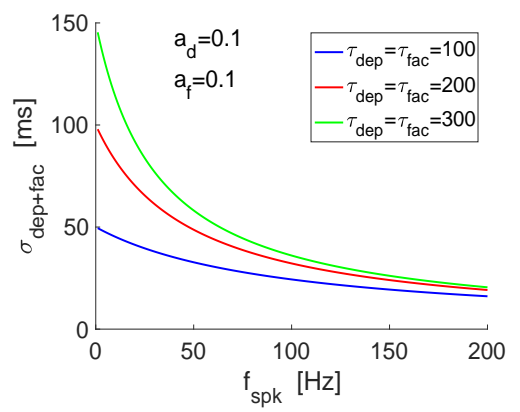

C3

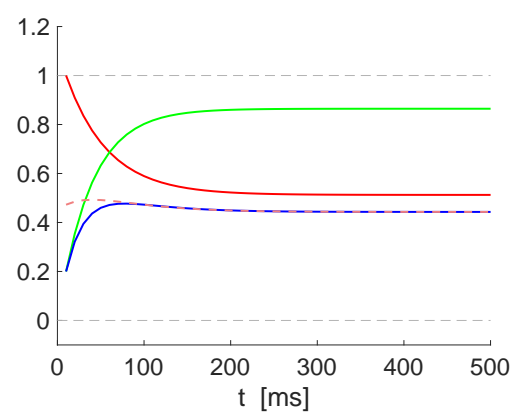

Figure 5: The time scales for the peak envelope responses $X_{n}$ and $Z_{n}$ to periodic presynaptic spikes and $\Delta S_{n}=X_{n} Z_{n}$ temporal filters $\left(\sigma_{d e p}, \sigma_{f a c}\right.$ and $\left.\sigma_{d e p+f a c}\right)$ depend on the interplay of $\tau_{d e p}, \tau_{f a c}$ and $f_{s p k}$ (or $\Delta_{s p k}$ ). The evolution of the peak sequences $X_{n}$ (depression, red) and $Z_{n}$ (facilitation, green), respectively are governed by eqs. (24)-(26) and the sequence $Q_{n}=Q\left(a_{s t p}, \tau_{s t p}\right)^{n-1}$ (light gray) is given by eq. (26). A. Dependence of the (long-term) temporal filter time constants $\sigma_{d e p}$ and $\sigma_{f a c}$ with the presynaptic input frequency $f_{s p k}$ and (short-term) time constants for the single events $\tau_{d e p}$ and $\tau_{f a c}$. We used eq. (28) with $\tau_{s t p}$ and $a_{s t p}$ substituted by $\tau_{d e p / f a c t}$ and $a_{d / f}$, respectively. A1. $a_{d}=a_{f}=0.1$. A2. $a_{d}=a_{f}=0.2$. B. Dependence of the the (long-term) temporal filter time constants $\sigma_{d e p+f a c}$ with the presynaptic input frequency $f_{s p k}$ and (short-term) time constants for the single events $\tau_{d e p}$ and $\tau_{f a c}$. We used eq. (28). C. Comparison between the filters produced by the "cut" sequence $\Delta S_{c u t, n}$ (light coral) and the sequence $\Delta S_{n}$ (blue) for representative parameter values. A1. $a_{d}=0.1, a_{f}=0.1, \tau_{d e p}=250$ and $\tau_{f a c}=250$. A2. $a_{d}=0.1, a_{f}=0.2, \tau_{d e p}=200$ and $\tau_{f a c}=250$. A3. $a_{d}=0.1, a_{f}=0.2, \tau_{d e p}=100$ and $\tau_{f a c}=250$. We used the following additional parameter values: $x_{\infty}=1$ and $z_{\infty}=0$. 
Here we propose a further simplification consisting of a descriptive model that captures the response of the depression and facilitation variables to periodic presynaptic inputs. It is based on the observation of the shapes of $X_{n}$ and $Z_{n}$ in Fig. 1, which suggests an exponential decay of these sequences to $\bar{X}$ and $\bar{Z}$ similar to the evolution of $S$ in response to con periodic presynaptic inputs and a constant update given by (22), even though the form of $X_{t}$ and $Z_{t}$ indicate this evolution is not strictly exponential. From eqs. (24)-(26) for the DA model we know the evolution is not strictly exponential, but it involves additional factors in $Q\left(a_{d}, \tau_{d e p}\right)^{n-1}$ and $Q\left(a_{f}, \tau_{f a c}\right)^{n-1}$. Therefore, the model we describe here is simpler than the DA model described above. Although is simply descriptive, it allows us to understand certain ideas of how temporal (history-dependent) filters interact.

Assuming exponential decay, the peak dynamics can be captured by the following envelope functions

$$
F\left(t_{k}\right)=A+(1-A) e^{-\left(t_{k}-t_{1}\right) / \sigma_{d}}
$$

and

$$
G\left(t_{k}\right)=B\left[1-C e^{-\left(t_{k}-t_{1}\right) / \sigma_{f}}\right]
$$

for $X$ and $Z$, respectively where we are using the same notation for the time constants as for the DA model. The parameters can be computed from these sequences by matching the initial values $\left(F\left(t_{1}\right)=\right.$ $X_{1}$ and $\left.G\left(t_{1}\right)=Z_{1}\right)$, the steady steady state values $\left(\bar{X}=\lim _{k \rightarrow \infty} X_{k}\right.$ and $\left.\bar{Z}=\lim _{k \rightarrow \infty} Z_{k}\right)$ and intermediate values $\left(t_{c}, X_{c}\right)$ and $\left(t_{c}, Z_{c}\right)$ chosen to be in the range of fastest increase/decrease of the corresponding sequences ( $\sim 50 \%$ of the gap between the initial and steady state values). This gives

$$
\begin{gathered}
A=\bar{X}, \quad B=\bar{Z}, \quad C=\frac{\bar{Z}-a_{f}}{\bar{Z}}, \\
\sigma_{d}=\left(t_{c}-t_{1}\right) \ln ^{-1}\left(\frac{1-\bar{X}}{X_{c}-\bar{X}}\right) \quad \text { and } \quad \sigma_{f}=\left(t_{c}-t_{1}\right) \ln ^{-1}\left(\frac{\bar{Z} C}{\bar{Z}-Z_{c}}\right) .
\end{gathered}
$$

Fig. 1-B (solid) shows the plots of $F$ (red), $G$ (green) and $H=F G$ (blue) superimposed with the $X_{n}$, $Z_{n}$ - and $\Delta S_{n}$-sequence values. The error between the sequences and the envelope curves (using a normalized sum of square differences) is extremely small in both cases, consistent with previous findings [67].

\subsubsection{Communication of the single event time scales to the (long-term) history-dependent filters: envelope descriptive model}

Eqs. (35) provide the time scales $\sigma_{d}$ and $\sigma_{f}$ for the functions $F\left(t_{k}\right)$ and $G\left(t_{k}\right)$ (32)-(33) in terms of $\tau_{d e p / f a c}, \Delta_{s p k}, a_{d / f}$ and the other model parameters for the sequences $X_{n}$ and $Z_{n}$ through their steadystates $\bar{X}$ and $\bar{Z}$ values (9)-(10). In order to estimate these time scales for $X_{n}$ and $Z_{n}$ we compute the values of $t_{c}$ and $X_{c}$ and $Z_{c}$.

For $f_{s p k} \rightarrow 1$, both $X_{n}$ and $Z_{n}$ are almost constant, since the $x(t)$ and $z(t)$ have enough time to recover to their steady state values before the next input spike arrives, and therefore $\sigma_{d} \gg 1$ and $\sigma_{f} \gg 1$. In contrast, for $f_{s p k} \gg 1, x(t)$ and $z(t)$ have little time to recover before the next input spike arrives and therefore they rapidly decay to their steady state values. In the limit of $f_{s p k} \rightarrow \infty, \sigma_{d}=\sigma_{f}=0$. In between these two limiting cases, $\sigma_{d}$ and $\sigma_{f}$ are decreasing functions of $f_{s p k}$ (Figs. 4-A1 and -A2). For fixed-values of $f_{s p k}$, both $\sigma_{d}$ and $\sigma_{f}$ are increasing functions of $\tau_{d e p}$ and $\tau_{f a c}$, respectively.

\subsection{Dynamics of the interplay of the depression and facilitation variables $x$ and $z$ : Emergence of temporal band-pass filters for $\Delta S_{n}=X_{n} Z_{n}$}

Under certain conditions, the interplay of depression and facilitation generates temporal band-pass filters in response to periodic inputs for $\Delta S=x z$ (Fig. 1-A), which are captured by the sequence $\Delta S_{n}=X_{n} Z_{n}$ 
(Fig. 1-A2 and -C2). A band pass filter requires $X_{1} Z_{1}<\bar{X} \bar{Z}$ and the existence of a spike index $r$ such that $X_{r} Z_{r}>\bar{X} \bar{Z}$. This in turn requires that the two time constants $\tau_{d e p}$ and $\tau_{f a c}$ are such that they create the appropriate balance between the two temporal filter time constants $\sigma_{d e p}$ and $\sigma_{f a c}$ (or $\sigma_{d}$ and $\sigma_{f}$ ) to support a band-pass filter for the product of the low- and high-pass temporal filters.

\subsubsection{Descriptive rules for the generation of temporal (envelope) band-pass filters from the interplay of the temporal (envelope) low- and high-pass filters}

From a geometric perspective, temporal band-pass filters in response to periodic presynaptic inputs arise as the result of the product of two exponentially increasing and decreasing functions both decaying towards their steady-state (e.g., Fig. 6). At the descriptive level, this is captured by the temporal envelope functions $(F, G$ and $H=F G)$ discussed above whose parameters are not the result of a sequence of single events but are related to the biophysical model parameters by comparison with the developed temporal filters. These functions provide a geometric/dynamic way to interpret the generation of temporal filters in terms of the properties of depression (decreasing functions) and facilitation (increasing functions) in response to periodic inputs, although this interpretation uses the developed temporal filters and therefore is devoid from any biophysical mechanistic interpretation.

A

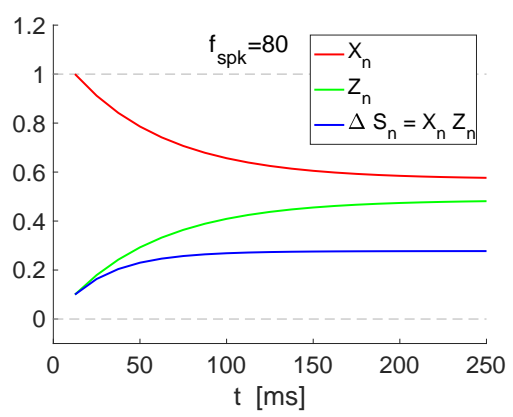

D

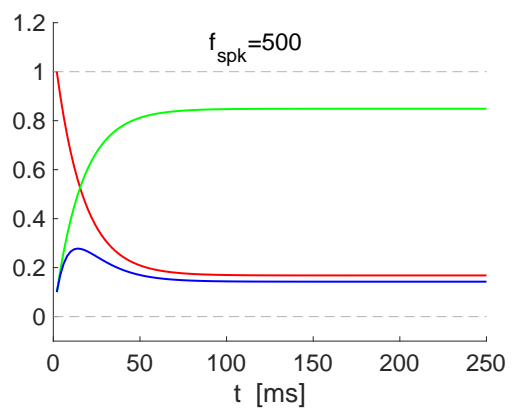

B

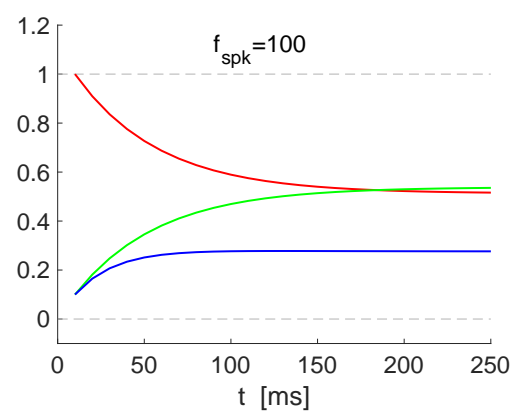

E

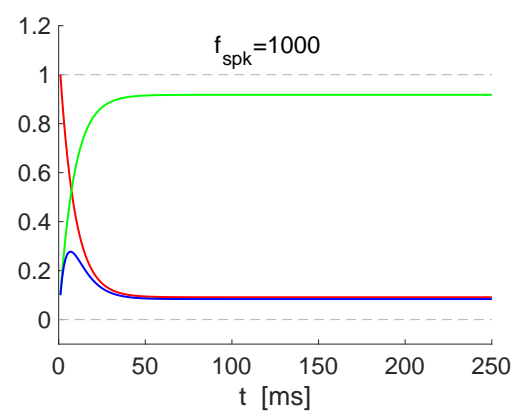

C

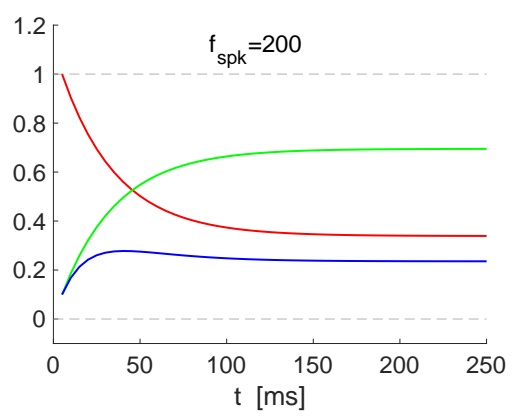

$\mathbf{F}$

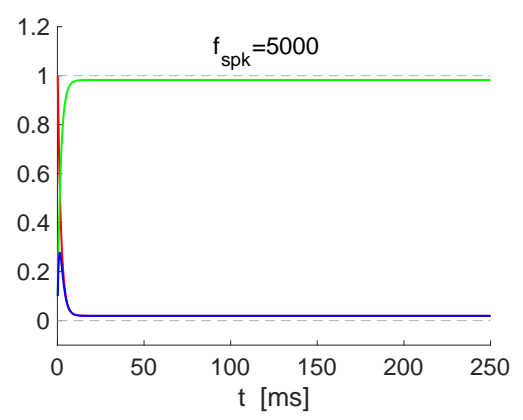

Figure 6: Transition from temporal high- to low-pass filters as the presynaptic frequency increases via a temporal bandpass filtering mechanism for fixed values of the synaptic depression and facilitation time constants. The evolution of the peak sequences $X_{n}$ (depression, red) and $Z_{n}$ (facilitation, green), respectively are governed by eqs. (24)-(26). We used the same parameter values for depression and facilitation: $\tau_{d e p}=\tau_{f a c}=100$ and $a_{d}=a_{f}=0.1$. A. $f_{s p k}=80$. B. $f_{s p k}=100$. C. $f_{s p k}=200$. D. $f_{s p k}=500$. E. $f_{s p k}=1000$. F. $f_{s p k}=5000$. We used the following additional parameter values: $x_{\infty}=1$ and $z_{\infty}=0$.

In order to investigate how the multiplicative interaction between $F(t)$ and $G(t)$ given by eqs. (32)-(33) give rise to the temporal band-pass filters $H=F G$, we consider a rescaled version of these functions 


$$
F(t)=A+(1-A) e^{-t / \eta}
$$

and

$$
G(t)=B\left[1-C e^{-t}\right]
$$

where $B=1$ and

$$
\eta=\frac{\sigma_{d}}{\sigma_{f}}
$$

The function $G$ transitions from $G(0)=1-C$ to $\lim _{t \rightarrow \infty} G(t)=1$ with a fixed time constant (Fig. 7, green curves). The function $F$ transitions from $F(0)=1$ to $\lim _{t \rightarrow \infty} F(t)=A$ with a time constant $\eta$ (Fig. 7, red curves). It follows that $H$ transitions from $H(0)=1-C$ to $\lim _{t \rightarrow \infty} H(t)=A B=A$ (Fig. 7, blue curves). A temporal band-pass filter is generated if $H$ raises above $A$ for a range of values of $t$. This requires $F$ to decay slow enough so within that range $H=F G>A$ (Fig. 7-A) or $A$ to be small enough (Fig. 7-B). In fact, as $A$ decreases, the values of $\eta$ required to produce a band-pass temporal filter increases (compare Fig. 7-A2 and -B2).

Changes in the parameter $B$ in (37) affect the height of the band-pass temporal filter, but not the generation mechanism described above. However, for certain ranges of parameter values $H$ is a temporal low-pass filter (not shown).

A1

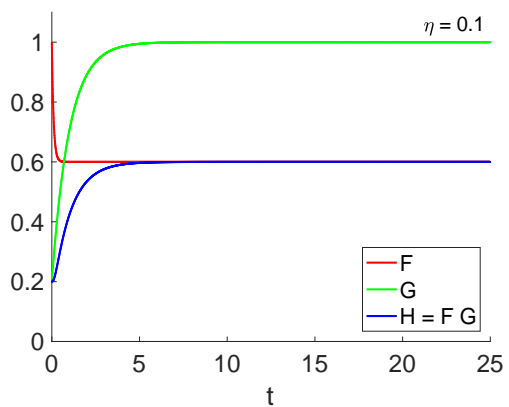

B1

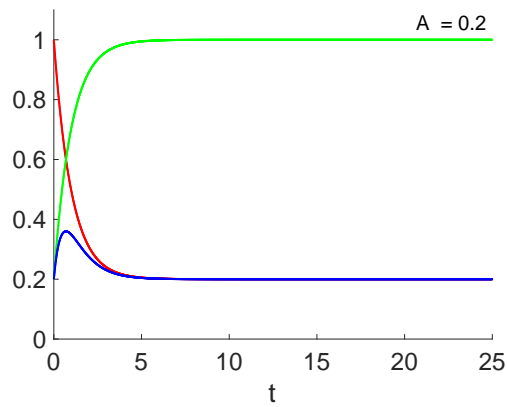

A2

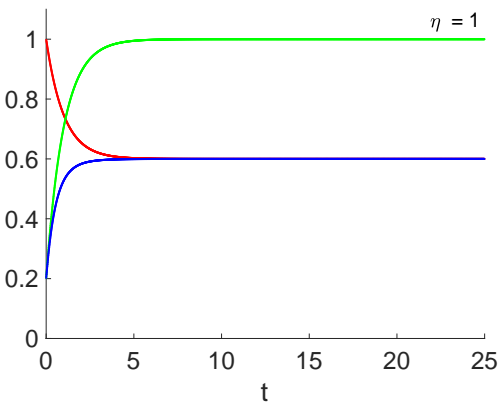

B2

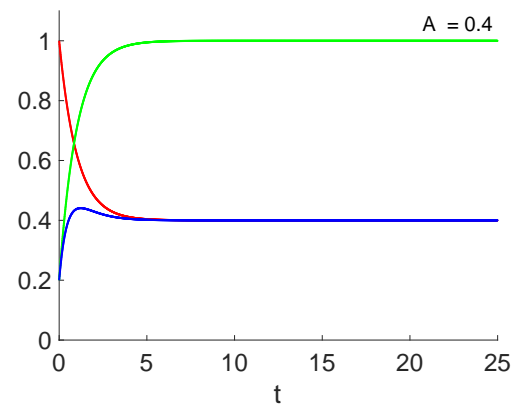

A3

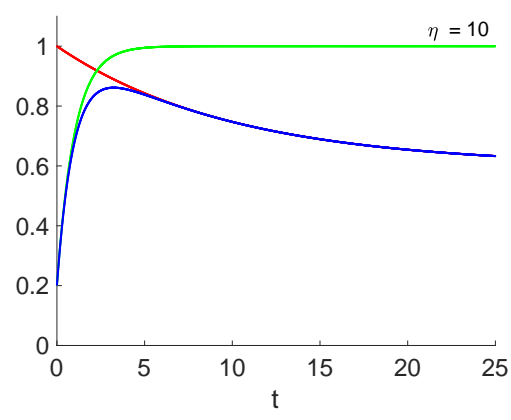

B3

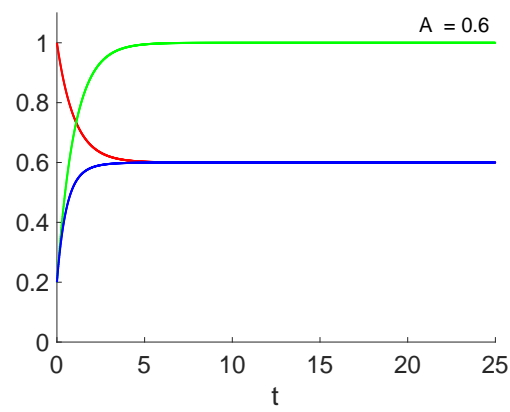

Figure 7: Temporal band-pass filters generated as the result of the multiplicative interaction of temporal low- and highpass filters: envelope functions approach. We used the envelope functions $F$ and $G$ defined by (44) and (37), respectively, and $H=F G$. A. Increasing $\eta$ contributes to the generation of a band-pass temporal filter. We used $A=0.5, C=0.8$ and $\mathbf{A} 1$. $\eta=0.1$. A2. $\eta=1$. A3. $\eta=10$. B. Decreasing $A$ contributes to the generation of a band-pass temporal filter. We used $\eta=1$, $C=0.8$ and B1. $A=0.2$. B2. $A=0.4$. B3. $A=0.6$. 


\subsubsection{Mechanisms of generation of temporal band-pass filters}

From a biophysically grounded mechanistic perspective, illustrated by Figs. 6 (see also Fig. 3-A), bandpass filters (panels $C, D, E$ ) provide a transition mechanism from low-pass filters for low enough input frequencies (panels $A$ and $B$ ) to high-pass filters for high-enough frequencies (panel $F$, which is strictly not a high-pass filters, but it is effectively so for the time scale considered). The depression and facilitation time constants $\tau_{d e p / f a c}$ modulate these transitions through the dependence of $X_{n}$ and $Z_{n}$ on the quotients $\Delta_{s p k} / \tau_{d e p / f a c}$ (Fig. 8-B to -D).

A1

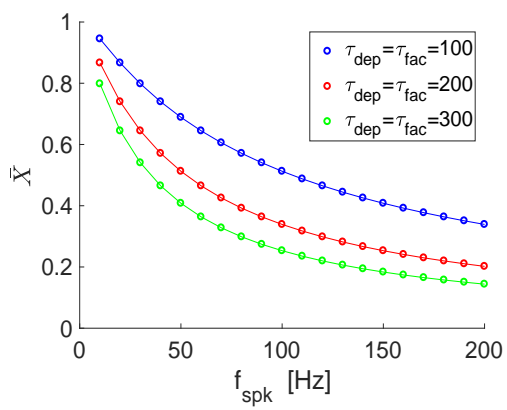

B1

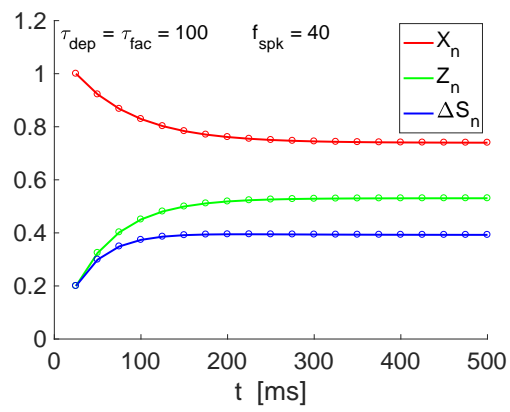

B2

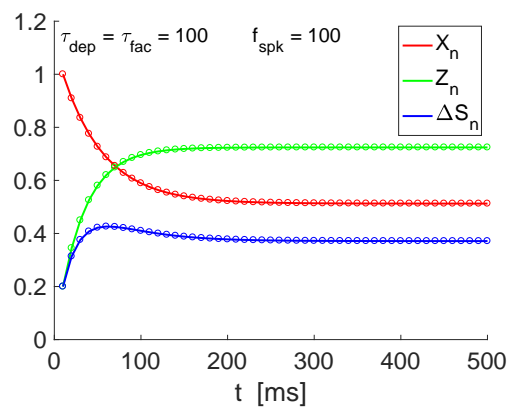

A2

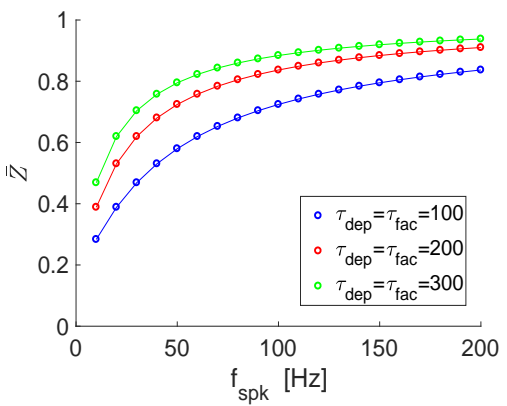

C1

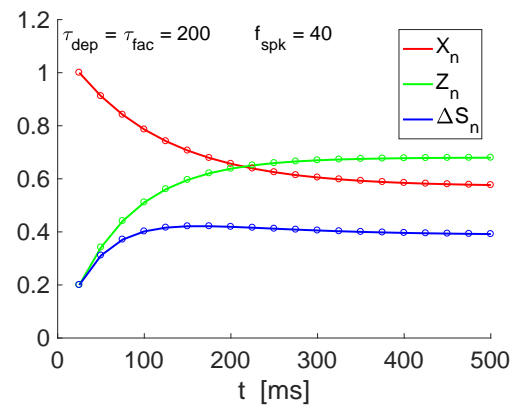

C2

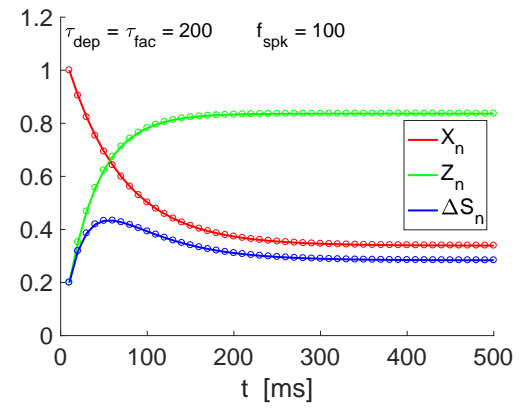

A3

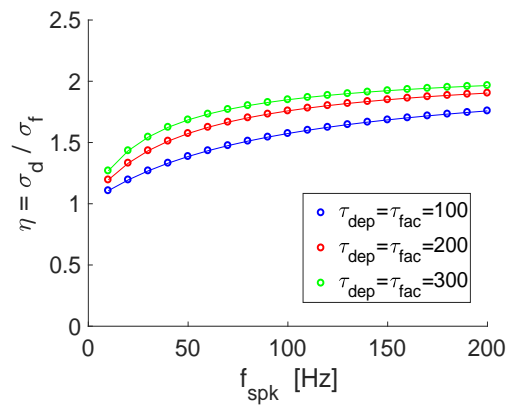

D1

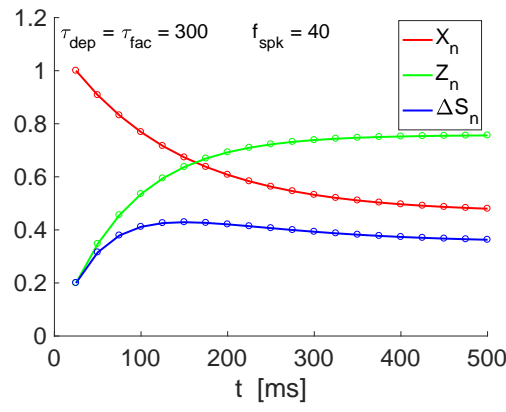

D2

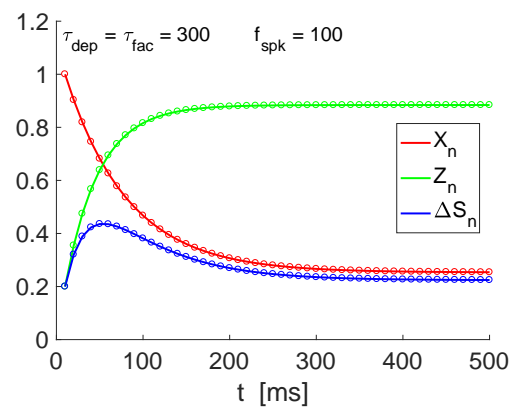

Figure 8: Temporal band-pass filters generated as the result of the multiplicative interaction of temporal low- and highpass filters: Peak envelope responses to periodic presynaptic inputs. A. Dependence of $\eta=\sigma_{d e p} / \sigma_{f a c}, \bar{X}$ and $\bar{Z}$ with the presynaptic input frequency $f_{s p k}$. We used formulas (9)-(10) together with (35) and the following parameter values: $a_{d}=0.1$, $a_{f}=0.2, x_{\infty}=1, z_{\infty}=0$.

By design, the geometric/dynamic mechanism described in the previous section is based on the assumption that the parameters $A, B, C, \sigma_{d}$ and $\sigma_{f}$ (or $\eta=\sigma_{d} / \sigma_{f}$ ) are free (and independent). There 
is no notion of single event dynamics within this framework. However, from (9)-(10) and (34)-(35) these parameters are not independent in the realistic model (e.g., they all depend on the presynaptic input frequency $f_{s p k}$ or period $\Delta_{s p k}$ ). Simple calculations show that $\bar{X}$ is a decreasing function of $f_{s p k}$ (e.g., Fig. 8-A1) (increasing function of $\Delta_{s p k}$ ) and $\bar{Z}$ is an increasing function of $f_{s p k}$ (e.g., Fig. 8-A2) (decreasing function of $\Delta_{s p k}$ ). The dependence of $\eta$ with $f_{s p k}$ is more complex and can be either increasing (e.g., Fig. 8-A3) or decreasing (not shown).

The behavior of $\bar{X}(=A), \bar{Z}(=B)$ and $\eta$ in Fig. 8 illustrates that $\Delta S_{n}$ temporal band-pass filters can be generated for these model parameters as $f_{s p k}$ increases (Figs. 8-B to -D), and it will be more pronounced as $\tau_{\text {dep }}=\tau_{f a c}$ increases (compare panels B1 to D1 and B2 to D2). Note that a $\Delta S_{n}$ temporal band-pass filter exists for $\tau_{d e p}=\tau_{f a c}=300$, but not for $\tau_{d e p}=\tau_{f a c}=100$, although $\tau_{d e p} / \tau_{f a c}=1$. Because of the dependence of $X_{n}$ and $Z_{n}$ on the quotients $\Delta_{s p k} / \tau_{d e p / f a c}, \Delta S_{n}$ temporal band-pass filters can be generated by increasing values of $\tau_{d e p}, \tau_{f a c}$ or both (not shown).

\section{5 $\Delta S_{n}$-band pass filters require a third (emergent) time scale whose contribu- tion is independent from the low- and high-pass filters' time scales}

The linear one-dimensional dynamics for both depression and facilitation at the (short-term) single event level generate linear one-dimensional discrete dynamics at the (long-term) temporal (low- and high-pass) filters level where the long-term time constants $\left(\sigma_{d}\right.$ and $\left.\sigma_{f}\right)$ depend on the short-term time constants $\left(\tau_{d e p}\right.$ and $\left.\tau_{f a c}\right)$ and the input frequency $\left(\Delta_{s p k}\right)$. From the discrete dynamics point of view, the temporal band-pass filters (BPFs) obtained as the product of the temporal LPF and HPF are overshoots where the sequence evolves by peaking at a higher value than the steady-state (without exhibiting damped oscillations). Overshoots require at least two-dimensional dynamics (generated by a difference equation where each value of the resulting sequence depends on the previous two) with the appropriate time scales. In [67] we showed that in certain circumstances the generation of temporal BPFs requires threedimensional dynamics. Here we investigate how the time scales giving rise to temporal BPF depend on the time scales of the temporal LPF and HPF and these of the corresponding single events.

\subsubsection{Mechanistic DA model}

From (24)-(26),

$$
\begin{aligned}
\Delta S_{n}=X_{n} Z_{n}= & \bar{X} \bar{Z}+Q\left(a_{d}, \tau_{d e p}\right)^{n-1}\left(X_{1}-\bar{X}\right) \bar{Z}+Q\left(a_{f}, \tau_{f a c}\right)^{n-1}\left(Z_{1}-\bar{Z}\right) \bar{X}+ \\
& +Q\left(a_{d}, \tau_{d e p}\right)^{n-1} Q\left(a_{f}, \tau_{f a c}\right)^{n-1}\left(X_{1}-\bar{X}\right)\left(Z_{1}-\bar{Z}\right) .
\end{aligned}
$$

The dynamics of the three last terms in eq. (53) are governed by $Q\left(a_{d}, \tau_{d e p}\right)^{n-1}, Q\left(a_{f}, \tau_{f a c}\right)^{n-1}$ and $\left[Q\left(a_{d}, \tau_{d e p}\right) Q\left(a_{f}, \tau_{f a c}\right)\right]^{n-1}$, respectively, and $\Delta S_{n} \rightarrow \bar{X} \bar{Z}$ as $n \rightarrow \infty$. The first two are given eq. (26) with $\tau_{s t p}$ substituted by $\tau_{d e p}$ and $\tau_{f a c}$, and $a_{s t p}$ substituted by $a_{d}$ and $a_{f}$, accordingly. The corresponding time scales are given by eq. (28) with the same substitutions. The last one is given by

$$
Q\left(a_{d}, \tau_{d e p}\right) Q\left(a_{f}, \tau_{f a c}\right)=\left(1-a_{d}\right)\left(1-a_{f}\right) e^{-\Delta_{s p k}\left(1 / \tau_{d e p}+1 / \tau_{f a c}\right)} .
$$

The product $\left[Q\left(a_{d}, \tau_{d e p}\right) Q\left(a_{f}, \tau_{f a c}\right)\right]^{n-1}$ cannot be expressed in terms of a linear combination of $Q\left(a_{d}, \tau_{d e p}\right)^{n-1}$ and $Q\left(a_{f}, \tau_{f a c}\right)^{n-1}$ and therefore the four terms in (53) are linearly independent.

The time scale associated to the fourth terms in (53) can be computed from (54) (as we did before) by calculating the time it takes for $\left[Q\left(a_{d}, \tau_{d e p}\right) Q\left(a_{f}, \tau_{f a c}\right)\right]^{n-1}$ to decrease from $\left[Q\left(a_{d}, \tau_{d e p}\right) Q\left(a_{f}, \tau_{f a c}\right)\right]^{n-1}$ from 1 (is value for $n=1$ ) to 0.37 and multiply this number by $\Delta_{s p k}$. This yields

$$
\sigma_{d e p+f a c}=\frac{\ln (0.37) \Delta_{s p k}}{\ln \left(1-a_{d}\right)+\ln \left(1-a_{f}\right)-\Delta_{s p k}\left(1 / \tau_{d e p}+1 / \tau_{f a c}\right)} \sim
$$




$$
\sim \frac{1}{\left(1 / \tau_{d e p}+1 / \tau_{f a c}\right)-\ln \left[\left(1-a_{d}\right)\left(1-a_{f}\right)\right] / \Delta_{s p k}} . .
$$

This long-term time scale has similar properties as $\sigma_{d e p}$ and $\sigma_{f a c}$. In particular, for $f_{s p k} \rightarrow 0$, $\sigma_{d e p+f a c} \rightarrow \tau_{d e p} \tau_{f a c} /\left(\tau_{d e p}+\tau_{f a c}\right)$. For $f_{s p k} \rightarrow \infty, \sigma_{d e p+f a c} \rightarrow 0$. For values of $f_{s p k}$ in between, $\sigma_{d e p+f a c}$ decrease between these extreme values. This is illustrated in Fig. 5-B along the time other two times scales, $\sigma_{d e p}$ and $\sigma_{f a c}$ (Fig. 5-B).

To say that the three time scales $\left(\sigma_{d e p}, \sigma_{f a c}\right.$ and $\left.\sigma_{d e p+f a c}\right)$ are independent is equivalent to state that the dynamics is three dimensional, while the dynamics of the depression and facilitation sequences are one-dimensional. It also means that erasing one of the terms in $\Delta S_{n}$ is equivalent to projecting the three-dimensional signal into a two-dimensional space with the consequent loss of information if it does not provide a good approximation to the original signal, and this loss of information may in principle be the loss of the band-pass filter (overshoot). On the other hand, two-dimensional systems are able to produce overshoots. So the question arises of whether the signal $\Delta S_{n}$ without the last term (that combines the time scales of the two filters $X_{n}$ and $Z_{n}$ ) preserves the temporal band-pass filter and, if yes, under what conditions.

In order to test the necessity of this third time scale for the generation of temporal band-pass filters, we consider the "cut" sequence

$$
\Delta S_{c u t, n}=\bar{X} \bar{Z}+Q\left(a_{d}, \tau_{d e p}\right)^{n-1}\left(X_{1}-\bar{X}\right) \bar{Z}+Q\left(a_{f}, \tau_{f a c}\right)^{n-1}\left(Z_{1}-\bar{Z}\right) \bar{X}
$$

where $\Delta S_{c u t, 1}=\left(X_{1}-\bar{X}\right) \bar{Z}+Z_{1} \bar{X}$ and $\lim _{n \rightarrow \infty} \Delta S_{c u t, n}=\bar{X} \bar{Z}$. For $a_{d}=a_{f}$ and $\tau_{\text {dep }}=\tau_{f a c}$, $Q\left(a_{d}, \tau_{d e p}\right)=Q\left(a_{f}, \tau_{f a c}\right)$, and therefore $\Delta S_{c u t, n}$ has the same structure as $X_{n}$ and $Z_{n}$, and therefore $\Delta S_{c u t, n}$ cannot generate a temporal band-pass filter regardless of the value of $\Delta_{s p k}$. This includes the examples presented in Fig. 3. For other parameter values, standard calculations show that the parameter ranges for which $\Delta S_{c u t, n}$ shows a peak are very restricted and when it happens, they rarely provide a good approximation to the temporal band-pass filter exhibited by $\Delta S_{n}$. Fig. 5-C illustrates this for a number of representative examples.

\subsubsection{Descriptive envelope model}

Here we address similar issues in the descriptive models described in the previous section. We consider the function

$$
H\left(t_{k}\right)=F\left(t_{k}\right) G\left(t_{k}\right)
$$

which approximates the behavior of $\Delta S_{n}$. From (32)-(33),

$$
H\left(t_{k}\right)=\bar{Z}\left[\bar{X}+(1-\bar{X}) e^{-\left(t_{k}-t_{1}\right) / \sigma_{d}}-\bar{X} C e^{-\left(t_{k}-t_{1}\right) / \sigma_{f}}-(1-\bar{X}) C e^{-\left(t_{k}-t_{1}\right) / \sigma_{d+f}}\right]
$$

where

$$
\sigma_{d+f}=\left(\frac{1}{\sigma_{d}}+\frac{1}{\sigma_{f}}\right)^{-1}
$$

and $\sigma_{d}$ and $\sigma_{f}$ are given by (35). Note that $\sigma_{d}, \sigma_{f}$ and $\sigma_{d+f}$ are different quantities from $\sigma_{d e p}, \sigma_{f a c}$ and $\sigma_{d e p+f a c}$ discussed above.

This shows, from a different perspective than in the previous section, that the dynamics of $\Delta S_{n}$ involves three time scales: the two times scales discussed above $\left(\sigma_{d}\right.$ and $\left.\sigma_{f}\right)$ and the combined time scales $\sigma_{d+f}$ (Fig. 4-A3).

Together, these results and the results from the previous section shows that while $X_{n}$ and $Z_{n}$ are generated by a 1D (linear) discrete dynamical systems (1D difference equations), $\Delta S_{n}$ is generated by a 3D (linear) discrete dynamical system (3D discrete difference equation). Under certain conditions, a 2D 
(linear) discrete dynamical system will produce a good approximation. $\Delta S_{n}$ is able to exhibit a band-pass filter because of the higher dimensionality of its generative model.

In order to understand the contribution of the combined time scale $\sigma_{d+f}$, we look at the effect of cutting the fourth term in $\mathrm{H}$ (43). We call this function $H_{\text {cut }}$. Fig. 4-B shows that $H_{\text {cut }}$ does not approximate $\Delta S_{n}$ well during the transients (response to the first input spikes) and fails to capture the transient peaks and the temporal band-pass filter properties of $\Delta S_{n}$. This discrepancy between $\Delta S_{n}$ (or $\mathrm{H}$ ) and $H_{\text {cut }}$ is more pronounced for low than for high input frequencies. In fact $H_{c u t}\left(t_{1}\right)=\bar{Z}-\bar{X}\left(\bar{Z}-a_{f}\right)$, while $H\left(t_{1}\right)=a_{f}$. The question remains of whether there could be a 2D (linear) dynamical system able to reproduce the temporal filters for $\Delta S_{n}$ with (emergent) time scales different from $\sigma_{d}$ and $\sigma_{f}$. In other words, whether $\Delta S_{n}$ can be captured by a function of the form

$$
H_{2 D}\left(t_{k}\right)=h_{0}+h_{1} e^{-\left(t_{k}-t_{1}\right) / \sigma_{1}}+h_{2} e^{-\left(t_{k}-t_{1}\right) / \sigma_{2}} .
$$

where $h_{0}, h_{1}, h_{2}, \sigma_{1}$ and $\sigma_{2}$ are constants where by necessity,

$$
\lim _{t \rightarrow \infty} H_{2 D}\left(t_{k}\right)=h_{0}=\bar{X} \bar{Z} \quad \text { and } \quad H_{2 D}\left(t_{1}\right)=h_{0}+h_{1}+h_{2}=H\left(t_{1}\right)=a_{f} .
$$

\subsection{Interplay of short-term synaptic and cellular postsynaptic dynamics: tempo- ral BPFs generated within and across levels of organization}

Standard models of synaptic transmission consider synaptic currents such as eq. (2). The synaptic function $S$, which controls the synaptic efficacy, obeys a first linear kinetic equation such as eq. (92) ( $\kappa=\Delta S=1)$ in the Appendix C $[72,73]$ and saturates at $S=1$. The raise time $\tau_{r s e}$ is typically very fast, at least an order of magnitude faster than the decay time $\left(\tau_{d e c}\right)$ and therefore some simplified models consider an instantaneous increase right after the arrival of a presynaptic spike and a subsequent decay governed by an eq. such as eq. (3) with $\Delta S_{n}=1$ for all $n$. Consistent with the saturation principle, the update should be an increment to $S=1$. From this perspective, the dynamics discussed in Sections 2.1.7 and 3.1 would not be strictly applicable for synaptic functions since the update considered there is an increment by $\Delta S=1$ (and not to $\Delta S=1$ ). More specifically, temporal summation is not expected for the synaptic variable $S$. On the other hand, the model with an update by $\Delta S=1$ (Sections 2.1.7 and 3.1 ) is applicable as a simplified model of post-synaptic potential (PSP) response to presynaptic inputs if $\tau_{d e c}$ is interpreted as the membrane potential decay time.

Earlier models of synaptic dynamics consider the PSP peak sequence to be proportional to $\Delta S_{n}=$ $X_{n} Z_{n}[21,58,63]$. This approach does not take into consideration the dynamic interaction between the synaptic function $S$ and the membrane potential. While the synaptic dynamics with the update to $S=1$ reproduces the peak pattern of $\Delta S_{n}$, in these models, the PSP response does not reflect the effects of the membrane time scale. Later models consider the synaptic function $S$ embedded in the current-balance equation and obeying equations of the form (92) where the rate of $S$ increase (rise time) is proportional to $\Delta S_{n}$ [74] or a similar model with $\kappa=\Delta S=1$, but with the saturation value equal to a function of $\Delta S_{n}$ [64] (see Appendix C). The presence of additional times scales further in the line (e.g., membrane potential time scale) gives rise to the phenomenon of temporal summation and the associated HPF in response to periodic synaptic inputs in the absence of STD (see Fig. 2-B).

In this paper, we follow a dual approach to investigate the mechanisms of generation of PSP temporal filters in response to periodic inputs. We first consider postsynaptic dynamics governed by eq. (2) with $S$ increased by $\Delta S_{n}$ and extensions where the decay time reflects the membrane potential dynamics. This model has the advantage of being analytically solvable and it allows us to understand the effects of the filtering (depression, facilitation and summation) time scales in terms of the single event time constants $\left(\tau_{d e p}, \tau_{f a c}\right.$ and $\left.\tau_{d e c}\right)$. Then, we use the more biophysically realistic approach by using eqs. (1)-(3) with $S$ increased to $\Delta S_{n}$ at the arrival of each presynaptic spike. In this model the interplay between the synaptic input and the postsynaptic cell is multiplicative (nonlinear). 


\subsubsection{PSP temporal filters in the simplified model}

We use eq. (3) with an update by $\Delta S_{n}$. Here $S$ is interpreted as an intermediate between the synaptic and the postsynaptic response with longer time scales than the standard AMPA and GABA ${ }_{A}$ synapses, reflecting the membrane potential time scales of postsynaptic cells. Our goal is to understand how the (global) time scales of the $S$-response patterns to periodic inputs depend on the time constant $\tau_{d e c}$ and the depression and facilitation time scales $\tau_{d e p}$ and $\tau_{f a c}$ through the $\Delta S_{n}$ filter time scales $\sigma_{d e p}$ and $\sigma_{f a c}$.

In the absence of depression and facilitation $\left(\tau_{d e p}, \tau_{f a c} \rightarrow 0\right), \Delta S_{n}$ is constant across cycles and $S$ generates temporal summation HPFs as described in Section 3.1 (Fig. 2-B) with $\sigma_{\text {sum }}=\tau_{\text {dec }}$. We use the notation $S_{n}^{0}$ for the corresponding sequences. In the presence of either depression or facilitation, the update $\Delta S_{n}$ is no longer constant across cycles and therefore, the STD LPFs and HPFs interact with the summation HPFs. From a different perspective, the inputs to $S$ are no longer constant across cycles but modulated by STD.

By solving the differential equation (3) where $S$ is increased by $\Delta S_{n}$ at the arrival of each spike $\left(t=t_{n}\right.$, $\left.n=1, \ldots, N_{s p k}\right)$ one arrives to the following discrete linear differential equation for the peak sequences in terms of the model parameters

$$
S_{n+1}=e^{-\Delta_{s p k} / \tau_{d e c}} S_{n}+\Delta S_{n+1} .
$$

The solution to eq. (47) is given by the following equation involving the convolution between the STP input $\Delta S_{n}$ and an exponentially decreasing function

$$
S_{n}=e^{-(n-1) \Delta_{s p k} / \tau_{d e c}} S_{1}+\sum_{k=0}^{n-2} e^{-k \Delta_{s p k} / \tau_{d e c}} \Delta S_{n-k}
$$

with $S_{1}=\Delta S_{1}=a_{f}$. The evolution of $S_{n}$ is affected by the history of the STP inputs $\Delta S_{n}$ weighted by an exponentially decreasing function of the spike index and a coefficient

$$
\gamma_{d e c}=\frac{\Delta_{s p k}}{\tau_{d e c}} .
$$

The steady state is given by

$$
\bar{S}=\left(\sum_{k=0}^{\infty} e^{-k \Delta_{s p k} / \tau_{d e c}}\right) \Delta^{-} S=\frac{\overline{\Delta S}}{1-e^{-\Delta_{s p k} / \tau_{d e c}}},
$$

where $\overline{\Delta S}=\bar{X} \bar{Z}$ given by (9) and (10). Note that Eq. (19) is a particular case of eq. (50) when $\Delta S_{n}$ is a constant sequence (no STD).

Both $S_{n}$ and $\bar{S}$ depend on $\Delta_{s p k}$ and the time constants $\tau_{d e p}, \tau_{f a c}$ and $\tau_{d e c}$ through the quotients (29) and (49). Therefore, here we consider temporal patterns for a fixed-value of $\Delta_{s p k}$. The temporal patterns for other values of $\Delta_{s p k}$ will be temporal compressions, expansions and height modulations of these baseline patterns. The values of $\tau_{d e p}, \tau_{f a c}$ and $\tau_{d e c}$ used in our simulations should be interpreted in this context.

For the limiting case $\tau_{d e c} \rightarrow 0, S_{n} \rightarrow \Delta S_{n}$ for $1=2, \ldots, N_{s p k}$. The $S$-temporal filter reproduces (is equal to or a multiple of) the $\Delta S_{n}$ as in $[21,58,63]$. For the limiting case $\tau_{d e c} \rightarrow \infty, \bar{S} \rightarrow \infty$ reflecting the lack of convergence of the sum in eq. (48). As the presynaptic spike number increases, the $S$-temporal filter reproduces the $S^{0}$-temporal filter since the $\Delta S_{n} \rightarrow \Delta^{-} S$. In the remaining regimes, changes in $\tau_{d e c}$ affect both the steady state and the temporal properties of $S$ in an input frequency-dependent manner as the $S$-temporal filter transitions between the two limiting cases. The former will be the object of a separate study. Here we focus on the temporal filtering properties. For visualization purposes and to compare the (global) time constants of the $S, \Delta S$ and $S^{0}$ temporal filters, we present the $S_{n}, \Delta S_{n}$ and $S_{n}^{0}\left(\tau_{d e p}, \tau_{f a c} \rightarrow 0\right)$ curves normalized by their maxima. 


\section{Emergence of $S_{n}$ temporal BPFs: Interplay of synaptic depression (LPF) and postsynap- tic summation (HPF)}

In the $\Delta S_{n}$ facilitation-dominated regime (Fig. 9-A), the PSP $S_{n}$ patterns result from the interaction between two HPFs, the $\Delta S_{n}$ facilitation and the $S_{n}^{0}$ summation ones. The filter time constant increases with increasing values of $\tau_{d e c}$ reflecting the dependence of the summation (global) time constant $\sigma_{\text {sum }}$ with $\tau_{\text {dec }}$.

A1

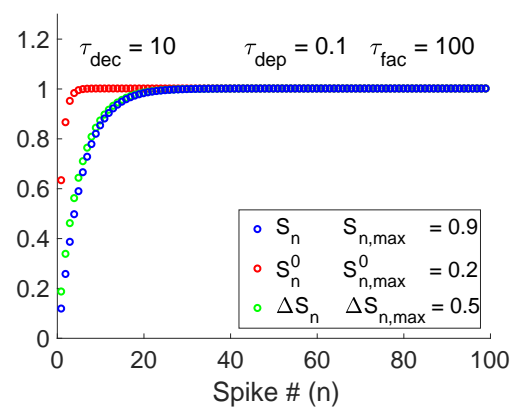

B1

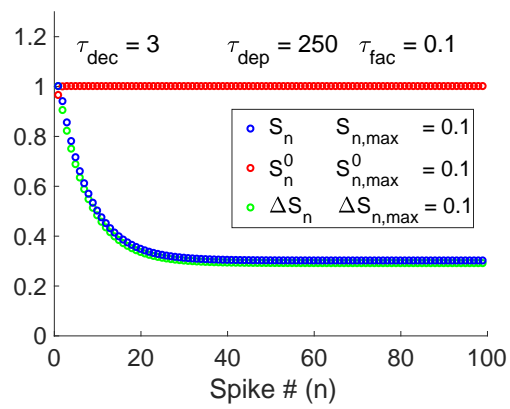

C1

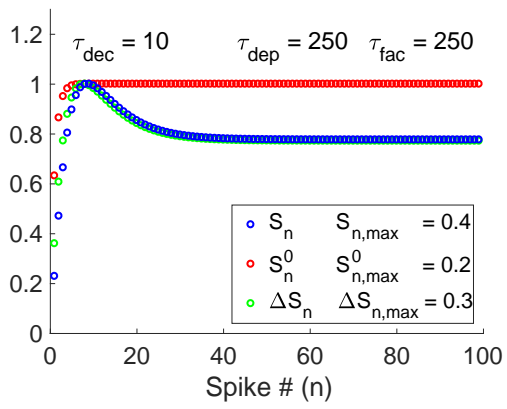

A2

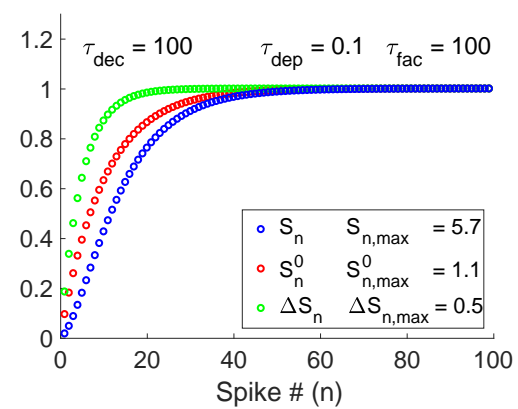

B2

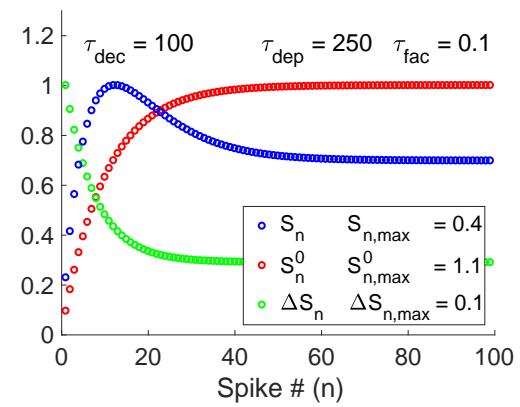

C2

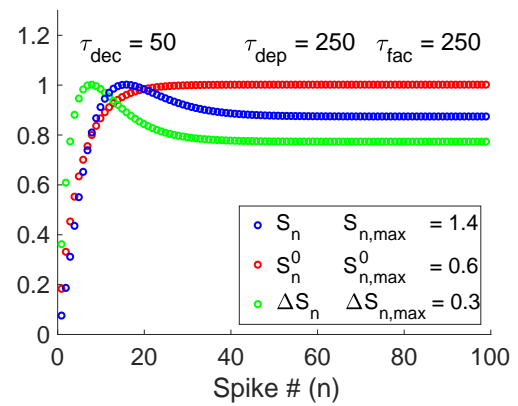

A3

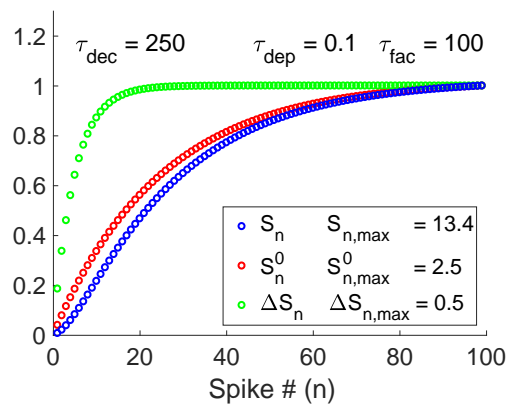

B3

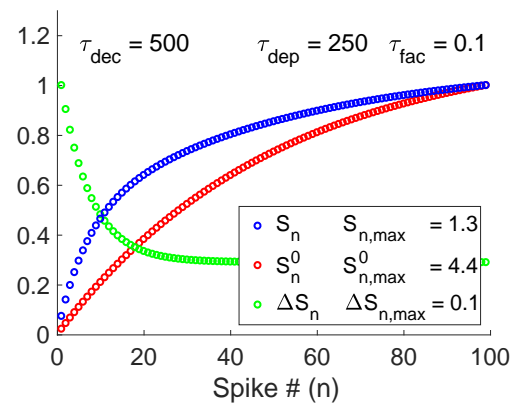

C3

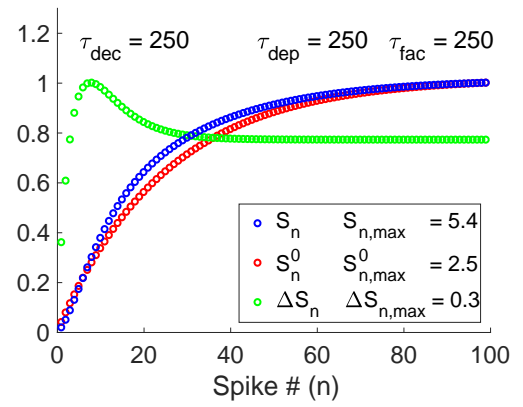

Figure 9: Temporal $S$-filters in response to periodic presynaptic inputs in the presence of STD. We used the DA model for synaptic depression and facilitation to generate the sequences $\Delta S_{n}$ and eq. (3) to generate the $S_{n}$ sequences. The $S_{n}^{0}$ sequences were computed using eq. (3) with a constant value of $\Delta S_{n}=\Delta S=a_{f a c}$. The curves are normalized by their maxima $S_{n, \max }$, $\Delta S_{n, \max }$ and $S_{n, \max }^{0}$. A. Facilitation-dominated regime. $S_{n}$ is a HPF (transitions between two HPFs). The time constant increases monotonically with $\tau_{d e c}$. B. Depression-dominated regime. A $S_{n}$ BPF is created as the result of the interaction between the presynaptic (depression) LPF and the temporal summation HPF. C. $S_{n}$ and $\Delta S_{n}$ temporal BPFs peak at different times. We used the following parameter values: $a_{d}=0.1, a_{f}=0.2, x_{\infty}=1, z_{\infty}=0$. 
In the $\Delta S_{n}$ depression-dominated regime (Fig. 9-B), temporal PSP $S_{n}$ BPFs emerge as the result of the interaction between the $\Delta S_{n}$ depression LPF and a $S_{n}^{0}$ HPF for intermediate values of $\tau_{\text {dec }}$ (Fig. 9-B2). $S_{n}$ BPFs are not present for small enough values of $\tau_{d e c}$ (Fig. 9-B1) since this would require $\Delta S_{n}$ to be a BPF, and are also not present for large enough values of $\tau_{d e c}$ (Fig. 9-B3) since this would require $S_{n}^{0}$ to be a BPF. As for the depression/facilitation BPFs discussed above, the $S_{n}$ BPFs are a balance between the two other filters and emerge as $S_{n}$ transitions in between them as $\tau_{d e c}$ changes.

\section{Dislocation of the (output) $S_{n}$ temporal BPF from the (input) $\Delta S_{n}$ temporal BPFs}

In this scenario, a depression-facilitation $\Delta S_{n}$ BPF is generated at the synaptic level and interacts with the summation $S_{n}^{0}$ HPF (Fig. 9-C). The $\Delta S_{n}$ BFP evokes a PSP $S_{n}$ BFP for low enough values of $\tau_{d e c}$ (Fig. 9-C1). As $\tau_{\text {dec }}$ increases, the $S_{n}$ pattern transitions to the $S_{n}^{0}$ HPF (Fig. 9-C3). As this transition happens, the $S_{n}$ BPF moves to the right and increases in size before entering the summation-dominated HPF regime. While the PSP $S_{n}$ BPF is inherited from the synaptic regime, its structure results from the interplay of the synaptic BPF and PSP temporal summation.

\subsubsection{Biophysically realistic models reproduce the above PSP temporal filters with sim- ilar mechanisms}

Here we test whether the results and mechanisms discussed above remain valid for the voltage response to periodic presynaptic inputs by using the more realistic, conductance-based model (1)-(3). Here $S$ has its original interpretation as a synaptic function with relatively small time constants, consistent with AMPA and $\mathrm{GABA}_{A}$ synapses. The value of $S$ is updated to $\Delta S_{n}=X_{n} Z_{n}$ upon the arrival of each spike (at a time $t_{n}$ ), capturing the effects of synaptic depression and facilitation produced by the DA model. Because the interaction between the synaptic variable $S$ and $V$ are multiplicative, the model is not analytically solvable.

The $V$ temporal patterns generated by this model (Fig. 10) are largely similar to the ones discussed above (Fig. 9) and are generated by similar mechanisms described in terms of the interplay of the membrane potential time scale $\tau_{m}(=C / g L)$ and the depression/facilitation time scales ( $\tau_{\text {dep }}$ and $\left.\tau_{f a c}\right)$ through the (global) $\Delta S_{n}$ filter time scales $\left(\sigma_{d e p}\right.$ and $\left.\sigma_{f a c}\right)$. Because $\tau_{d e c}$ is relatively small, $S$ largely reproduces the temporal properties of the $\Delta S_{n}$ pattern. As before, $V_{n}^{0}$ refer to the voltage response to presynaptic periodic inputs in the absence of STD ( $S$ is updated to $\Delta S_{n}$ constant). Fig. 10-A illustrates the generation of $V$ temporal BPFs as the result of the interaction between synaptic depression (LPF) and postsynaptic summation (HPF). Fig. 10-B illustrates the dislocation of postsynaptic BPF inherited from the synaptic input Fig. 10-A. We limited our study to realistic values of $\tau_{m}$.

\subsection{Interplay of multiple depression and facilitation process with different time scales}

In the models discussed so far both STD and STF involve one time scale ( $\tau_{d e p}$ and $\left.\tau_{f a c}\right)$ that governs the evolution of the corresponding variables $(x$ and $z)$ in between presynaptic spikes. These single-event time scales are the primary component of the temporal filter time scales $\left(\sigma_{d}\right.$ and $\left.\sigma_{f}\right)$ that develop in response to the periodic repetitive arrival of presynaptic spikes.

Here we extend these ideas to include scenarios where depression and facilitation involve more than one time scale. We interpret this as the coexistence of more than one depression or facilitation process whose dynamics are governed by a single single-event time scale each. Similar to the standard model, the independent filters that develop in response to the presynaptic spike train inputs interact to provide an input to the synaptic dynamics. In principle, this interaction may take various forms. Here, for exploratory purposes and to develop ideas, we consider a scenario where the processes of the same type 
A1

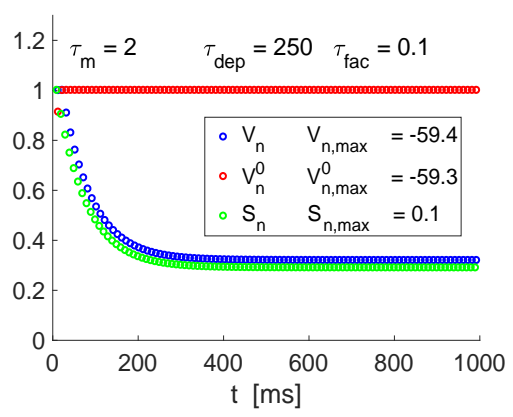

B1

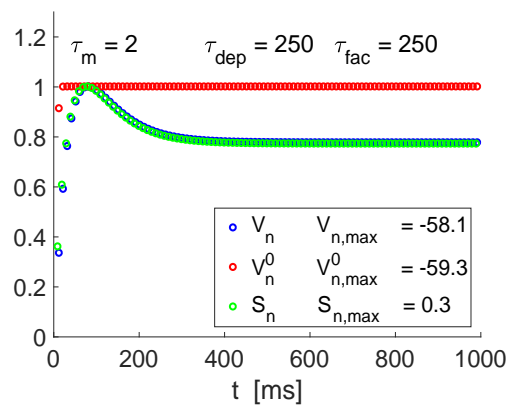

A2

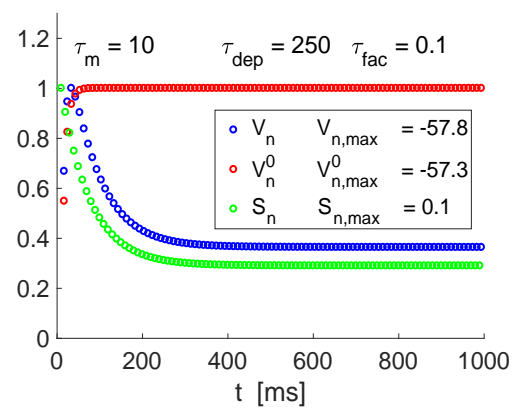

B2

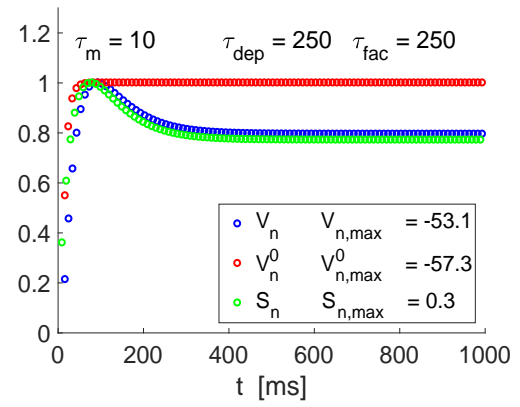

A3

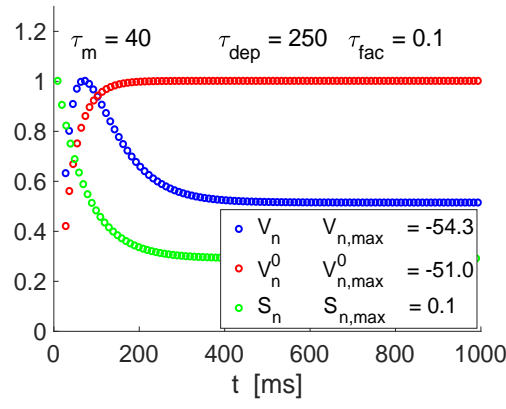

B3

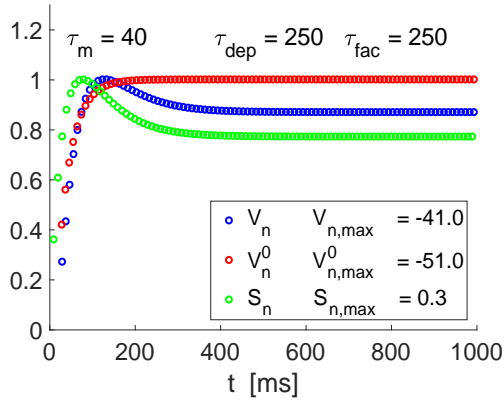

Figure 10: Temporal $V$-filters in response to periodic presynaptic inputs in the presence of STD. We used the DA model for synaptic depression and facilitation to generate the sequences $\Delta S_{n}$, eq. (3) $\left(\tau_{d e c}=5\right)$ to generate the $S_{n}$ sequences, and the passive membrane equation (1)-(2) to generate the $V_{n}$ sequences. The $V_{n}^{0}$ sequences were computed using eq. (3) with a constant value of $\Delta S_{n}=\Delta S=a_{f a c}$. The curves are normalized by their maxima $V_{n, \max }, \S_{n, \max }$ and $V_{n, \max }^{0}$. B. Depression-dominated regime. A $V_{n}$ BPF is created as the result of the interaction between the presynaptic (depression) LPF and the temporal summation HPF. C. $V_{n}$ and $S_{n}$ temporal BPFs peak at different times. We used the following parameter values: $a_{d}=0.1, a_{f}=0.2, x_{\infty}=1$, $z_{\infty}=0, C=1, E_{L}=-60, E_{\text {syn }}=0, G_{\text {syn }}=0.1$. 
(depression or facilitation) are linearly combined and the interaction between depression and facilitation is multiplicative as for the single depression-facilitation processes. We refer to it as the distributive or cross model. In the Appendix D we discuss other possible formulations. The ideas we develop here can be easily extended to more STD processes.

\subsubsection{The cross (distributive) model}

More specifically, the depression and facilitation variables $x_{k}$ and $z_{k}, k=1,2$ obey equations of the form (5)-(6) with parameters $\tau_{d e p, k}, \tau_{f a c, k}, a_{d, k}$ and $a_{f, k}$ for $k=1,2$. The evolution of these variables generate the sequences $X_{k, n}$ and $Z_{k, n}(k=1,2)$ given by 24)-(25) with the steady-state values $\bar{X}_{k}$ and $\bar{Z}_{k}(k=1,2)$ given by (9)-(10). For simplicity, we consider $a_{d, 1}=a_{d, 2}$ and $a_{f, 1}=a_{f, 2}$ (and omit the index $k$ ) so the differences between two depression or facilitation filters depend only on the differences of the single-event time constants. This can be easily extended to different values of these parameters for the different processes. In what follows, we will not specify the range of the index $k=1,2$ unless necessary for clarity.

In the cross (or distributive) model, the variable $M$ is given by

$$
M^{\times}(t)=\left[\eta_{d, 1} x_{1}(t)+\eta_{d, 2} x_{2}(t)\right]\left[\eta_{f, 1} z_{1}(t)+\eta_{f, 2} z_{2}(t)\right]
$$

where $\eta_{d, 1}+\eta_{d, 2}=1$ and $\eta_{f, 1}+\eta_{f, 2}=1$. Correspondingly, the synaptic update is given by

$$
\Delta S_{n}^{\times}=\left[\eta_{d, 1} X_{1, n}+\eta_{d, 2} X_{2, n}\right]\left[\eta_{f, 1} Z_{1, n}+\eta_{f, 2} Z_{2, n}\right]=\left(\sum_{k=1}^{2} \eta_{d, k} X_{k, n}\right)\left(\sum_{k=1}^{2} \eta_{f, k} Z_{k, n}\right)
$$

for $n=1, \ldots, N_{s p k}$. This model allows for all possible interactions between the participating depression and facilitation processes. It reduces to the single depression-facilitation process for $\eta_{d, 2}=\eta_{f, 2}=0$ (or $\eta_{d, 1}=\eta_{f, 1}=0$ ) and allows for independent reductions of depression and facilitation by making $\eta_{d, 2}=0$ or $\eta_{f, 2}=0$, but not both simultaneously.

From (24)-(26),

$$
X_{k, n}=\bar{X}_{k}+Q\left(a_{d}, \tau_{d e p, k}\right)^{n-1}\left(\hat{X}-\bar{X}_{k}\right)
$$

and

$$
Z_{k, n}=\bar{Z}_{k}+Q\left(a_{f}, \tau_{f a c, k}\right)^{n-1}\left(\hat{Z}-\bar{Z}_{k}\right)
$$

for $k=1,2$ with

$$
Q\left(a_{s t p}, \tau_{s t p, k}\right)=\left(1-a_{s t p}\right) e^{-\Delta_{s p k} / \tau_{s t p, k}},
$$

where for use the notation $\hat{X}$ and $\hat{Z}$ to refer to the first elements in the sequences, which, for simplicity, are assumed to be independent of $k$.

We use the notation

$$
\Delta S_{n}^{\times}=\Delta S_{d e p, n} \Delta S_{f a c, n},
$$

where after algebraic manipulation,

$$
\Delta S_{d e p, n}=\sum_{k=1}^{2} \eta_{d, k} \bar{X}_{k}+\left(1-a_{d}\right)^{n-1} \sum_{k=1}^{2} \eta_{d, k} e^{-(n-1) \Delta_{s p k} / \tau_{d e p, k}}\left(\hat{X}-\bar{X}_{k}\right)
$$

and 


$$
\Delta S_{f a c, n}=\sum_{k=1}^{2} \eta_{f, k} \bar{Z}_{k}+\left(1-a_{f}\right)^{n-1} \sum_{k=1}^{2} \eta_{f, k} e^{-(n-1) \Delta_{s p k} / \tau_{f a c, k}}\left(\hat{Z}-\bar{Z}_{k}\right)
$$

\subsubsection{Depression $\left(\Delta S_{d e p, n}\right)$, facilitation $\left(\Delta S_{f a c, n}\right)$ and $\Delta S_{n}^{\times}$temporal filters}

The history-dependent temporal filter $\Delta S_{d e p, n}$ transitions from $\Delta S_{d e p, 1}=\hat{X}$ to $\bar{S}_{d e p}=\eta_{d, 1} \bar{X}_{1}+\eta_{d, 2} \bar{X}_{2}$ as $n \rightarrow \infty$, and $\Delta S_{f a c, n}$ transitions from $\Delta S_{f a c, 1}=\hat{Z}$ to $\Delta^{-} S_{f a c}=\eta_{f, 1} \bar{Z}_{1}+\eta_{f, 2} \bar{Z}_{2}$ as $n \rightarrow \infty$. Because the individual filters are monotonic functions, the linearly combined filters represented by the sequences $\Delta S_{d e p, n}$ and $\Delta S_{f a c, n}$ are also monotonic functions lying in between the corresponding filters for the individual filter components (Figs. 11-A1 and 12-A1 for depression and Figs. 11-A2 and 12-A2 for facilitation). As a consequence, the $\Delta S_{n}^{\times}$filters also lie in between the product of the corresponding individual filter components $\Delta S_{1, n}$ and $\Delta S_{2, n}$ (Figs. 11-A3 and Figs. 12-A3). In these figures, all parameter values are the same except for $\tau_{d e p, 1}$ and $\tau_{f a c, 1}$, which are $\tau_{d e p, 1}=\tau_{f a c, 1}=100$ in Fig. 11 and $\tau_{d e p, 1}=\tau_{f a c, 1}=10$ in Fig. 12. In both figures, the values of the facilitation time constants are $\tau_{d e p, 2}=\tau_{f a c, 2}=1000$.

In Fig. 11-A3 both $\Delta S_{1, n}$ and $\Delta S_{2, n}$ are temporal BPFs peaking almost at the same time. Consequently $\Delta S_{n}^{\times}$is also temporal BPFs lying strictly in between the individual ones and peaking almost at the same time. In Fig. 12-A3, in contrast, $\Delta S_{1, n}$ is a temporal HPF, while $\Delta S_{2, n}$ is a temporal BPF. The resulting $\Delta S_{n}^{\times}$is also a temporal BFP, but the two temporal BPFs peak at different times.

\subsubsection{Communication of the single event time scales to the history-dependent filters}

In Section 3.3.2 we developed a descriptive envelope model for STD in response to periodic presynaptic inputs consisting of the functions $F(t)$ for depression, $G(t)$ for facilitation, and $H(t)=F(t) G(t)$ for the synaptic update, given by eqs. (32)-(34) and (43). By approximating the model parameters using the results of our simulations for $x(t)$ and $z(t)$, we computed the filter time constants $\sigma_{d}, \sigma_{f}$ using (35) and $\sigma_{d+f}=\left(\sigma_{d}^{-1}+\sigma_{f}^{-1}\right)^{-1}$. This approach is not strictly necessary for the DA model since the sequences $X_{n}$ and $Z_{n}$ can be computed analytically as well as the filter time constants $\sigma_{d e p}, \sigma_{f a c}$ and $\sigma_{d e p+f a c}$, which convey the same dynamic information as $\sigma_{d}, \sigma_{f}$ and $\sigma_{d+f}$. The calculation of these time constants is possible since the filter sequences involved a single $n$-dependent term. However, this is not the case for $\Delta S_{d e p, n}$ and $\Delta S_{f a c, n}$, which are linear combinations of $n$-dependent terms. On the other hand, the shapes of $\Delta S_{d e p, n}$ and $\Delta S_{f a c, n}$ suggest these filters can be captured by the descriptive model by computing the appropriate parameters using the results of our simulations. We use the notation $F_{d e p}$, $F_{f a c}$ and $H^{\times}(t)=F_{d e p}(t) F_{f a c}(t)$. The solid lines in Figs. 11-A1 to -A3 and 12-A1 to A3 confirm this. In particular, parameter values can be found so that $F_{d e p}(t), F_{f a c}(t)$ and $H^{\times}(t)$ provide a very good approximation to $\Delta S_{d e p, n}, \Delta S_{f a c, n}$ and $\Delta S_{n}^{\times}$, respectively (gray solid lines).

Using the descriptive model we computed the time constants $\sigma_{d}^{\times}, \sigma_{f}^{\times}$and $\sigma_{d+f}^{\times}=\left[\left(\sigma_{d}^{\times}\right)^{-1}+\left(\sigma_{f}^{\times}\right)^{-1}\right]^{-1}$. Figs. 11-B and 12-B (blue) show the dependence of these time constants with the single-event depression and facilitation time constant $\tau_{d e p, 2}\left(=\tau_{f a c, 2}\right)$ for two values of $\tau_{d e p, 1}\left(=\tau_{f a c, 1}\right)$ and $\Delta_{s p k}=10$ $\left(f_{s p k}=100\right)$. These results capture the generic model behavior via rescalings of the type (29). A salient feature is the non-monotonicity of the curves for $\sigma_{d}^{\times}, \sigma_{f}^{\times}$and $\sigma_{d+f}^{\times}$(blue) in contrast to the monotonicity of the curves for $\sigma_{d, 2}, \sigma_{f, 2}$ and $\sigma_{d+f, 2}$ for the depression and facilitation second component (red).

\subsubsection{Degeneracy}

The fact that the same type of descriptive envelope models such as the one we use here capture the dynamics of the temporal filters for both single and multiple depression and facilitation processes show it will be difficult to distinguish between them on the basis of data on temporal filters. In other words, the type of temporal filters generated by the DA model (single depression and facilitation processes) are 
A1

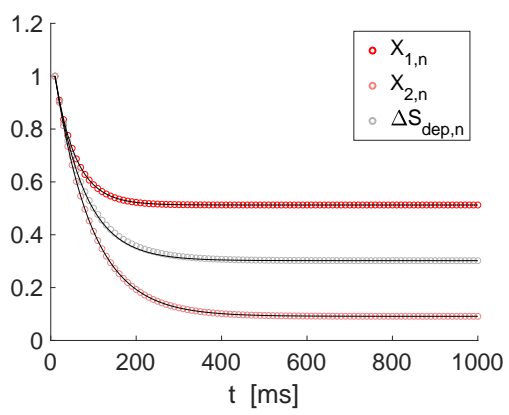

B1

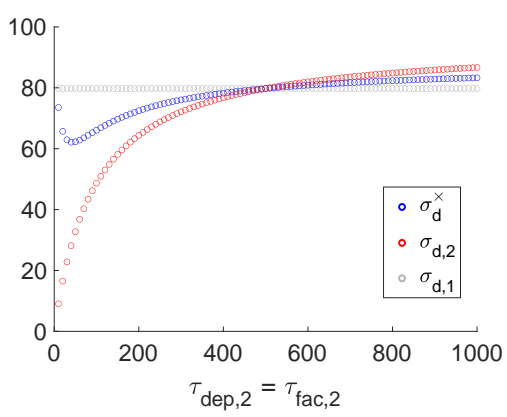

A2

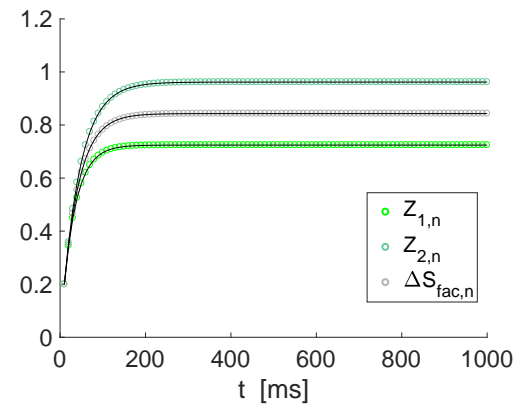

B2

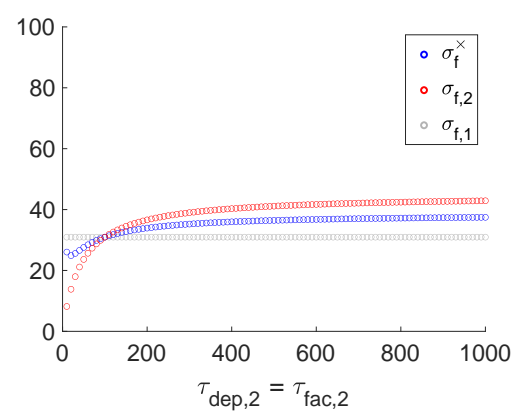

A3

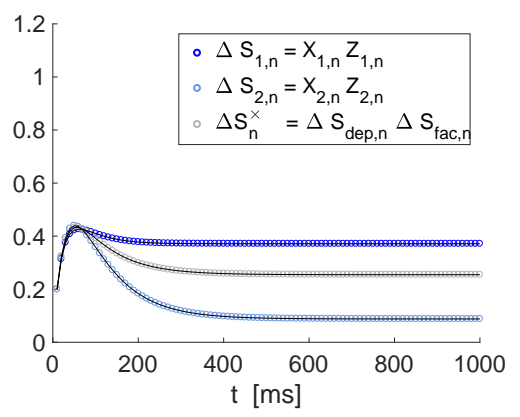

B3

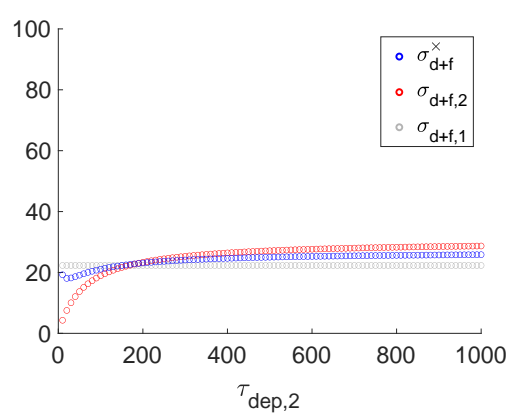

Figure 11: Temporal filters generated by the interplay of multiple depression and facilitation processes with different single-event time scales. A. Depression $(X)$, facilitation $(Z)$ and $\Delta S=X Z$ filters for representative parameter values. We use the distributive model (51)-(56) for the synaptic updates $\Delta S_{n}^{\times}$. The factors $\Delta S_{d e p, n}$ and $\Delta S_{f a c, n}$ in $\Delta S_{n}^{\times}$are given by eqs. (57)(58). The depression and facilitation individual filters $X_{k, n}$ and $Z_{k, n}(k=1,2)$ are given by eqs. (53)-(55). These and the $\Delta S_{d e p, n}$ and $\Delta S_{f a c, n}$ filters were approximated by using the descriptive envelope model for STD in response to periodic presynaptic inputs (solid curves superimposed to the dotted curves) described in Section 3.3.2 by eqs. (32)-(34). The filter $\Delta S_{n}^{\times}$was approximated by using with eq. (43) with $F$ and $G$ substituted by the corresponding approximations to $\Delta S_{d e p, n}$ and $\Delta S_{f a c, n}$. B. Dependence of the filter (global) time constants on the single events time constants. We used fixed values of $\tau_{d e p, 2}=\tau_{f a c, 2}$ and $\tau_{d e p, 1}$. Fig. 11 uses a different value of $\tau_{d e p, 1}$. The filter (global) time constants were computed using eq. (35). We used the following parameter values: $a_{d}=0.1, a_{f}=0.2, x_{\infty}=1, z_{\infty}=0, \tau_{d e p, 2}=\tau_{f a c, 2}=1000, \eta_{d e p}=\eta_{f a c}=0.5, \tau_{d e p, 1}=\tau_{f a c, 1}=100$, and $\Delta_{s p k}=10$. 
A1

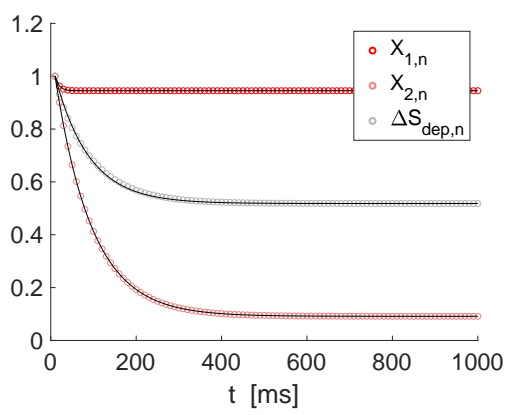

B1

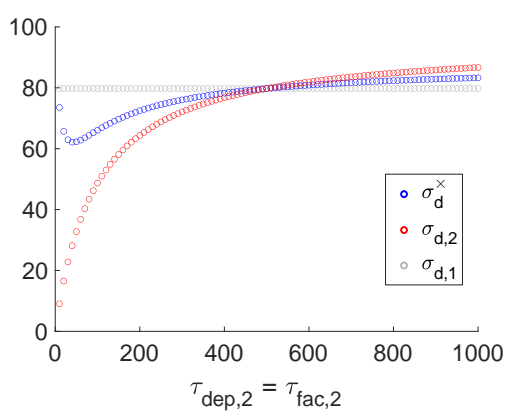

A2

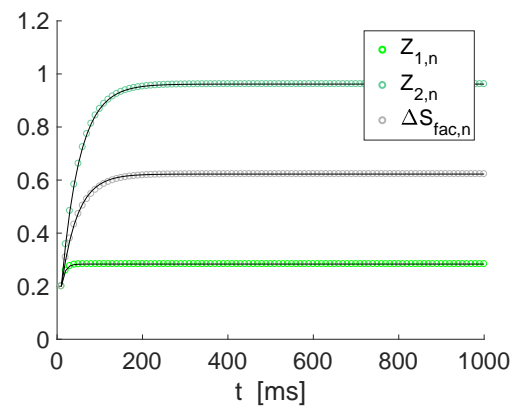

B2

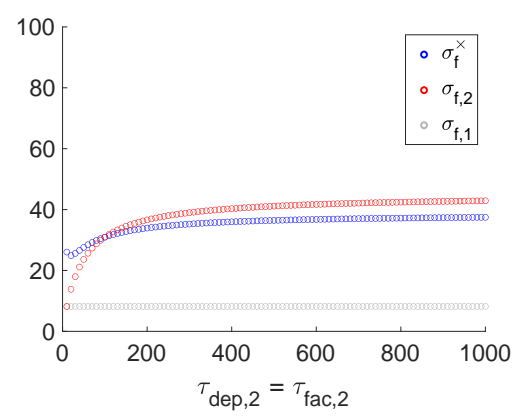

A3

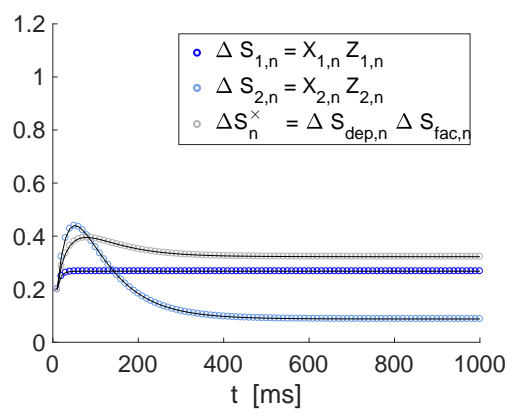

B3

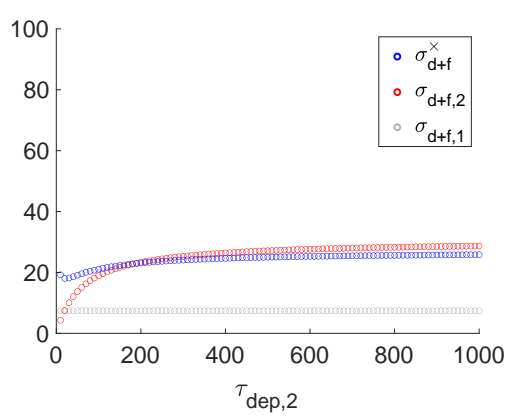

Figure 12: Temporal filters generated by the interplay of multiple depression and facilitation processes with different single-event time scales. A. Depression $(X)$, facilitation $(Z)$ and $\Delta S=X Z$ filters for representative parameter values. We use the distributive model (51)-(56) for the synaptic updates $\Delta S_{n}^{\times}$. The factors $\Delta S_{d e p, n}$ and $\Delta S_{f a c, n}$ in $\Delta S_{n}^{\times}$are given by eqs. (57)(58). The depression and facilitation individual filters $X_{k, n}$ and $Z_{k, n}(k=1,2)$ are given by eqs. (53)-(55). These and the $\Delta S_{d e p, n}$ and $\Delta S_{f a c, n}$ filters were approximated by using the descriptive envelope model for STD in response to periodic presynaptic inputs (solid curves superimposed to the dotted curves) described in Section 3.3.2 by eqs. (32)-(34). The filter $\Delta S_{n}^{\times}$was approximated by using with eq. (43) with with $F$ and $G$ substituted by the corresponding approximations to $\Delta S_{d e p, n}$ and $\Delta S_{f a c, n}$. B. Dependence of the filter (global) time constants on the single events time constants. We used fixed values of $\tau_{d e p, 2}=\tau_{f a c, 2}$ and $\tau_{d e p, 1}$. Fig. 11 uses a different value of $\tau_{d e p, 1}$. The filter (global) time constants were computed using eq. (35). We used the following parameter values: $a_{d}=0.1, a_{f}=0.2, x_{\infty}=1, z_{\infty}=0, \tau_{d e p, 2}=\tau_{f a c, 2}=1000, \eta_{d e p}=\eta_{f a c}=0.5, \tau_{d e p, 1}=\tau_{f a c, 1}=10$, and $\Delta_{s p k}=10$. 
consistent with the presence of multiple depression and facilitation processes interacting as described by the cross (distribute) model.

\subsection{Persistence and disruption of temporal filters for $\Delta S_{n}=X_{n} Z_{n}$ in response to variable presynaptic spike trains}

By design, the temporal filters discussed above emerge in response to periodic presynaptic spike trains with period $\Delta_{s p k}$. Here we consider more realistic, irregular presynaptic spike trains with variable $(n-$ dependent) ISIs defined by the sequence $\left\{\Delta_{s p k, n}\right\}_{n=1}^{N_{s p k}}$. The natural candidates are Poisson spike trains (the ISI distribution follows a Poisson process with the parameter $r$ representing the mean firing rate) $[59,75]$. For Poisson spike trains both the mean ISI $(<I S I\rangle)$ and the standard deviation (SD) are equal to $r^{-1}$ and therefore the coefficient of variation $\mathrm{CV}=1$. For Poisson spike trains with absolute refractoriness $\left.I S I_{\min },<I S I\right\rangle=r^{-1}+I S I_{\min }$ and $\left.\mathrm{CV}=1-I S I_{\min }<I S I\right\rangle^{-1}$ [75], making them more regular. We use here $I S I_{\min }=1$ so that the irregularity remains high.

The presynaptic input spike-train variability causes variability in the output peak sequences $X_{n}, Z_{n}$ and the synaptic update $\Delta S_{n}=X_{n} Z_{n}$.

\subsubsection{Perturbations to periodic presynaptic spike train inputs}

To introduce some ideas, we consider perturbations of periodic presynaptic spiking patterns of the form

$$
\Delta_{s p k, n}=\Delta_{s p k}+\delta_{s p k, n}
$$

where $\Delta_{s p k}$ is constant ( $n$-independent) and $\delta_{p}=\left\{\delta_{s p k, n}\right\}_{n=1}^{N_{s p k}}$ is a sequence of real numbers. The exponential factors in (7)-(8) and (26) read

$$
e^{-\Delta_{s p k, n} / \tau_{s t p}}=e^{-\Delta_{s p k} / \tau_{s t p}} e^{-\delta_{s p k, n} / \tau_{s t p}},
$$

where $\tau_{s t p}$ represents $\tau_{d e p}$ or $\tau_{f a c}$.

If we further assume $\left|\delta_{s p k, n} / \tau_{s t p}\right| \ll 1$ for all $n$, then

$$
e^{-\Delta_{s p k, n} / \tau_{s t p}}=e^{-\Delta_{s p k} / \tau_{s t p}}\left[1-\frac{\delta_{s p k, n}}{\tau_{s t p}}+\mathcal{O}\left(\frac{\delta_{s p k, n}^{2}}{\tau_{s t p}^{2}}\right)\right] \sim e^{-\Delta_{s p k} / \tau_{s t p}}\left(1-\frac{\delta_{s p k, n}}{\tau_{s t p}}\right) .
$$

Eqs. (7)-(8) have the general form

$$
W_{n+1}=\alpha_{n} W_{n}+\beta_{n}
$$

for $n=1,2, \ldots, N_{s p k}-1$ with

$$
\begin{aligned}
& \alpha_{n, X}=\left(1-a_{d}\right) e^{-\Delta_{s p k, n} / \tau_{d e p}}, \\
& \beta_{n, X}=x_{\infty}\left(1-e^{-\Delta_{s p k, n} / \tau_{d e p}}\right), \\
& \alpha_{n, Z}=\left(1-a_{f}\right) e^{-\Delta_{s p k, n} / \tau_{f a c}},
\end{aligned}
$$

and

$$
\beta_{n, Z}=a_{f}+\left(1-a_{f}\right) z_{\infty}\left(1-e^{-\Delta_{s p k, n} / \tau_{f a c}}\right) .
$$

Substitution of (61) into these expressions yields 


$$
\begin{gathered}
\alpha_{n, X}=Q\left(a_{d}, \tau_{d e p}\right)-\left(1-a_{d}\right) e^{-\Delta_{s p k} / \tau_{d e p}} \frac{\delta_{s p k, n}}{\tau_{d e p}} \\
\beta_{n, X}=x_{\infty}\left(1-e^{-\Delta_{s p k} / \tau_{d e p}}\right)+x_{\infty} e^{-\Delta_{s p k} / \tau_{d e p}} \frac{\delta_{s p k, n}}{\tau_{d e p}} \\
\alpha_{n, Z}=Q\left(a_{f}, \tau_{f a c}\right)-\left(1-a_{f}\right) e^{-\Delta_{s p k} / \tau_{f a c}} \frac{\delta_{s p k, n}}{\tau_{f a c}}
\end{gathered}
$$

and

$$
\beta_{n, Z}=a_{f}+\left(1-a_{f}\right) z_{\infty}\left(1-e^{-\Delta_{s p k} / \tau_{f a c}}\right)+\left(1-a_{f}\right) z_{\infty} e^{-\Delta_{s p k} / \tau_{f a c}} \frac{\delta_{s p k, n}}{\tau_{f a c}} .
$$

The last terms in these expressions are the $\mathcal{O}\left(\delta_{s p k, n} / \tau_{s t p}\right)$ corrections to the corresponding parameters for the constant values of $\Delta_{s p k}\left(\delta_{p}=0\right.$, remaining terms) and contribute to the variance of the corresponding expressions. One important observation is that these variances monotonically increase with decreasing values of $\Delta_{s p k}$ (increasing values of $f_{s p k}$ ). A second important observation is the competing effects exerted by the parameters $\tau_{s t p}\left(\tau_{d e p}\right.$ and $\left.\tau_{f a c}\right)$ on the variance through the quotients

$$
e^{-\Delta_{s p k} / \tau_{s t p}} \frac{1}{\tau_{s t p}}
$$

As $\tau_{s t p}$ decreases (increases), $e^{-\Delta_{s p k} / \tau_{s t p}}$ decreases (increases) and $1 / \tau_{\text {stp }}$ increases (decreases). In the limit, $\tau_{s t p} \rightarrow 0, e^{-\Delta_{s p k} / \tau_{s t p}} \rightarrow 0$ and $1 / \tau_{s t p} \rightarrow \infty$ and vice versa. Therefore, one expects the variability to change non-monotonically with $\tau_{d e p}$ and $\tau_{f a c}$.

To proceed further, we use the notation

$$
\begin{array}{ll}
\alpha_{n, X}=\alpha_{X}+\delta_{\alpha, X, n}, & \beta_{n, X}=\beta_{X}+\delta_{\beta, X, n}, \\
\alpha_{n, Z}=\alpha_{Z}+\delta_{\alpha, Z, n} . & \beta_{n, Z}=\beta_{Z}+\delta_{\beta, Z, n} .
\end{array}
$$

Substituting into (87) in the Appendix A.2 we obtain

$X_{n}\left(\delta_{p}\right)=\bar{X}+\alpha_{X}^{n-1}\left(X_{1}-\bar{X}\right)+\alpha_{X}^{n-2} X_{1} \sum_{k=1}^{n-1} \delta_{\alpha, X, k}+\beta_{X} \sum_{k=1}^{n-1} \alpha_{X}^{n-k-2} \sum_{j=k+1}^{n-1} \delta_{\alpha, X, j}+\sum_{k=1}^{n-1} \alpha_{X}^{n-k-1} \delta_{\beta, X, k}$

and

$Z_{n}\left(\delta_{p}\right)=\bar{Z}+\alpha_{Z}^{n-1}\left(Z_{1}-\bar{Z}\right)+\alpha_{Z}^{n-2} Z_{1} \sum_{k=1}^{n-1} \delta_{\alpha, Z, k}+\beta_{Z} \sum_{k=1}^{n-1} \alpha_{Z}^{n-k-2} \sum_{j=k+1}^{n-1} \delta_{\alpha, Z, j}+\sum_{k=1}^{n-1} \alpha_{Z}^{n-k-1} \delta_{\beta, Z, k}$

where $\alpha_{X}=Q\left(a_{d}, \tau_{d e p}\right)$ and $\alpha_{Z}=Q\left(a_{f}, \tau_{f a c}\right)$. In (73) and (74) the first two terms correspond to the solution (24) and (25) to the corresponding systems in response to a presynaptic spike train input with a constant ISI $\Delta_{s p k}\left(\delta_{p}=0\right)$, which were analyzed in the previous sections. The remaining terms capture the (first order approximation) effects of the perturbations $\delta_{p}=\left\{\delta_{s p k, n}\right\}$, which depend on the model parameters through $\alpha_{X}, \beta_{X}, \alpha_{Z}, \beta_{Z}$ and the sequences $\delta_{\alpha, X, n}, \delta_{\beta, X, n}, \delta_{\alpha, Z, n}$ and $\delta_{\beta, Z, n}$ defined by the equations above.

These effects are accumulated as $n$ increases as indicated by the sums. However, as $n$ increases, both $Q\left(a_{d}, \tau_{d e p}\right)^{n}$ and $Q\left(a_{f}, \tau_{f a c}\right)^{n}$ decrease (they approach zero as $\left.n \rightarrow \infty\right)$ and therefore the effect of 
some terms will not be felt for large values of $n$ provided the corresponding infinite sums converge. On the other hand, the effect of the perturbations will be present as $n \rightarrow \infty$ in other sums. For example, for $k=n-1$ in the last sums in (73) and (74), $\alpha_{X}^{n-k-1}=\alpha_{Z}^{n-k-1}=1$ and therefore both $\delta_{\beta, X, n-1}$ and $\delta_{\beta, Z, n-1}$ will contribute $X_{n}$ and $Z_{n}$, respectively, for all values of $n$. For small enough values of $\delta_{p}$, the response sequences $X_{n}\left(\delta_{p}\right)$ and $Z_{n}\left(\delta_{p}\right)$ will remain close $X_{n}(0)$ and $Z_{n}(0)$, respectively (the response sequences to the corresponding unperturbed, periodic spike train inputs) and therefore the temporal filters will persist. Fig. 13 shows that this is also true for higher values of $\delta_{p}$. There, the sequence $\delta_{p}$ was normally distributed with zero mean and variance $D=1$. In all cases, the mean sequence values computed after the temporal filter decayed (by taking the second half of the sequence points for a total time $T_{\max }=100000, X_{c}$ and $Z_{c}$ ) coincides to a good degree of approximation with fixed-point of the unperturbed sequences $\bar{X}$ and $\bar{Z}$ (compared the corresponding solid and dotted curves).

A1

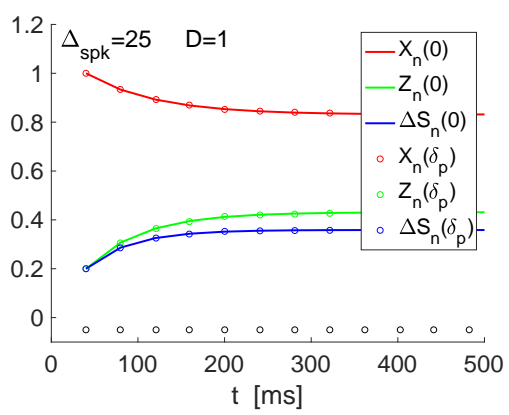

B1

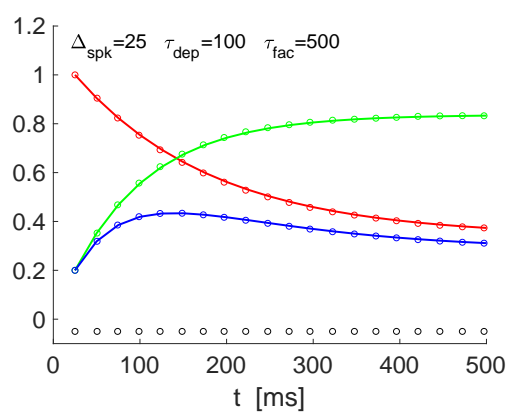

A2

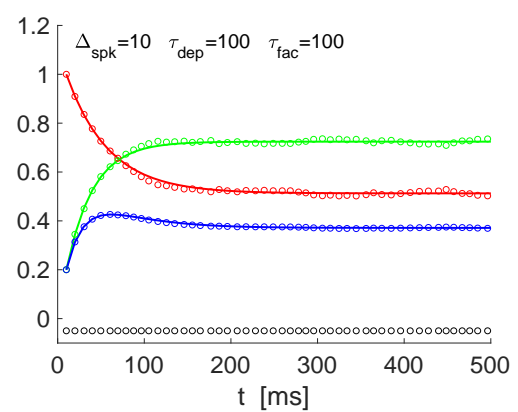

B2

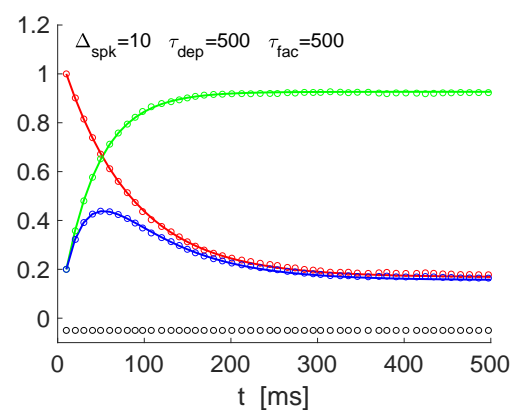

A3

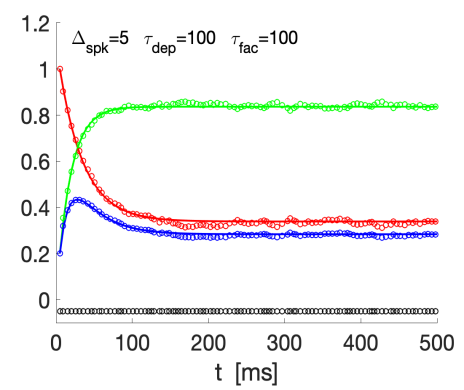

B3

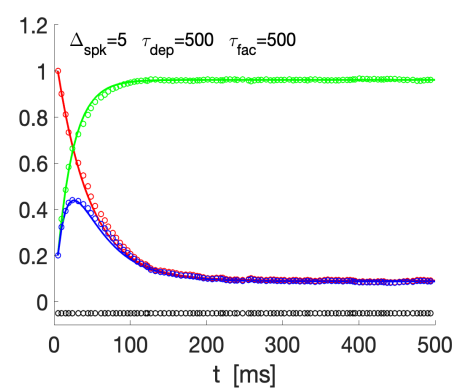

Figure 13: Temporal filters persist in response to variable presynaptic spike trains: Depression, facilitation and synaptic update response to normally distributed ISI perturbations to periodic spike train inputs. We used the recurrent equations (7) and (8) for $X_{n}$ and $Z_{n}$ respectively. The ISIs are perturbations around the ISI with constant $\Delta s p k$ according to eq. (59) where the sequence $\delta_{p}=\left\{\delta_{s p k, n}\right\}_{n=1}^{N_{s p k}}$ is normally distributed with zero mean and variance $D=1$. Simulations were run for a total time $T_{\max }=100000$. The sequences $X_{c}, Z_{c}$ and $\Delta S_{c}$ consist of the last half set of values for $X_{p}, Z_{p}$ and $\Delta S_{p}$, respectively, after the temporal filters decay to a vicinity of $\bar{X}, \bar{Z}$ and $\Delta_{S}^{-}$. A. $\tau_{d e p}=\tau_{f a c}=100$. A1. $\operatorname{var}(X c)=0.000015, \operatorname{var}(Z c)=0.000020$, $\operatorname{var}\left(\Delta S_{c}\right)=0.000002$. A2. $\operatorname{var}(X c)=0.000057, \operatorname{var}(Z c)=0.000065, \operatorname{var}\left(\Delta S_{c}\right)=0.000006 . \quad$ A3. $\operatorname{var}(X c)=0.000152$, $\operatorname{var}(Z c)=0.000092, \operatorname{var}\left(\Delta S_{c}\right)=0.000053$. B. $\tau_{d e p}=\tau_{f a c}=500$. B1. $\operatorname{var}(X c)=0.000006, \operatorname{var}(Z c)=0.000003, \operatorname{var}\left(\Delta S_{c}\right)=$ 0.000002. B2. $\operatorname{var}(X c)=0.000012, \operatorname{var}(Z c)=0.000005, \operatorname{var}\left(\Delta S_{c}\right)=0.000008$. B3. $\operatorname{var}(X c)=0.000016, \operatorname{var}(Z c)=0.000006$, $\operatorname{var}\left(\Delta S_{c}\right)=0.000013$. We used the following parameter values: $a_{d}=0.1, a_{f}=0.2, x_{\infty}=1, z_{\infty}=0$.

These results also confirm, by inspection, the previous theoretical observations. First, the variability is smaller for $\tau_{d e p}=\tau_{f a c}=500$ than for $\tau_{d e p}=\tau_{f a c}=100$. Second, as $\Delta_{s p k}$ decreases $f_{s p k}$ increases), the variability increases. For $\tau_{d e p}=\tau_{f a c}=100$ (Fig. 13-A) the variability of $\Delta S_{c}=X_{c} Z_{c}$ is smaller than 
the variabilities of both $X_{c}$ and $Z_{c}$. For $\tau_{d e p}=\tau_{f a c}=100$ (Fig. 13-B), the variability of $\Delta S_{c}=X_{c} Z_{c}$ is smaller than the variability of $X_{c}$, but not always smaller than the variability of $Z_{c}$.

While this approach is useful to understand certain aspects of the temporal synaptic update filtering properties in response to non-periodic presynaptic spike train inputs, it is limited since it does not admit arbitrarily large perturbations, which could cause the perturbed ISI to be negative. One solution is to make phase-based perturbations instead of time-based perturbations. But it is not clear whether comparison among patterns corresponding to different $\Delta_{s p k}$ are meaningful.

\subsubsection{Poisson distributed presynaptic spike train inputs}

Fig. 14 shows the initial response of the depression $\left(X_{n}\right)$, facilitation $\left(Z_{n}\right)$ and synaptic update peak $\left(\Delta S_{n}\right)$ sequences to Poisson distributed presynaptic spike train inputs for representative values of the spiking mean rate $r_{s p k}$ and the depression and facilitation time constants $\tau_{d e p}$ and $\tau_{f a c}$. A comparison between these responses and the temporal filters in response to periodic presynaptic spike inputs with a frequency equal to $r_{s p k}$ qualitatively shows that the temporal filtering properties tend to persist with different levels of fidelity. In Figs. 14-B2 and -C2, the temporal band pass-filter is terminated earlier than the corresponding deterministic one. In Fig. 14-B1, the temporal band-pass filter is initiated earlier than the corresponding deterministic one.

A

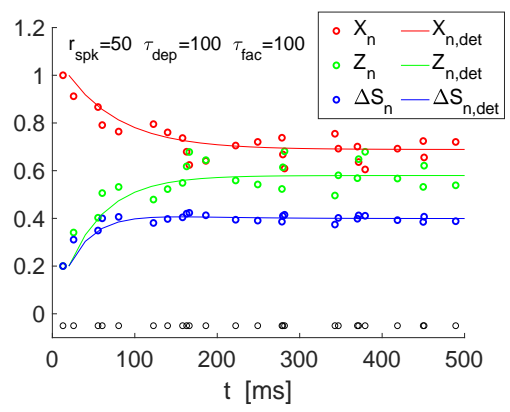

C1

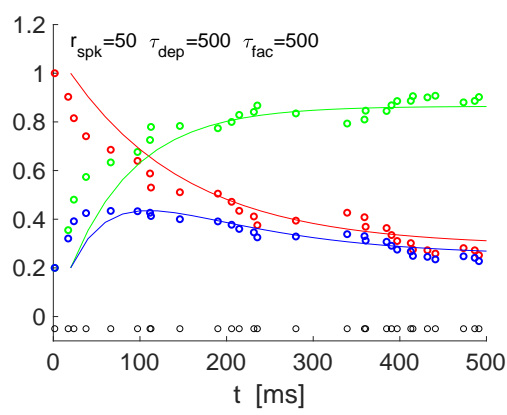

B1

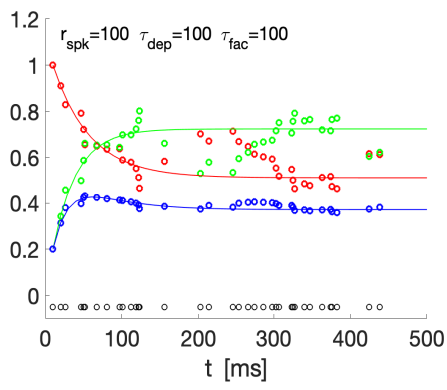

C2

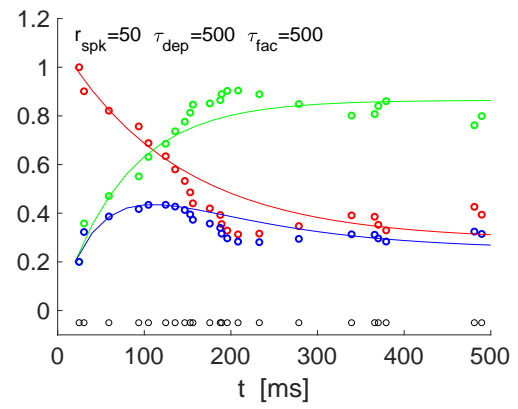

B2

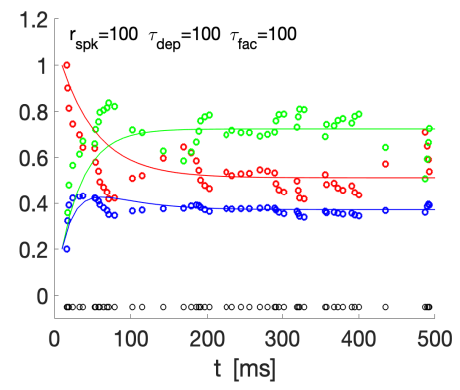

D

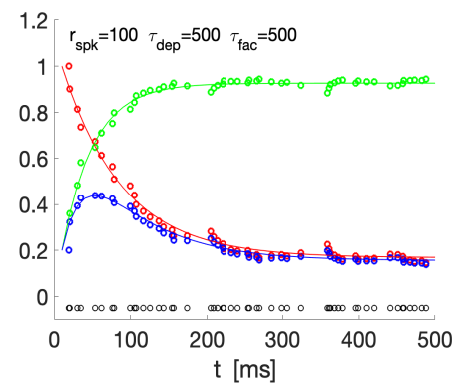

Figure 14: Temporal filters persist in response to variable presynaptic spike trains: Synaptic update response to Poisson distributed spike train inputs. We used the recurrent equations (7) and (8) for $X_{n}$ and $Z_{n}$ respectively. The ISIs have mean and standard deviation equal to $r_{s p k}$. Simulations were run for a total time $T_{\max }=200000(\Delta t=0.01)$. A, B. $\tau_{d e p}=\tau_{f a c}=100$.
A. $f_{s p k}=50$.
B. $f_{s p k}=100$. C, D. $\tau_{d e p}=\tau_{f a c}=500$.
C. $f_{s p k}=50$.
D. $f_{s p k}=100$
. We used the following parameter values: $a_{d}=0.1, a_{f}=0.2, x_{\infty}=1, z_{\infty}=0$.

In Fig. 15 we briefly analyze the response variability of the $X_{n}, Z_{n}$ and $\Delta S_{n}$ sequences induced by the presynaptic ISI variability. For $X_{n}$ and $Z_{n}$, the variability decreases with increasing values of $\tau_{d e p}$ 
and $\tau_{f a c}$ in an $r_{s p k}$-dependent manner (Fig. 15-A). For most cases, the variability also decreases with increasing values of $r_{s p k}$ in a $\tau_{d e p}$ - and $\tau_{f a c}$-manner (Fig. 15-B). An exception to this rule is shown in Fig. 15-B1 for the lower values of $r_{s p k}$.

A1

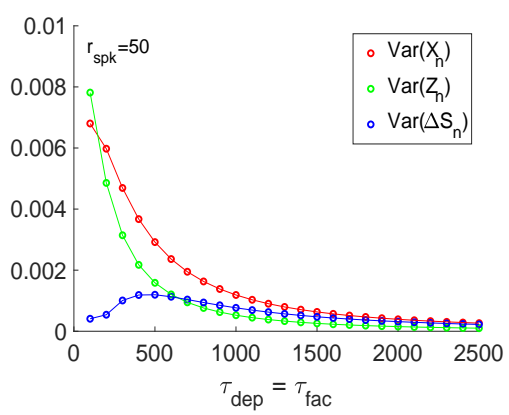

B1

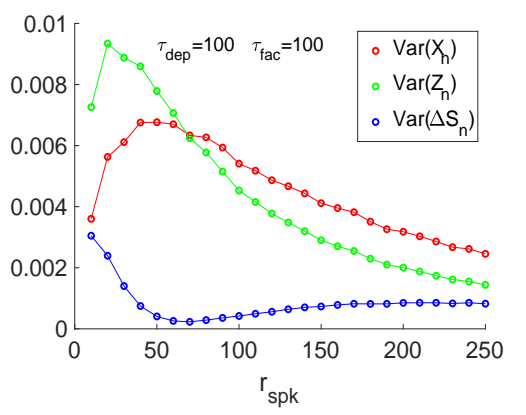

A2

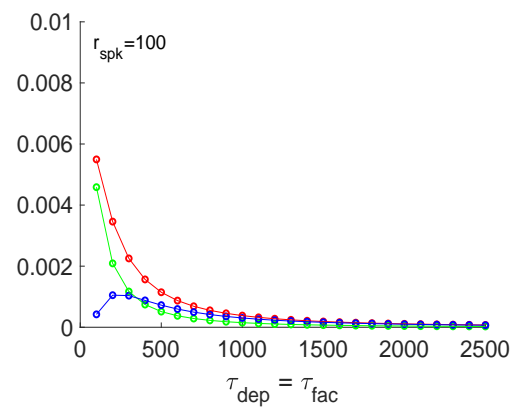

B2

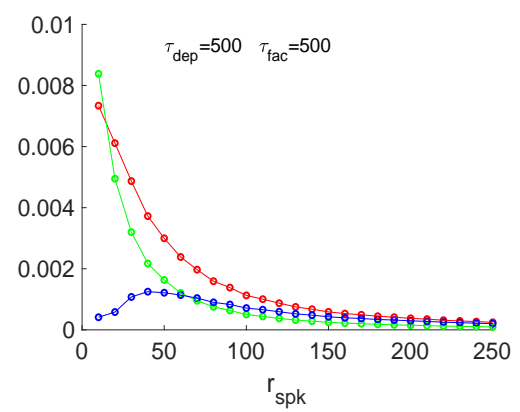

A3

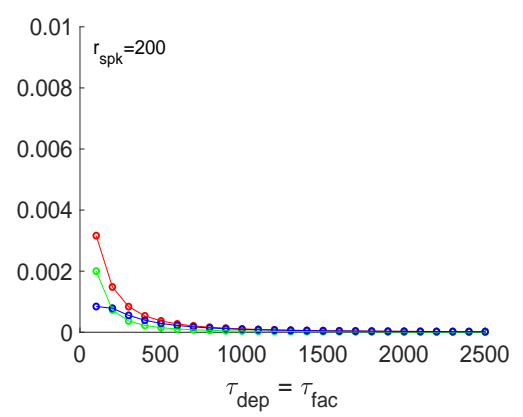

B23

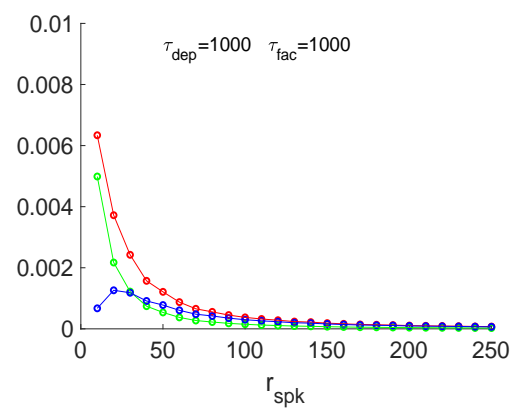

Figure 15: Response variability to non-periodic presynaptic spike trains: depression, facilitation and synaptic update response to Poisson spike train inputs. We used the recurrent equations (7) and (8) for $X_{n}$ and $Z_{n}$ respectively. The ISIs have mean and standard deviation equal to $r_{s p k}$. Simulations were run for a total time $T_{\max }=500000(\Delta t=0.01)$. A. Variance as a function of $\tau_{d e p}$ and $\tau_{f a c}\left(\tau_{d e p}=\tau_{f a c}\right)$ for fixed values of the Poisson input spike rate. A1. $r_{s p k}=50$. A2. $r_{s p k}=100$. A3. $r_{s p k}=200$. B. Variance as a function of the Poisson spike rate for fixed values of $\tau_{d e p}$ and $\tau_{f a c}\left(\tau_{d e p}=\tau_{f a c}\right)$. B1. $\tau_{d e p}=\tau_{f a c}=100$. B2. $\tau_{d e p}=\tau_{f a c}=500$. B3. $\tau_{d e p}=\tau_{f a c}=1000$. We used the following parameter values: $a_{d}=0.1$, $a_{f}=0.2, x_{\infty}=1, z_{\infty}=0$.

The variability of the $\Delta S_{n}$ sequences is more complex. Figs. 15-A1 and -A2 show examples of the peaking at intermediate values of $\tau_{d e p}$ and $\tau_{f a c}$. Fig. 15-B1 shows an example of the variability reaching a trough for an intermediate value of $r_{s p k}$ and relatively low values of $\tau_{d e p}$ and $\tau_{f a c}$, while Figs. 15-B2 and -B3 show examples of the variability peaking at intermediate values of $r_{s p k}$ for higher values of $\tau_{d e p}$ and $\tau_{f a c}$. This different dependence of the variability with the model parameters and input rates emerge as the result of the different variability properties of the product of the sequences $X_{n}$ and $Z_{n}$. A more detailed understanding of these properties is beyond the scope of this paper.

\subsection{The MT model exhibits similar filtering properties as the DA model: Dynam- ics of the depression $(R)$ and facilitation $(u)$ variables and their interaction}

The main difference between the DA model (5)-(6) and the MT model (12)-(13) is the update of the depression variables ( $x$ in the DA model and $R$ in the MT model). Notation aside, the dynamics for depression and facilitation variables $x$ and $z$ in the DA model are completely independent both during 
the presynaptic ISI and the update. In the MT model, in contrast, while the dynamics of the depression and facilitation variables $R$ and $u$ are independent during the presynaptic ISI as well as the $u$-update, the $R$-update is dependent on $u^{+}$. As a result, the difference equations describing the peak sequence dynamics for $R_{n}$ and $u_{n}$ (14)-(15) (peak envelope responses to periodic presynaptic inputs) are not fully independent, but the equation for $R_{n}$ is forced by the sequence $u_{n}$, which is independent of $R_{n}$. For the DA model, the difference equations for $Z_{n}$ and $Z_{n}$ are independent (7)-(8). Naturally, the steady-states $(\bar{X}$ and $\bar{Z})$ in the DA model are independent, while in the MT model, the steady state $\bar{R}$ depends on the steady state $\bar{u}$. Here we show that despite these differences and the increased difficulty in the interpretation of the analytical solution for the MT model as compared to the DA model for the determination of the long-term depression and facilitation filter time constants, the two models describe similar dynamics.

Standard methods (see Appendix A) applied to these linear difference equations for $\Delta_{s p k, n}=\Delta_{s p k}$ (independent of $n$ ) yield

$$
R_{n}=e^{-(n-1) \Delta_{s p k} / \tau_{d e p}} \prod_{k=1}^{n-1}\left(1-u_{n+1}\right)+\left(1-e^{-\Delta_{s p k} / \tau_{d e p}}\right) \sum_{k=1}^{n-1} e^{-(n-k-1) \Delta_{s p k} / \tau_{d e p}} \prod_{j=k+1}^{n-1}\left(1-u_{j+1}\right)
$$

and

$$
u_{n}=\bar{u}+Q\left(U, \tau_{f a c}\right)^{n-1}\left(u_{1}-\bar{u}\right)
$$

where $Q\left(U, \tau_{f a c}\right)$ is given by (26).

Because of the complexity of (75) we are not able to use the approach described in Section 3.3.1 to compute the (long-term) history-dependent time scales $\sigma_{d e p}$ and $\sigma_{f a c}$ in terms of the single event time scales $\left(\tau_{d e p}\right.$ and $\tau_{f a c}$ ) given by eq. (28). Instead, we use the descriptive modeling approach described in Section (3.3.2) by eqs. (32)-(35) and Section 3.3.3.

\section{Discussion}

In this paper, we set out to understand the mechanisms of generation of low-, high- and band-pass temporal filters in response to presynaptic spike trains in the presence of short-term plasticity in a feedforward network consisting of a periodic presynaptic spike train input synaptically connected to a postsynaptic cell in the presence of STP. We carried out a systematic analysis of the dependence of long-term time scales of the emergent temporal filters in terms of the time scales of the participating network components (the depression and facilitation time constants $\tau_{d e p}, \tau_{f a c}$, the synaptic decay time constant $\tau_{d e c}$, the membrane time constant $\tau_{m}$ and the presynaptic ISI) using two biophysically plausible phenomenological models that have been widely used in the literature [21, 40,57-65]. In the DA model [59], the depression and facilitation variables evolve independently and their interaction occurs at the level of the synaptic update $\Delta S$. In the MT model [58], the evolution of the depression variable is affected by the facilitation variable, in addition for both to interact at the synaptic update level. While, as we showed, both are analytically solvable, the advantage of the simpler DA model is that one can extract the temporal filter time scales from the analytical solutions. For the MT model, we have developed the descriptive model developed for that purpose (see Supplementary Material Section for the more detailed analysis). This descriptive modeling approach can be used and extended to more complex models for which analytical solutions are not available. Our results make a number of predictions that can be experimentally tested both in vitro and in vivo using dynamic clamp [76-78] and optogenetics [79,80].

An important question we addressed in our work is how the single event time constants (e.g., $\tau_{d e p}$, $\left.\tau_{f a c}, \tau_{d e c}\right)$, which control the systems' dynamics during the ISIs, are communicated to the temporal filters. In other words, how the temporal filters' long-term time constants $\left(\sigma_{d e p}, \sigma_{f a c}, \sigma_{s u m}\right)$ depend on the singleevent time constants. For the simple synaptic model with one-dimensional linear dynamics for the variable synaptic variable $S$ and a constant update $\Delta S$ (independent of $S$ ), the single-event and temporal filter 
time constant coincide. For the slightly more complex models for depression and facilitation, which have also linear one-dimensional dynamics during the ISI, but the updates depend on the depression and facilitation variables, there is a departure of the temporal filter time constant from the single event time constant. The dependence between the two is more complex and involves the corresponding update parameters as we showed analytically for the DA model and using the descriptive approach for the MT model. This reflects the dependence of the stationary properties of the temporal filters with the single event parameters.

Temporal band-pass filters are expected out of the multiplicative interaction between a low- and a high-pass filter under certain conditions, as we illustrated by using the descriptive model consisting of exponential functions decaying towards prescribed constant values. Similar to the depression and facilitation temporal filters, which are generated by one-dimensional linear difference equations, the band-pass filters can be considered as an overshoot type of solution to a linear difference equation. Overshoots are not possible for one-dimensional linear difference equations, but they are for two-dimensional linear difference equations, implying that two time scales would be enough to explain the properties of the band-pass filter. However, our results indicate that a third time scale, combining the first two, is needed to explain the band-pass filter properties.

The interaction between the depression/facilitation temporal filters with the synaptic and postsynaptic dynamics via de synaptic update generates additional low-, high- and band-pass temporal filters with additional time scales. For relatively low membrane time constants, the postsynaptic dynamics reflected the synaptic dynamics, but for higher membrane time constants, the postsynaptic filters departed from the synaptic ones, exhibiting temporal band-pass filters in the presence of synaptic temporal low-pass filters or in the presence of synaptic temporal band-pass filters, but peaking at different times.

In order to account for more realistic situations, we considered scenarios where more than one depression and facilitation processes with different time constants interact and scenarios where the presynaptic inputs are no longer periodic, but exhibit different types of variability including bursting and Poissondistributed spike trains. The results are consistent with the previous ones in the paper. However, more research is needed to investigate these scenarios in more detail and, in particular, to determine whether and under what circumstances the responses to variable input spiking patterns can be understood in terms of the results for periodic input spiking patterns.

While biophysically plausible, the phenomenological models of STP we used in this paper are relatively simple and leave out a number of important biological details that might contribute to determining the properties of STP-dependent temporal filters and their consequences for information processes. Further research is needed to understand the properties of these filters and how they emerge as the result of the interaction of the building blocks. An additional aspect that requires attention is the possible effect of astrocyte regulation of STP $[10,11]$ on the mechanisms of generation of STP-dependent temporal filters. Our work leaves out the mechanisms of generation and properties of the stationary low-, high- and band-pass filters and the associated phenomenon of synaptic and postsynaptic resonance. This will be discussed elsewhere.

The conceptual framework we developed in this paper allows developing ideas on the mechanisms of generation and properties of postsynaptic temporal filters in response to presynaptic inputs in the presence of STP. An important aspect of this framework is the separation of the feedforward network into a number of building blocks, each one with its own dynamics. The emerging temporal filters can be analyzed in terms of the hierarchical interaction of these building blocks. This conceptual framework can be used to investigate the properties of low-, high- and band-pass stationary, frequency-dependent filters and the emergence of synaptic and postsynaptic resonances, and it can be extended to include a number of more complex scenarios, including non-periodic synaptic spike trains (e.g., Poisson spike inputs, bursting patterns with two or more spiking frequencies), more complex networks (e.g., two recurrently connected cells with STP in both synapses, three-cell feedforward networks with STP in both synapses), the modulatory effects of astrocytes, more complex postsynaptic dynamics involving ionic currents that have been shown to produce resonances [81-86], and the generation of postsynaptic spiking temporal filters. 


\section{Acknowledgments}

This work was partially supported by the National Science Foundation grant DMS-1608077 (HGR) and an NSF Graduate Research Fellowship (YM). The authors are grateful to Allen Tannenbaum for useful comments and support, and to Farzan Nadim, Dirk Bucher and Nelly Daur for useful discussions.

\section{References}

[1] R. S. Zucker. Short-term synaptic plasticity. Annu. Rev. Neurosci., 12:13-31, 1989.

[2] R. S. Zucker and W. G. Regehr. Short-term synaptic plasticity. Annu. Rev. Physiol., 64:355-405, 2002.

[3] C. Stevens and Y. Wang. Facilitation and depression at single central synapses. Neuron, 14:795802, 1995.

[4] D. O. Hebb. The Organization of Behavior: A neuropsychological theory. Wiley, New York, 1949.

[5] T. V. Bliss and A. R. Gardner-Medwin. Long-lasting potentiation of synaptic transmission in the dentate area of the unanaestetized rabbit following stimulation of the perforant path. J. Physiol., 232:357-374, 1973.

[6] S. J. Martin, P. D. Grimwood, and R. G. Morris. Synaptic plasticity and memory: an evaluation of the hypothesis. Annu. Rev. Neurosci., 23:649-711, 2000.

[7] G. G. Turrigiano and S. B. Nelson. Homeostatic plasticity in the developing nervous system. Nat. Rev. Neurosci., 5:97-107, 2004.

[8] E. Marder and V. Thirumalai. Cellular, synaptic and network effects of neuromodulation. Neural Networks, 15:479-493, 2002.

[9] E. Marder. Neuromodulation of neuronal circuits : Back to the future. Neuron, 76:1-11, 2012.

[10] M. De Pitta, V. Volman, H. Berry, V. Parpura, A. Volterra, and E. Ben-Jacob. Computational quest for understanding the role of astrocyte signaling in synaptic transmission and plasticity. Front. Comp. Neurosci., 6:98, 2012.

[11] M. De Pitta, V. Volman, H. Berry, and E. Ben-Jacob. A tale of two stories: Astrocyte regulation of synaptic depression and facilitation. PLoS Comp. Biol., 7:e1002293, 2011.

[12] E. Fortune and G. Rose. Short-term plasticity as a temporal filter. Trends Neurosci., 24:381-385, 2001.

[13] D. Fioravante and W. G. Regehr. Short-term forms of presynaptic plasticity. Curr. Opin. Neurobiol., 21:260-274, 2011.

[14] A. Destexhe and E. Marder. Plasticity in single neuron and circuit computations. Nature, 431:785795, 2004.

[15] L. Abbott and W. G. Regehr. Synaptic computation. Nature, 431:796-803, 2004.

[16] A. Maass, W. Zador. Dynamic stochastic synapses as computational units. Neural Comput., 11:903917, 1999.

[17] J. F. Mejias and J. J. Torres. Maximum memory capacity on neural networks with short-term synaptic depression and facilitation. Neural Comput., 21:851-871, 2009.

[18] P.-Y. Deng and A. Klyachko. The diverse functions of short-term plasticity components in synaptic computations. Commun. Integr. Biol., 4:543-548, 2011.

[19] J. S. Dittman, A. C. Kreitzer, and W. G. Regehr. Interplay between facilitation, depression, and residual calcium at three presynaptic terminals. J. Neurosci., 20:1374-1385, 2000. 
[20] G. Silberberg, C. Wu, and H. Markram. Synaptic dynamics control the timing of neuronal excitation in the activated neocortical microcircuit. J. Physiol., 556:19-27, 2004.

[21] H. Markram, A. Gupta, A. andn Uziel, Y. Wang, and M. Tsodyks. Information processing with frequency-dependent synaptic connections. Neurobiology of Learning and Memory, 70:101-112, 1998.

[22] E. Fortune and G. Rose. Short-term synaptic plasticity contributes to the temporal filtering of electrosensory information. J. Neurosci., 20:7122-7130, 2000.

[23] E. Fortune and G. Rose. Passive and active membrane properties contribute to the temporal filtering properties of midbrain neurons in vivo. J. Neurosci., 17:3815-3825, 1997.

[24] E. Fortune and G. Rose. Temporal filtering properties of ampullary electrosensory neurons in the torus semicircularis of eigenmannia: evolutionary and computational implications. Brain Behav Evol, 49:312-323, 1997.

[25] E. Fortune and G. Rose. Roles of short-term plasticity in behavior. J Physiol Paris, 96:539-545, 2002.

[26] A. Thomson. Presynaptic frequency- and pattern-dependent filtering. J. Comp. Neurosci., 15:159202, 2003.

[27] M. S. Goldman, P. Maldonado, and L. F. Abbott. Redundancy reduction and sustained firing with stochastic depressing synapses. J. Neurosci., 22:584-591, 2002.

[28] J. F. Mejias and J. J. Torres. The role of synaptic facilitation in spike coincidence detection. J. Comp. Neurosci., 24:222-234, 2008.

[29] M. A. Bourjaily and P. Miller. Dynamic afferent synapses to decision-making networks improve performance in tasks requiring stimulus associations and discriminations. J. Neurophysiol., 108:513-527, 2012.

[30] V. A. Klyachko and C. F. Stevens. Excitatory and feed-forward inhibitory hippocampal synapses work synergistically as an adaptive filter of natural spike trains. PLoS Comp. Biol., 4:e207, 2006.

[31] A. A. George, A. M. Lyons-Warren, X. Ma, and B. A. Carlson. A diversity of synaptic filters are created by temporal summation of excitation and inhibition. J. Neurosci., 31:14721-14734, 2011.

[32] J. E. Lewis and L. Maler. Dynamics of electrosensory feedback: short-term plasticity and inhibition in a parallel fiber pathway. J. Neurophysiol., 88:1695-1702, 2002.

[33] U. Kandaswamy, P.-Y. Deng, C. Stevens, and V. A. Klyachko. The role of presynaptic dynamics in processing of natural spike trains in hippocampal synapses. J. Neurosci., 30:15904-15914, 2010.

[34] J. A. Varela, K. Sen, J. Gibson, J. Fost, L. F. Abbott, and S. B. Nelson. A quantitative description of short-term plasticity at excitatory synapses in layer $2 / 3$ of rat primary visual cortex. J. Neurosci., 17:7926-7940, 1997.

[35] F. S. Chance, S. B. Nelson, and L. F. Abbott. Synaptic depression and the temporal response characteristics of v1 cells. J. Neurosci., 18:4785-4799, 1998.

[36] A. Zador and L. Dobrunz. Dynamic synapses in the cortex. Neuron, 19:1-4, 1997.

[37] J. E. Lisman. Bursts as a unit of neural information: making unreliable synapses reliable. Trends Neurosci., 20:38-43, 1997.

[38] E. M. Izhikevich, N. S. Desai, E. C. Walcott, and F. C. Hoppensteadt. Bursts as a unit of neural information: Selective communication via resonance. Trends Neurosci., 26:161-167, 2003.

[39] M. Tsodyks and H. Markram. Plasticity of neocortical synapses enables transitions between rate and temporal coding. Lect. Notes Comput. Sci, 1112:445-450, 1996.

[40] M. Tsodyks and H. Markram. The neural code between neocortical pyramidal neurons depends on neurotransmitter release probability. Proc. Natl. Acad. Sci. USA, 94:719-723, 1997. 
[41] Z. Rotman, P.-Y. Deng, and V. A. Klyachko. Short-term plasticity optimizes synaptic information transmission. J. Neurosci., 31:14800-14809, 2011.

[42] L. F. Abbott, J. A. Varela, K. Sen, and S. B. Nelson. Synaptic depression and cortical gain control. Science, 275:220-224, 1997.

[43] M. Tsodyks and S. Wu. Short-term synaptic plasticity. Scholarpedia, 8:3153, 2013.

[44] G. Fuhrmann, I. Segev, and H. Markram. Coding of temporal information by activity-dependent synapses. J. Physiol., 556:19-27, 2004.

[45] UI R. Karmarkar and D. V. Buonomano. Timing in the absence of clocks: Encoding time in neural network states. Neuron, 53:427-438, 2007.

[46] D. Buonomano and W. Maass. State-dependent computations: spatiotemporal processing in cortical networks. Nat. Rev. Neurosci., 10:113-125, 2009.

[47] G. Mongillo, O. Barak, and M. Tsodyks. Synaptic theory of working memory. Science, 319:15431546, 2015.

[48] A. Loebel and M. Tsodyks. Computation by ensemble synchronization in recurrent networks with synaptic depression. J. Comp. Neurosci., 13:111-124, 2002.

[49] O. Barak and M. Tsodyks. Persistent activity in neural networks with dynamic synapses. PLoS Comp. Biol., 3:e35, 2007.

[50] D. L. Cook, P. C. Schwindt, L. A. Grande, and W. J. Spain. Synaptic depression in the localization of sound. Nature, 421:66-70, 2003.

[51] M. H. Hennig, M. Postlethwaite, I. D. Forsythe, and B. P. Graham. Interactions between multiple sources of short-term plasticity during evoked and spontaneous activity at the rat calyx of held. $\mathrm{J}$. Physiol., 586:3129-3146, 2008.

[52] S. Carver, E. Roth, N. J. Cowan, and E. S. Fortune. Synaptic plasticity can produce and enhance direction selectivity. PLoS Comp. Biol., 4:e32, 2008.

[53] S.-I. Amari. Dynamics of pattern formation in lateral-inhibition type neural fields. Biol. Cybern., 27:77-87, 1977.

[54] W. Yuan, O. Dimigen, W. Sommer, and C. Zhou. A model of microsaccade-related neural responses induced by short-term depression in thalamocortical synapses. Front. Comp. Neurosci., 7:47, 2013.

[55] O. Barak, M. Tsodyks, and R. Romo. Neuronal population coding of parametric working memory. J. Neurosci., 319:1543-1546, 2008.

[56] G. Deco, E. Rolls, and R. Romo. Synaptic dynamics and decision making. Proc. Natl. Acad. Sci. USA, 107:7547-7549, 2010.

[57] M. Tsodyks, K. Pawelzik, and H. Markram. Neural networks with dynamic synapses. Neural Comput., 10:821-835, 1998.

[58] H. Markram, Y. Wang, and M. Tsodyks. Differential signaling via the same axon of neocortical pyramidal neurons. Proc. Natl. Acad. Sci. USA, 95:5323-5328, 1998.

[59] P. Dayan and L. F. Abbott. Theoretical Neuroscience. The MIT Press, Cambridge, Massachusetts, 2001.

[60] B. Lindner, D. Gangloff, A. Longtin, and J. E. Lewis. Broadband coding with dynamic synapses. J. Neurosci., 29:2076-2088, 2009.

[61] J. A. Varela, S. Song, G. G. Turrigiano, and S. B. Nelson. Differential depression at excitatory and inhibitory synapses in visual cortex. J. Neurosci., 19:4293-4304, 1999.

[62] A. Morrison, M. Diesmann, and W. Gerstner. Phenomenological models of synaptic plasticity based on spike timing. Biol. Cybern., 98:459-478, 2008. 
[63] H. Markram, D. Pikus, A. Gupta, and M. Tsodyks. Potential for multiple mechanisms, phenomena and algorithms for synaptic plasticity at single synapses. Neuropharmacology, 37:489-500, 1998.

[64] J. D. Drover, V. Tohidi, A. Bose, and F. Nadim. Combining synaptic and cellular resonance in a feedforward neuronal network. Neurocomputing, 70:2041-2045, 2007.

[65] M. Tsodyks, A. Uziel, and H. Markram. Synchrony generation in recurrent networks with frequencydependent synapses. J. Neurosci., 20:1-5, 2000.

[66] G. B. Ermentrout and D. Terman. Mathematical Foundations of Neuroscience. Springer, 2010.

[67] H. G. Rotstein and E. G. Tabak. Analysis of spike-driven processes through attributable components. Communications in Mathematical Sciences), 17:1177-1192, 2019.

[68] K. L. Magleby and J. E. Zengel. A quantitative description of stimulation-induced changes in transmitter release at the frog neuromuscular junction. J. Gen. Physiol., 80:613-638, 1982.

[69] M. H. Hennig. Theoretical models of synaptic short term plasticity. Front. Comp. Neurosci., 7:45, 2013.

[70] H. Markram and M. Tsodyks. Redistribution of synaptic efficacy between neocortical pyramidal neurons. Nature, 382:807-810, 1996.

[71] R. L. Burden and J. D. Faires. Numerical analysis. PWS Publishing Company - Boston, 1980.

[72] A. Destexhe, Z. F. Mainen, and T. Sejnowski. Kinetic models of synaptic transmission. In Methods in Neural Modeling. Koch, C. and Segev, I., editors, second edition. MIT Press: Cambridge, Massachusetts, pages 1-25, 1998.

[73] A. Destexhe, Z. F. Mainen, and T. Sejnowski. An efficient method for computing synaptic conductances based on a kinetic model of receptor binding. Neural Comput., 6:14-18, 1994.

[74] R. Latorre, J. J. Torres, and P. Varona. Interplay between subthreshold oscillations and depressing synapses in single neurons. PLOS ONE, 11:e0145830, 2016.

[75] W. Gerstner, W. M. Kistler, R. Naud, and L. Paninski. Neuronal dynamics: From single neurons to networks and models of cognition. Cambridge University Press, 2014.

[76] A. A. Sharp, M. B. O'Neil, L. F. Abbott, and E. Marder. The dynamic clamp: artificial conductances in biological neurons. Trends Neurosci., 16:389-394, 1993.

[77] A. A. Sharp, M. B. O'Neil, L. F. Abbott, and E. Marder. Dynamic clamp: computer-generated conductances in real neurons. J. Neurophysiol., 69:992-995, 1993.

[78] A. A. Prinz, L. F. Abbott, and E. Marder. The dynamic clamp comes of age. Trends Neurosci., 27:218-224, 2004.

[79] F. Zhang, V. Gradinaru, A. R. Adamantidis, R. Durand, R. D. Airan, L. de Lecea, and K. Deisseroth. Optogenetic interrogation of neural circuits: Technology for probing mammalian brain structures. Nature Protocols, 5:439-456, 2010.

[80] J. G. Bernstein and E. S. Boyden. Optogenetic tools for analyzing the neural circuits of behavior. Curr. Opin. Neurobiol., 22:61-71, 2012.

[81] M. J. E. Richardson, N. Brunel, and V. Hakim. From subthreshold to firing-rate resonance. J. Neurophysiol., 89:2538-2554, 2003.

[82] H. G. Rotstein and F. Nadim. Frequency preference in two-dimensional neural models: a linear analysis of the interaction between resonant and amplifying currents. J. Comp. Neurosci., 37:9-28, 2014.

[83] B. Hutcheon and Y. Yarom. Resonance, oscillations and the intrinsic frequency preferences in neurons. Trends Neurosci., 23:216-222, 2000. 
[84] H. G. Rotstein. Subthreshold amplitude and phase resonance in models of quadratic type: nonlinear effects generated by the interplay of resonant and amplifying currents. J. Comp. Neurosci., 38:325354, 2015.

[85] H. Hu, K. Vervaeke, and J. F. Storm. Two forms of electrical resonance at theta frequencies generated by M-current, h-current and persistent $\mathrm{Na}^{+}$current in rat hippocampal pyramidal cells. J. Physiol., 545.3:783-805, 2002.

[86] B. Hutcheon, R. M. Miura, and E. Puil. Subthreshold membrane resonance in neocortical neurons. J. Neurophysiol., 76:683-697, 1996.

[87] J. Dudel and S. W. Kuffler. Presynaptic inhibition at the crayfish neuromuscular junction. J. Physiol., 155:543-562, 1961.

[88] J. D. Clements, R. A. Lester, G. Tong, C. E. Jahr, and G. L. Westbrook. The time course of glutamate in the synaptic cleft. Science, 258:1498-1501, 1992.

[89] D. Colquhoun, P. Jonas, and B. Sakmann. Action of brief pulses of glutamate on AMPA/kainate receptors in patches from different neurones of rat hippocampal slices. J. Physiol., 458:261-287, 1992.

[90] Y. Manor and F. Nadim. Synaptic depression mediates bistability in neuronal networks with recurrent inhibitory connectivity. J. Neurosci., 21:9460-9470, 2001.

[91] Y. Manor, F. Nadim, L. Abbott, and E. Marder. Temporal dynamics of graded synaptic transmission in the lobster stomatogastric ganglion. J. Neurosci., 17:5610-5621, 1997.

\section{A 1D linear difference equations}

\section{A.1 Constant coefficients}

Consider the following linear difference equation

$$
w_{n+1}=\alpha w_{n}+\beta, \quad n=1,2, \ldots
$$

where $\alpha$ and $\beta$ are constants. The steady-state for this equation, if it exists, is given by

$$
\bar{w}=\frac{\beta}{1-\alpha} .
$$

By solving (77) recurrently and using

$$
\sum_{n=0}^{N} a^{n}=\frac{a^{N+1}-1}{a-1}
$$

where $a \neq 1$ is a real number, one gets

$$
w_{n}=\alpha^{n-1} w_{1}+\beta \frac{\alpha^{n-1}-1}{\alpha-1} .
$$

Substitution of (78) into this equation yields

$$
w_{n}=\bar{w}+\alpha^{n-1}\left(w_{1}-\bar{w}\right) .
$$

Application of formula (81) to the difference equations (7) and (8) gives, respectively,

$$
X_{n}=\bar{X}+(1-\bar{X})\left(1-a_{d}\right)^{n-1} e^{-(n-1) \Delta_{s p k} / \tau_{d e p}}=
$$




$$
\bar{X}+(1-\bar{X}) e^{-(n-1)\left[\Delta_{s p k} / \tau_{d e p}-\ln \left(1-a_{d}\right)\right]}
$$

and

$$
\begin{gathered}
Z_{n}=\bar{Z}+(1-\bar{Z})\left(1-a_{f}\right)^{n-1} e^{-(n-1) \Delta_{s p k} / \tau_{f a c}}= \\
\bar{Z}+(1-\bar{Z}) e^{-(n-1)\left[\Delta_{s p k} / \tau_{f a c}-\ln \left(1-a_{f}\right)\right]} .
\end{gathered}
$$

\section{A.2 Variable ( $n$-dependent) coefficients}

Consider the following linear difference equation

$$
w_{n+1}=\alpha_{n} w_{n}+\beta_{n}, \quad n=1,2, \ldots
$$

By solving (77) recurrently one gets

$$
w_{n}=\left(\prod_{k=1}^{n-1} \alpha_{k}\right) x_{1}+\sum_{k=1}^{n-1}\left(\prod_{j=k+1}^{n-1} \alpha_{j}\right) \beta_{k}
$$

where we are using the convention $\prod_{j_{1}}^{j_{2}}=1$ if $j_{1}>j_{2}$. Eq. (85) reduces to eq. (83) if both coefficients in (85) are constant.

Consider now eq. (84) where the coefficients are expressed as small perturbations $\delta_{\alpha, n} \ll 1$ and $\delta_{\beta, n} \ll 1(n=1,2, \ldots)$, respectively, of constant coefficients

$$
\alpha_{n}=\alpha+\delta_{\alpha, n} \quad \text { and } \quad \beta_{n}=\beta+\delta_{\beta, n} .
$$

To the first order approximation, the solution (85) reads

$$
\begin{aligned}
w_{n} & =\alpha^{n-1} w_{1}+\beta \frac{\alpha^{n-1}-1}{\alpha-1}+\alpha^{n-2} w_{1} \sum_{k=1}^{n-1} \delta_{\alpha, k}+\beta \sum_{k=1}^{n-1} \alpha^{n-k-2} \sum_{j=k+1}^{n-1} \delta_{\alpha, j}+\sum_{k=1}^{n-1} \alpha^{n-k-1} \delta_{\beta, k}=. \\
& =\bar{w}+\alpha^{n-1}\left(w_{1}-\bar{w}\right)+\alpha^{n-2} w_{1} \sum_{k=1}^{n-1} \delta_{\alpha, k}+\beta \sum_{k=1}^{n-1} \alpha^{n-k-2} \sum_{j=k+1}^{n-1} \delta_{\alpha, j}+\sum_{k=1}^{n-1} \alpha^{n-k-1} \delta_{\beta, k} .
\end{aligned}
$$

\section{B Some properties of $\bar{X}$ and $\bar{Z}$ and their dependence with $\Delta_{s p k}$ and}

$\tau_{d e p / f a c}$

Consider $\bar{X}$ and $\bar{Z}$ given by (9) and (10), respectively.

\section{B.1 Monotonic dependence of $\bar{X}$ and $\bar{Z}$ with $\Delta_{s p k}$}

If $a_{d}>0$ and $x_{\infty}>0$, then $\bar{X}$ is an increasing function of $\Delta_{s p k}$ and a decreasing function of $f_{s p k}$. This results from

$$
\frac{\partial \bar{X}}{\partial \Delta_{s p k}}=\frac{x_{\infty} a_{d} e^{-\Delta_{s p k} / \tau_{d e p}}}{\tau_{d e p}\left[1-\left(1-a_{d}\right) e^{-\Delta_{s p k} / \tau_{d e p}}\right]^{2}}>0
$$


If $a_{f}<1$ and $z_{\infty}<1$, then $\bar{Z}$ is a decreasing function of $\Delta_{s p k}$ and an increasing function of $f_{s p k}$. This results from

$$
\frac{\partial \bar{Z}}{\partial \Delta_{s p k}}=\frac{a_{f}\left(1-a_{f}\right)\left(z_{\infty}-1\right)}{\tau_{f a c}\left[1-\left(1-a_{f}\right) e^{-\Delta_{s p k} / \tau_{f a c}}\right]^{2}}<0
$$

\section{B.2 Monotonic dependence of $\bar{X}$ and $\bar{Z}$ with $\tau_{\text {dep/fac }}$}

If $a_{d}>0$ and $x_{\infty}>0$, then $\bar{X}$ is an decreasing function of $\tau_{d e p}$. This results from

$$
\frac{\partial \bar{X}}{\partial \Delta_{s p k}}=-\frac{\Delta_{s p k} x_{\infty} a_{d} e^{-\Delta_{s p k} / \tau_{d e p}}}{\tau_{d e p}^{2}\left[1-\left(1-a_{d}\right) e^{-\Delta_{s p k} / \tau_{d e p}}\right]^{2}}<0
$$

If $a_{f}<1$ and $z_{\infty}<1$, then $\bar{Z}$ is a decreasing function of $\tau_{f a c}$. This results from

$$
\frac{\partial \bar{Z}}{\partial \Delta_{s p k}}=-\frac{\Delta_{s p k} a_{f}\left(1-a_{f}\right)\left(z_{\infty}-1\right)}{\tau_{f a c}^{2}\left[1-\left(1-a_{f}\right) e^{-\Delta_{s p k} / \tau_{f a c}}\right]^{2}}>0
$$

\section{Models of synaptic depression and facilitation}

\section{C.1 Depression - facilitation model used in [74]}

Following [72,73], the synaptic variables $S$ obey a kinetic equation of the form

$$
\frac{d S}{d t}=N(V) \frac{(1-S)}{\tau_{r}}-\frac{S}{\tau_{d}}
$$

where $N(V)(\mathrm{mM})$ representes the neurotransmitter concentration in the synaptic cleft. Neurotransmitters are assumed to be released quickly upon the arrival of a presynaptic spike and remain in the synaptic cleft for the duration of the spike ( $\sim 1 \mathrm{~ms})$. This can be modeled by either using a sigmoid function

$$
N(V)=\frac{1+\tanh (V / 4)}{2},
$$

or a step function if the release is assumed to be instantaneous. The parameters $\tau_{r}$ and $\tau_{d}$ are the rise and decay time constants respectively (msec).

This model assumes $N(V)$ is independent of the spiking history (the value of $N(V)$ during a spike is constant, except possibly for the dependence on $V$ ). (There is evidence that this is not realistic [70,87].) In [74], the "activated" time was $1 \mathrm{~ms}[88,89]$.

In [74], the follow the description of the synaptic short-term dynamics following $[40,57]$. They use $[T]$ instead of $N(V)$ where $[T]$ is constant during the release time and zero otherwise, and they define $[T]=\kappa \Delta S_{n}$. Effectively,

$$
\frac{d S}{d t}=\kappa \Delta S_{n} N(V) \frac{(1-S)}{\tau_{r}}-\frac{S}{\tau_{d}}
$$

\section{C.2 Depression model used in [90]}

Following experimental procedures described in [91], the synaptic current is described by $I_{s y n}=G_{e x} a d(V-$ $\left.E_{e x}\right)$ where $a$ and $d$ are variables that represent activation and depression processes, respectively. They follow the form:

$$
\frac{d y}{d t}=\frac{y_{\infty}\left(V_{\mathrm{pre}}-y\right)}{\tau_{y}}
$$


where $y=a, d$. The steady-state of $y$ is given by

$$
y_{\infty}=\frac{1}{1+\exp \left(\left(V-V_{x}\right) / k\right)},
$$

and its time constant follows

$$
\tau_{y}=\tau_{1}+\frac{\tau_{h}-\tau_{1}}{1+\exp \left(\left(V-V_{x}\right) / k\right)} .
$$

This model is used in [90] to describe bistability in pacemaker networks with recurrent inhibition and depressing synapses. Parameters in these equations are experimentally fitted from the pyloric network of the crab Cancer borealis.

\section{Additional model formulations for multiple depression-facilitation processes}

In Section 3.7 we discussed the model formulation (51)-(52) describing the interplay of two depressionfacilitations processes. A number of additional, simplified formulations are possible based on different assumptions. The models we propose here are natural mathematical extensions of the single depression/facilitation processes discussed in the main body of this paper. They are phenomenological models, not based on any experimental observation or theoretical foundation, and they are limited in their general applicability. However, they are useful to explore the possible scenarios underlying the interplay of multiple depression and facilitation time scales affecting the PSP dynamics of a cell in response to presynaptic input trains.

\section{D.1 Additive and multiplicative segregated-processes models}

In the additive and multiplicative segregated models, the variable $M$ is given, respectively, by

$$
M^{+}(t)=(1-\alpha) x_{1}(t) z_{1}(t)+\alpha x_{2}(t) z_{2}(t)
$$

and

$$
M^{*}(t)=\left[x_{1}(t) z_{1}(t)\right]^{1-\alpha}\left[x_{2}(t) z_{2}(t)\right]^{\alpha}
$$

where the parameter $\alpha \in[0,1]$ controls the relative contribution of each of the processes. Correspondingly, the updates are given by

$$
\Delta S_{n}^{+}=(1-\alpha) X_{1, n} Z_{1, n}+\alpha X_{2, n} Z_{2, n}
$$

and

$$
\Delta S_{n}^{*}=\left[X_{1, n} Z_{1, n}\right]^{1-\alpha}\left[X_{2, n} Z_{2, n}\right]^{\alpha} .
$$

For $\alpha=0, \Delta S_{n}^{+}$and $\Delta S_{n}^{*}$ reduce to $\Delta S_{1, n}$ (single depression-facilitation process). This accounts for the regimes where $\tau_{d e p, 2}, \tau_{f a c, 2} \ll 1$. If the two processes are equal $\left(\tau_{d e p, 1}=\tau_{d e p, 2}\right.$ and $\left.\tau_{f a c, 1}=\tau_{f a c, 2}\right)$, then $\Delta S_{n}^{+}$and $\Delta S_{n}^{*}$ also reduce to $\Delta S_{1, n}$. However, these models fail to account for the reducibility in the situations where only $\tau_{d e p, 2} \ll 1$ or $\tau_{f a c, 2} \ll 1$, but not both. The option of considering depression to be described by $x_{1}$ and facilitation by $z_{2}$ (with $\tau_{f a c, 1}, \tau_{d e p, 2} \ll 1$ ) is technically possible in the context of the model, but it wouldn't be consistent with the model description of single depression-facilitation processes, and it will make no sense to use the model in this way. In general, this model would be useful when the depression and facilitation time scales for each process 1 and 2 are comparable and the differences in these time scales across depression/facilitation processes should be large enough. 


\section{D.2 Fully multiplicative model}

One natural way to extend the variable $M$ to more than one process is by considering

$$
M^{\#}(t)=x_{1}(t) z_{1}(t) x_{2}(t) z_{2}(t)
$$

and the synaptic update, given by

$$
\Delta S_{n}^{\#}=X_{1, n} Z_{1, n} X_{2, n} Z_{2, n} .
$$

This formulation presents us with a number of consistency problems related to the reducibility (or lack of thereoff) to a single depression-facilitation process in some limiting cases when, for example, the two depression or facilitation time constants are very similar and therefore the associated processes are almost identical, or the depression or facilitation time constants are very small and therefore the envelopes of the associated processes are almost constant across cycles.

More specifically, first, if $\tau_{d e p, 2}, \tau_{f a c, 2} \ll 1$ (almost no STD), then $X_{2, n} Z_{2, n} \sim \bar{X}_{2} \bar{Z}_{2}=a_{f}$ for all $n$ after a very short transient and therefore $\Delta S_{n}^{\#}=X_{1} Z_{1} a_{f} \neq \Delta S_{1, n}$. One way, perhaps the simplest, to address this is to divide the expressions (102) and (103) by $a_{f}^{2}$ and redefine $\Delta S_{k, n}$ for the single depression-facilitation process accordingly. Specifically,

$$
\Delta S_{n}^{\#}=\frac{X_{1, n} Z_{1, n} X_{2, n} Z_{2, n}}{a_{f}^{2}}=\frac{X_{1, n} Z_{1, n}}{a_{f}} \frac{X_{2, n} Z_{2, n}}{a_{f}},
$$

where we use the notation

$$
\Delta S_{1, n}=\frac{X_{1, n} Z_{1, n}}{a_{f}} \quad \text { and } \quad \Delta S_{2, n}=\frac{X_{2, n} Z_{2, n}}{a_{f}} .
$$

The effect of redefining $\Delta S_{k, n}$ by dividing the original expression (used in the previous sections) does not affect the time constants and the differences in the values between the two formulations is absorbed by the maximal synaptic conductance.

Second, if $\tau_{d e p, 1}=\tau_{d e p, 2}$ and $\tau_{f a c, 1}=\tau_{f a c, 2}$, then $X_{1, n}=X_{2, n}$ and $Z_{1, n}=Z_{2, n}$ for all $n$, and $\Delta S_{n}^{\#}=\Delta S_{1, n}^{2}$ instead of $\Delta S_{n}^{\#}=\Delta S_{1, n}$. In order to address this, the synaptic update can be modified to

$$
\Delta S_{n}^{\#}=\left[X_{1, n} X_{2, n}\right]^{\lambda_{d e p}}\left[\frac{Z_{1, n} Z_{2, n}}{a_{f}^{2}}\right]^{\lambda_{f a c}}
$$

where

$$
\lambda_{d e p}=\frac{1}{H\left(\left|\tau_{d e p, 1}-\tau_{d e p, 2}\right|\right)} \quad \quad \text { and } \quad \lambda_{f a c}=\frac{1}{H\left(\left|\tau_{f a c, 1}-\tau_{f a c, 2}\right|\right)}
$$

and $H(\Delta \tau)$ is a rapidly decreasing function satisfying $H(0)=2$ and $\lim _{\Delta \tau \rightarrow \infty} H(\Delta \tau)=1$. In our simulations we will use

$$
H(\Delta \tau)=1+e^{-\Delta \tau / \beta}
$$

with $\beta>0$. Correspondingly,

$$
M^{\#}=\left[x_{1}(t) x_{2}(t)\right]^{\lambda_{d e p}}\left[\frac{z_{1}(t) z_{2}(t)}{a_{f}^{2}}\right]^{\lambda_{f a c}}
$$

In this way, 
- If $\tau_{d e p, 1}=\tau_{d e p, 2}$, then $X_{1, n}=X_{2, n}$ for all $n$ and $\lambda_{d e p}=1 / 2$. This gives

$$
\Delta S_{n}^{\#}=X_{1, n}\left[\frac{Z_{1, n} Z_{2, n}}{a_{f}^{2}}\right]^{\lambda_{f a c}} .
$$

If, in addition, $\tau_{f a c, 1} \neq \tau_{f a c, 2}$ and $\left|\tau_{f a c, 1}-\tau_{f a c, 2}\right|>0$ is large enough, then $\lambda_{f a c}=1$ and

$$
\Delta S_{n}^{\#}=X_{1, n} \frac{Z_{1, n} Z_{2, n}}{a_{f}^{2}}=\Delta S_{1, n} \frac{Z_{2, n}}{a_{f}} .
$$

- If $\tau_{f a c, 1}=\tau_{f a c, 2}$, then $Z_{1, n}=Z_{2, n}$ for all $n, \lambda_{f a c}=2$ and

$$
\Delta S_{n}^{\#}=\left[X_{1, n} X_{2, n}\right]^{\lambda_{d e p}} \frac{Z_{1, n}}{a_{f}} .
$$

If, in addition, $\tau_{d e p, 1} \neq \tau_{d e p, 2}$ and $\left|\tau_{d e p, 1}-\tau_{d e p, 2}\right|>0$ is large enough, then $\lambda_{d e p}=1$ and

$$
\Delta S_{n}^{\#}=X_{1, n} X_{2, n} \frac{Z_{1, n}}{a_{f}}=\Delta S_{1, n} X_{2, n} .
$$

- It follows that if both $\tau_{d e p, 1}=\tau_{d e p, 2}$ and $\tau_{f a c, 1}=\tau_{f a c, 2}$, then $X_{1, n}=X_{2, n}$ and $Z_{1, n}=Z_{2, n}$ for all $n, \lambda_{d e p}=\lambda_{f a c}=2$ and

$$
\Delta S_{n}^{\#}=X_{1, n} \frac{Z_{1, n}}{a_{f}}=\Delta S_{1, n}
$$

- If $\tau_{d e p, 2} \ll 1$ and $\left|\tau_{d e p, 1}-\tau_{d e p, 2}\right|$ is large enough, then $X_{2, n}=1$ for all $n$ (after a very short transient), $\lambda_{d e p}=1$, and then

$$
\Delta S_{n}^{\#}=X_{1, n}\left[\frac{Z_{1, n} Z_{2, n}}{a_{f}^{2}}\right]^{\lambda_{f a c}} .
$$

If, in addition, $\tau_{d e p, 1} \ll 1$ and $\tau_{d e p, 2} \sim \tau_{d e p, 1}\left(\left|\tau_{d e p, 1}-\tau_{d e p, 2}\right| \sim 0\right.$ not large enough), then $X_{1, n}=1$ for all $n$ (after a very short transient), $\lambda_{d e p}=2$, and then

$$
\Delta S_{n}^{\#}=\left[\frac{Z_{1, n} Z_{2, n}}{a_{f}^{2}}\right]^{\lambda_{f a c}} .
$$

- If $\tau_{f a c, 2} \ll 1$ and $\left|\tau_{f a c, 1}-\tau_{f a c, 2}\right|$ is large enough, then $Z_{2, n}=a_{f}$ for all $n$ (after a very short transient), $\lambda_{f a c}=1$, and then

$$
\Delta S_{n}^{\#}=\left[X_{1, n} X_{2, n}\right]^{\lambda_{d e p}} \frac{Z_{1, n}}{a_{f}} .
$$

If, in addition, $\tau_{f a c, 1} \ll 1$ and $\tau_{f a c, 2} \sim \tau_{f a c e, 1}\left(\left|\tau_{f a c, 1}-\tau_{f a c, 2}\right| \sim 0\right.$ not large enough), then $Z_{1, n}=a_{f}$ for all $n$ (after a very short transient), $\lambda_{f a c}=2$, and then

$$
\Delta S_{n}^{\#}=\left[X_{1, n} X_{2, n}\right]^{\lambda_{d e p}} .
$$

- It follows that if $\tau_{d e p, 1}, \tau_{d e p, 2} \ll 1\left(\left|\tau_{d e p, 1}-\tau_{d e p, 2}\right| \sim 0\right.$ not large enough) and $\tau_{f a c, 1}, \tau_{f a c, 2} \ll 1$ ( $\left|\tau_{f a c, 1}-\tau_{f a c, 2}\right| \sim 0$ not large enough), then

$$
\Delta S_{n}^{\#}=1 .
$$




\section{Supplementary Material}

\section{The Markran-Tsodyks (MT) model}

First, we briefly remark on the notation used in this section. We conduct analysis using continuous extensions of $\Delta S_{n}, S_{n}$, and $V_{n}$ - denoted as $\Delta S, S, V$ in the forthcoming figures and text. Time scales of temporal HPFs and LPFs in $S$ are denoted $\sigma_{f, S}$ and $\sigma_{d, S}$. The third time scale in temporal BPFs in $S$ are denoted $\sigma_{d+f, S}$. All temporal filters are fitted using gradient descent of a quadratic cost function.

The analysis for the MT model proceeds similarly to the DA models'. As seen in Section 3.3, the interaction between $X$ and $Z$ produces low-, high-, and band-pass temporal filters in $\Delta S$. Similarly, $R$ and $u$ produce low-, high-, and band-pass temporal filters in $\Delta S$. Again, we find $\Delta S$ temporal LPFs and HPFs not only develop in synapses exhibiting exclusively STD and STF, respectively. Indeed, $\Delta S$ temporal LPFs (HPFs) can develop in synapses where the time scale of depression (facilitation) dominates facilitation (depression). However, as in the case of the DA model, the exact ranges of $f_{s p k}$ over which LPFs and HPFs develop depend on the balance between facilitation and depression. Figure S1-A1 shows that almost exclusively depressive synapses exhibit LPFs for $0<f_{s p k}<150$, whereas a dominantly depressive synapse may stop producing LPFs for $f_{s p k}>100$ (compare to Figure S1-A5). A similar situation arises in facilitating synapses (Figure S1-A2 and -A5).

$R_{n}$ and $u_{n}$ are well described by exponential decays, much in the same way $X_{n}$ and $Z_{n}$ are observed to be (Section 3.4). As such, as we did in the DA model, in the MT model we imagine that temporal filters in $\Delta S$ are heuristically the product of two exponentials. Despite the non-linearity present in MT model which complicates the analysis of how the long-term time scales of $R_{n}$ and $u_{n}$ are passed through their product $\Delta S, \Delta S$ temporal LPFs (examples in Figures S2-B2,-B3) and HPFs (examples in Figures S2A2,-A3) are still well described by a single time scale exponential. The time scales extracted from HPFs at dominantly facilitating and exclusively facilitating synapses are summarized in Figure S2-A1. Figure S2-B shows analogous results for LPFs of the MT model. A careful reader will note that Figure S2 refers to temporal filters of $S$, rather than $\Delta S$. However, $\tau_{d e c}=3$ for these figures so that the contribution of the synaptic HPF implemented by synaptic decay is inconsequential for this discussion. The same remark also applies to Figures S3, S6, and S7.

In Section 3.6, BPFs in the DA model are shown to arise from 3 time scales, 2 of which can be extracted from corresponding LPFs and HPFs. A similar result is true for the MT model. Figure S3 outlines how these three time scales vary as the input frequency varies. Band-pass temporal filters become more sharply peaked as the input frequency increases. This reflects itself as observable decreases in the scenario where three time scales characterize band-pass temporal filters. Figure S3-B show that this third time scale is not superfluous - that removing the $\sigma_{d+f, S}$ time scale from the model drastically alters the temporal filter fit.

Finally, we briefly review how $\Delta S$ temporal LPFs, HPFs, and BPFs are propagated to $S$ and PSP. We note that $\tau_{d e c}$ and $\tau_{m e m}$ implement temporal HPFs. Figure $\mathrm{S} 4$ summarizes the types of filters that result in $S$ from different incident $\Delta S$ temporal filters. Similarly, Figure S5 summarizes the types of filters that result in $V$ from different incident $S$ temporal filters. As in the DA model, we note there are instances where BPFs are passed through levels of organization and others where they arise due to an interaction of filters at different levels of organization (see Section 3.7). The communication through BPFs between levels of organization is exemplified by Figure S4-A3 $(\Delta S$ to $S$ ) and Figure S5-A3 $(S$ to $V)$. BPFs arising from interactions of filters between levels of organization are exemplified by Figure S4-A1 $(\Delta S$ to $S)$ and Figure S5-A1 ( $S$ to $V$ ).

BPFs in the PSP arise in two ways - either passed through from the incident BPF filter or as the result of interacting an incident LPF and HPF implemented by the post-synaptic cell. These BPFs can be distinguished by analyzing the three time constants used to fit BPFs. First we consider synaptic BPFs that transfer to the post-syntactic cell's response.

In this case, the procedure to extract the three time constants of the PSP BPF differs slightly from the 

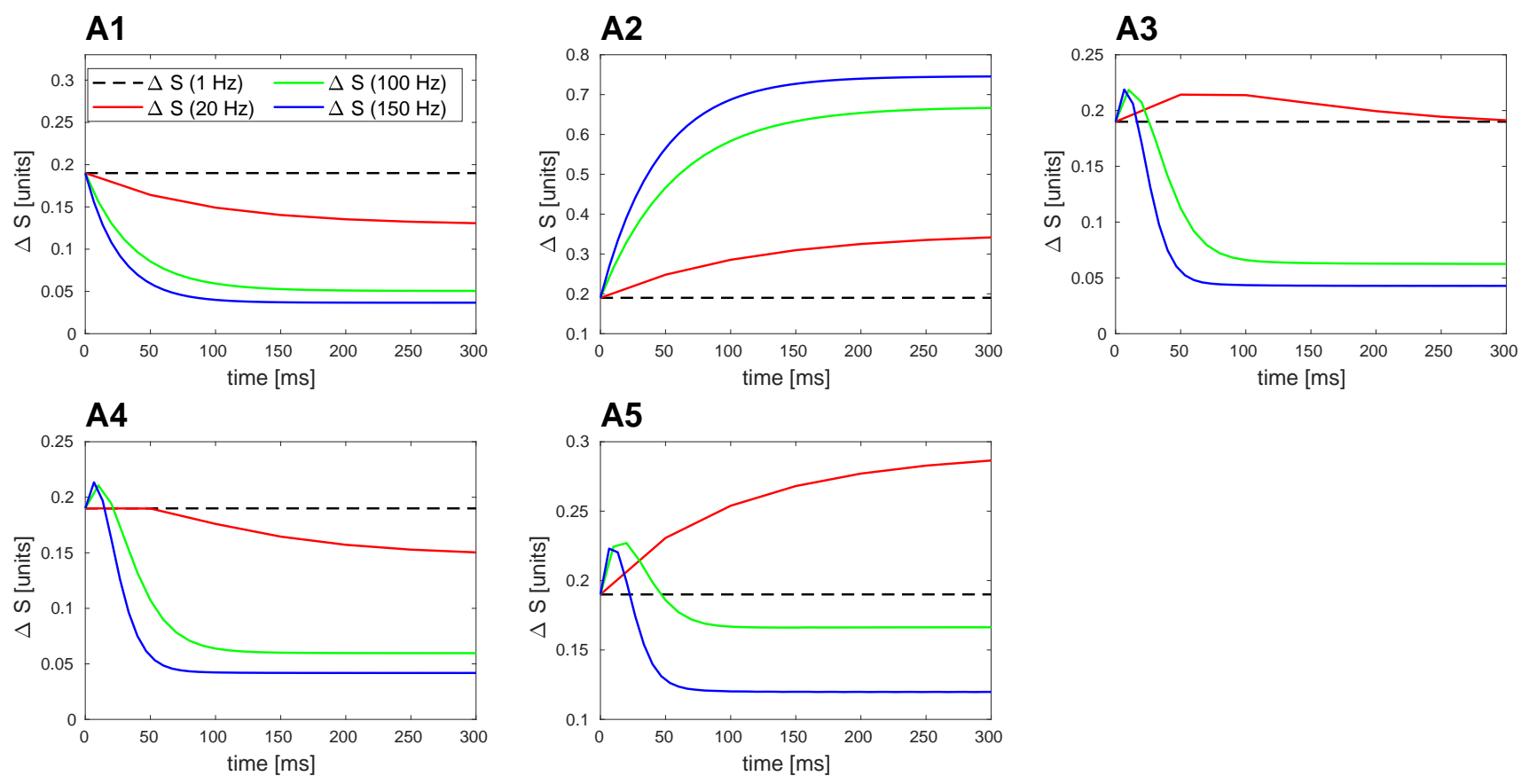

Figure S1: Temporal Filters in $\Delta S$ for the MT model. The interaction of presynaptic spiking and STP timescales create only high-pass, low-pass, and band-pass temporal filters. For these simulations $\tau_{d e c}=$ .01 to suppress summation. A1. Low-pass temporal filters appear for all input frequencies. As the input frequency increases, the low-pass temporal filters decay more aggressively $\left(\tau_{d e p}=150, \tau_{f a c}=1\right)$. A2. High-pass temporal filters appear for all input frequencies. As the input frequency increases, the high-pass temporal filters rise more aggressively $\left(\tau_{d e p}=1, \tau_{f a c}=150\right)$. A3. Band-pass temporal filters appear for all input frequencies. As the input frequency increases, the band-pass temporal filters become more sharply peaked $\left(\tau_{d e p}=150, \tau_{f a c}=150\right)$. A4. Low-pass temporal filters appear for low input frequencies but then band-pass temporal filters develop for higher input frequencies $\left(\tau_{d e p}=150, \tau_{f a c}=30\right)$. A5. High-pass temporal filters appear for low input frequencies but then band-pass temporal filters develop for higher input frequencies $\left(\tau_{d e p}=30, \tau_{f a c}=150\right)$. In all simulations for the synapse: $U_{0}=.1$. 


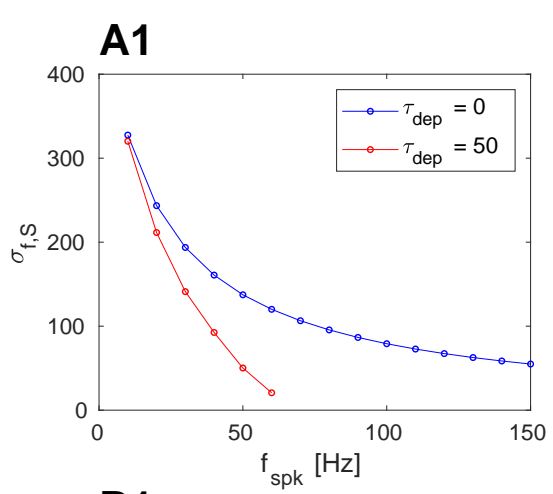

B1

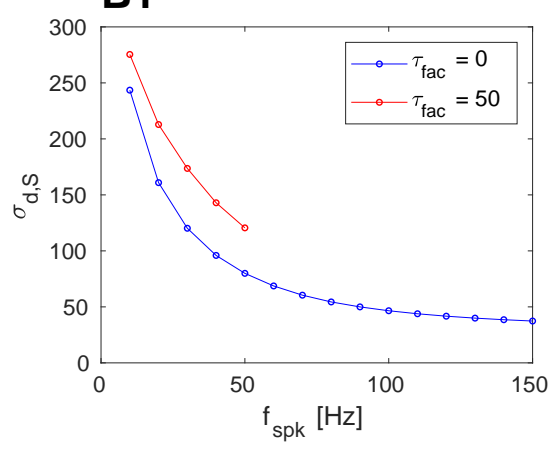

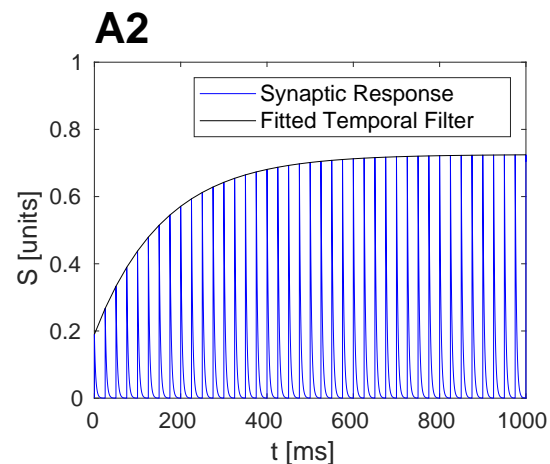

B2

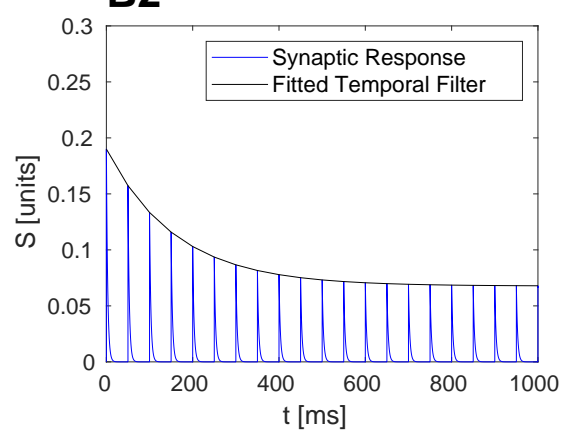

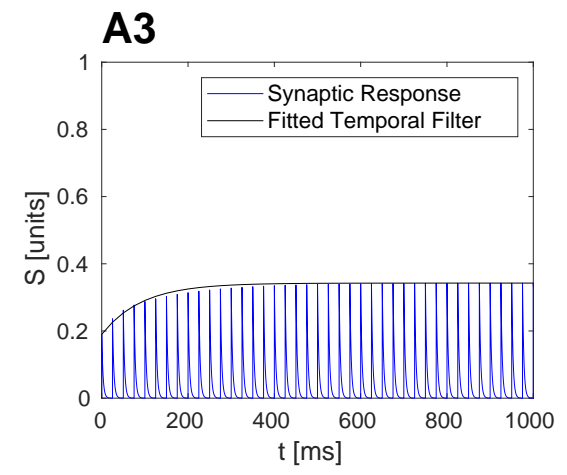

B3

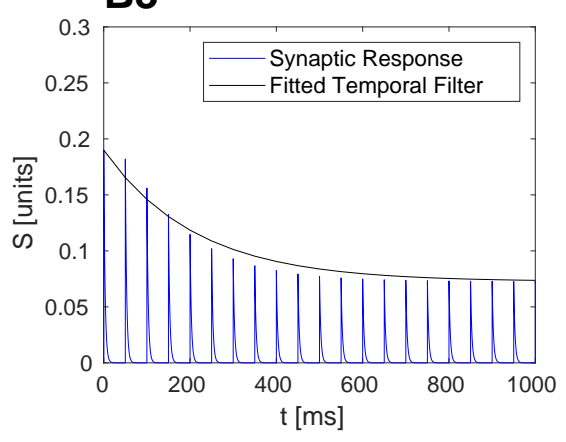

Figure S2: A. STF dominated synapses exhibit high-pass temporal filter. $\left(\tau_{f a c}=500\right)$ A1. The dependence of high-pass temporal filter's time scale on input frequency: a comparison of a synapse with no STD and fast STD. A2. Example of high-pass temporal filter at synapse with no STD. $\left(\tau_{d e p}=0, f_{s p k}=40\right)$ A3. Example of high-pass temporal filter at synapse with fast STD. $\left(\tau_{d e p}=50, f_{s p k}=40\right)$ B. STD dominated synapses exhibit low-pass temporal filter: a comparison of a synapse with no STF and fast STF. $\left(\tau_{d e p}=500\right)$ B1. The dependence of low-pass temporal filter's time scale on input frequency. B2. Example of low-pass temporal filter at synapse with no STF. $\left(\tau_{f a c}=0, f_{s p k}=20\right)$ B3. Example of low-pass temporal filter at synapse with fast STF. $\left(\tau_{f a c}=50, f_{s p k}=20\right)$ In all simulations for the synapse: $U_{0}=.1$ and $\tau_{d e c}=3$. Upper bound of RMSE on all low- and high-pass temporal fits: .012. 

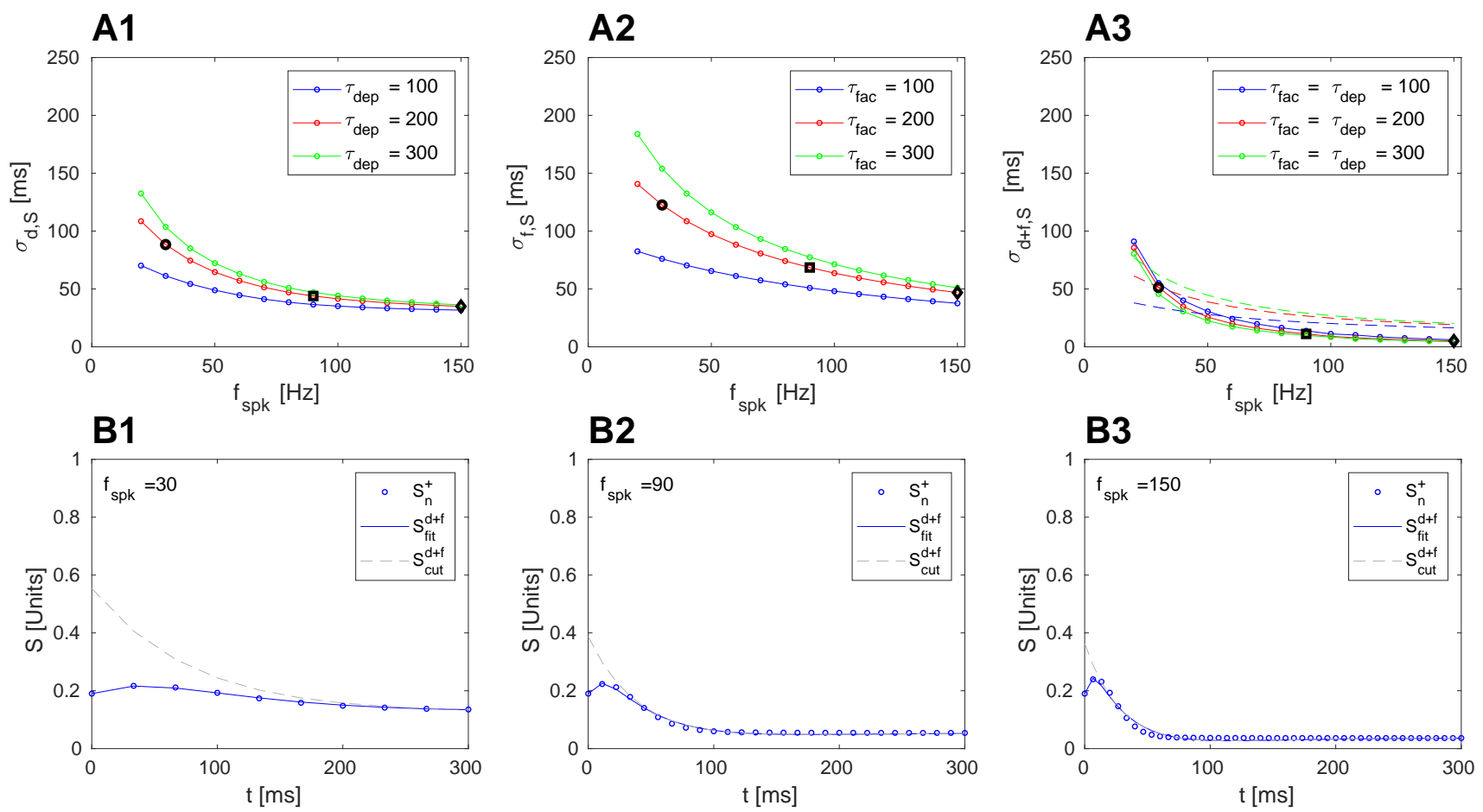

Figure S3: The time scales of band-pass temporal filters are determined by the mix of STF's and STD's time scales at the synapse along with input frequency. A. The time scales of STD and STF impact the three time scales characterizing band-pass filters in different ways. A1. The impact of STD's time scale, $\tau_{d e p}$, on the band-pass temporal filter's time scale related to low-pass temporal filters. $\left(\tau_{f a c}=0\right)$ A2. The impact of STF's time scale, $\tau_{f a c}$, on the band-pass temporal filter's time scale related to high-pass temporal filters. ( $\left.\tau_{d e p}=0\right)$ A3. The impact that both STD's and STF's time scale have on third time scale of temporal filtering. Solid lines are fit $\sigma_{d+f, S}$ fitted from three time scale model of temporal BPFs. The dashed lines are the corresponding values of $\left(1 / \sigma_{d}+1 / \sigma_{f}\right)^{-1}$. B. The third time scale is not redundant. Without it, the temporal band-pass filter will fail to fit. $\left(\tau_{d e p}=\tau_{f a c}=200\right)$. The three time scales for the temporal filters in the following figures can be obtained from the foregoing three figures. $S_{f i t}^{d+f}$ is the temporal BPF extracted using the three time scale model. $S_{\text {cut }}^{d+f}$ is obtained by setting the coefficient on the third time scale to zero. B1. Temporal BPF fit with and without third time scale when $f_{s p k}=30$. The circles in A represent the time scales extracted using the three time scale model BPF model. B2. Temporal BPF fit with and without third time scale when $f_{s p k}=90$. The squares in A represent the time scales extracted using the three time scale model BPF model. B3. Temporal BPF fit with and without third time scale when $f_{s p k}=150$. The diamonds in A represent the time scales extracted using the three time scale model BPF model In all simulations for the synapse: $U_{0}=.1$ and $\tau_{d e c}=3$. Upper bound of RMSE on all temporal filter fits used in this figure: .01 . 

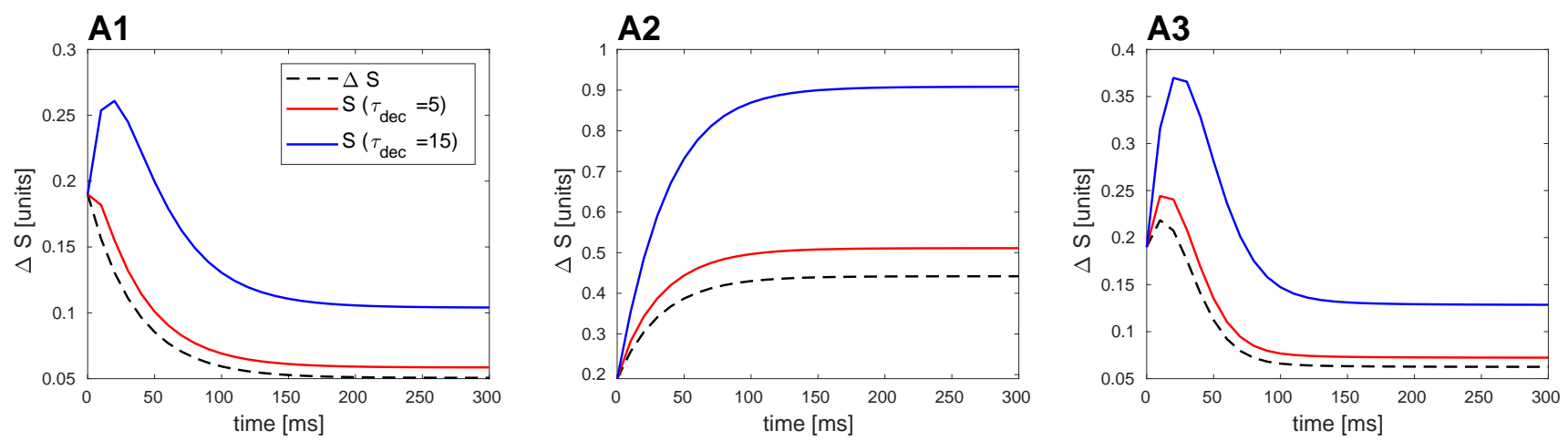

Figure S4: Temporal Filters in $S$ for the MT model. $\Delta S$ create only low-pass, high-pass, and band-pass temporal filters. Now we examine the effect that summation has on these temporal filters. The effect of summation can be understood to be a high-pass temporal filter interacting with a temporal filter created from the interaction of input and STP time scales (shown here for $f_{s p k}=100$ ). A1. The interaction of a low-pass temporal filter in $\Delta S$ with the time scale of summation, $\tau_{d e c}$ is shown. We observe that low-pass temporal filters become band-pass temporal filters for longer time scales of summation $\left(\tau_{\text {dep }}=150, \tau_{f a c}=1\right)$. Note that for extreme, unphysiological time scales of summation $\left(\tau_{d e c}>150\right)$, high pass temporal filters in $S$ may also develop (not shown). A2. The interaction of a high-pass temporal filter in $\Delta S$ with the time scale of summation, $\tau_{d e c}$ is shown. We observe that high-pass temporal filters remain high-pass temporal filters for any time scale of summation $\left(\tau_{d e p}=1, \tau_{f a c}=150\right)$. A3. The interaction of a band-pass temporal filter in $\Delta S$ with the time scale of summation, $\tau_{d e c}$ is shown. We observe that band-pass temporal filters remain band-pass temporal filters $\left(\tau_{d e p}=150, \tau_{f a c}=150\right)$. Longer time scales of summation increase the size of the band-pass peak (both in height and width). Note that for extreme, unphysiological time scales of synaptic decay $\left(\tau_{\text {dec }}>150\right)$, high pass temporal filters in $S$ may also develop (not shown). In all simulations for the synapse: $U_{0}=.1$. 

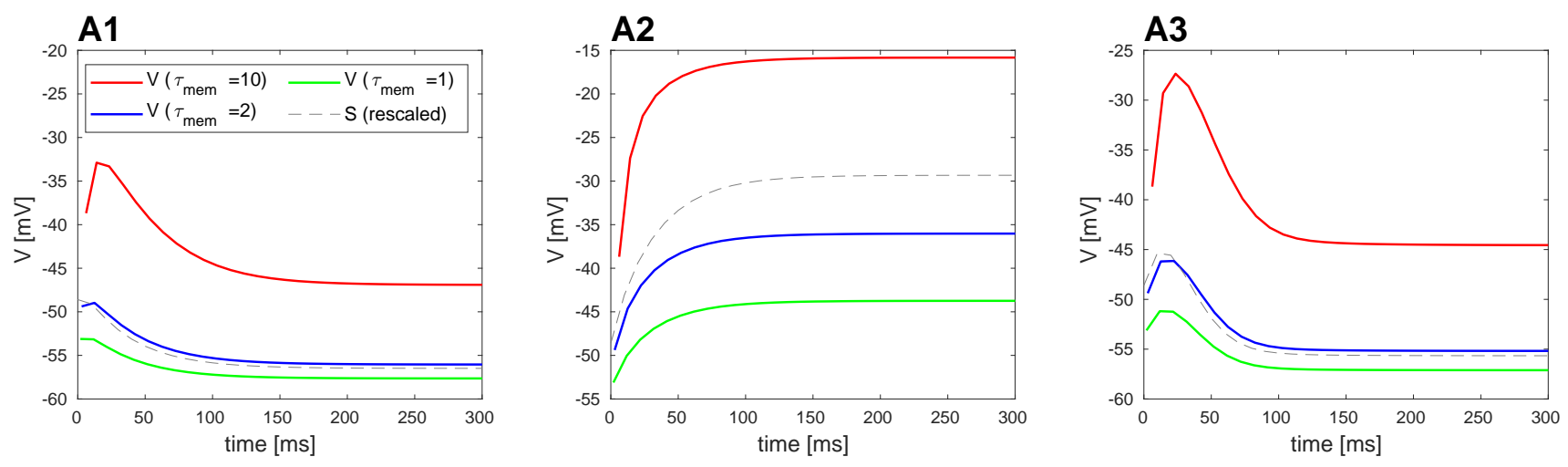

Figure S5: Temporal Filters in $V$ for the MT model. The interaction of input, STP, and summation time scales combine to create only low-pass, high-pass, and band-pass temporal filters. Now we examine the effect that the membrane time constant of the post-synaptic cell has on the temporal filters incident from the synapse. For fast enough membrane time constants $\left(\tau_{m e m}<1 \mathrm{~ms}, g_{L}>1\right)$ the post-synaptic temporal filter reflects the synaptic temporal filter $\left(f_{s p k}=100, \tau_{d e c}=5\right)$. As the membrane time constant slows, the synaptic temporal filter interacts with the post-synaptic cell. Biophysically, the effect of a slow membrane time constant is to create post-synaptic summation - resulting in a high-pass post-synaptic temporal filter. The development of an interaction between the high-pass post-synaptic temporal filter with the synaptic temporal filter is seen in these figures as the membrane time constant slows. For these figure $G_{L}=0.1,0.5$, and 1 . These correspond to $\tau_{m e m}=10 \mathrm{~ms}, 2 \mathrm{~ms}$, and $1 \mathrm{~ms}$, respectively. A1. The interaction of a low-pass synaptic temporal filter in $S$ (rescaled and shown in gray) with the membrane time constant is shown. We observe that low-pass synaptic temporal filters can become band-pass temporal filters as the membrane time constant slows $\left(\tau_{d e p}=150, \tau_{f a c}=1\right)$. A2. The interaction of a high-pass synaptic temporal filter in $S$ (rescaled and shown in gray) with the membrane time constant is shown. We observe that high-pass synaptic temporal filters remain high-pass temporal filters in the post-synaptic response as the membrane time constant slows $\left(\tau_{d e p}=1, \tau_{f a c}=150\right)$. A3. The interaction of a band-pass synaptic temporal filter in $S$ (rescaled and shown in gray) with the membrane time constant is shown. We observe that band-pass synaptic temporal filters remain band-pass temporal filters in the post-synaptic cell $\left(\tau_{d e p}=150, \tau_{f a c}=150\right)$. In particular, as the membrane time constant slows, the size of the band-pass peak increases (both in height and width). We remark that for all cases, for extremely slow membrane time constants $\left(\tau_{m e m}>1\right.$ sec or $g_{L}<.001$ ), high-pass post-synaptic temporal filters develop (not shown). In all simulations for the synapse: $U_{0}=.1, \tau_{d e c}=5$. In all simulations of the post-synaptic cell: $G_{e x}=1, C=1, E_{L}=-60, E_{e x}=0$. 
procedure used to extract the three time constants of BPFs in $S$ and $\Delta S$. Instead of fixing LPF and HPF time scales and finding the best fitting third time constant, as was done in the $\Delta S$ BPFs, all three time constants for the PSP BPF are allowed to vary. In this way, we find a triple of time constants that fit the PSP BPF: $\rho_{a}, \rho_{b}$ and $\rho_{c}$ (Example shown in Figure S6-A3). Then we introduce the following quantities:

$$
\begin{gathered}
\rho_{1} \quad=\max \left(\rho_{a}, \rho_{b}, \rho_{c}\right) \\
\rho_{2}=\operatorname{median}\left(\rho_{a}, \rho_{b}, \rho_{c}\right) \\
\rho_{3} \quad=\min \left(\rho_{a}, \rho_{b}, \rho_{c}\right)
\end{gathered}
$$

These time constants are then compared to the magnitude of the time constants obtained from the synaptic BPF. In Figure S6-C we note that the synaptic BPF has time constants such that $\sigma_{d, S}>\sigma_{f, S}>\sigma_{d+f, S}$. Assuming the post-synaptic cell maintains this relation, we associate $\rho_{1}$ with $\sigma_{d, S}, \rho_{2}$ with $\sigma_{f, S}$, and $\rho_{3}$ with $\sigma_{d+f, S}$. Figure S6-C suggests that the HPF implemented by the membrane time constant is modifying all three time constants of the incident synaptic BPF.

BPFs in the post-synaptic cell are implemented also by the interaction with synaptic LPFs and the HPF implemented by the membrane time constant. One may use the same three parameter model to fit these PSP BPFs. However, we find that there are actually two time scales describing the shape of these BPFs. Figure S7-B show explicit examples of the third time scale's insignificance. Furthermore, one of the time scales describing the PSP BPF is shown to be inherited from the incident LPF's time scale of decay.

Figure -B1 and -B2 show how the membrane time constant also modifies incident LPF and HPF time constants in the post-synaptic cell. Here, the same models to extract time constants of rise for HPFs and time constants of decay for LPFs in $\triangle S$ also work well for extracting time constants for LPFs and HPFs of the PSP (example fits in Figure S6-A1 and -A2). In this figure, $\sigma_{f, V}$ is the time constant of rising in PSP HPFs and $\sigma_{d, V}$ is the time constant of decay in PSP LPFs, respectively.

In Section 3.9.2, we discuss how the temporal filters implemented by $Z_{n}, X_{n}$, and $\Delta S_{n}$ persist in the presence of Poisson spiking. Here we review a potential consequence of Poisson inputs interacting with temporal filters: gain control. The data in the following figure, Figure S8, uses the MT model, however, the foregoing discussion suggests that the DA and MT model both exhibit temporal filters, albeit via slightly differing quantitative mechanisms. Figure $S 8$ plots average amplitude of voltage response over 1100 trials where the input Poisson rates change over time. As the spiking rates change over time, different steady states are achieved. Overshooting transient features develop between rate changes when STD time scales increase. The fact that these overshoots depend on the time scale of STD suggests that there may be a connection between the overshoot magnitudes and the LPFs that STD implement. Furthermore, the magnitude of these average rate changes and their dependence on the initial and final rates was studied as a mechanism for gain control by Abbott et. al.. Figure S8 show that average amplitude also depends on the time scale of STD and membrane time scales - ergo, it follows that filters that STD and membrane time constants implement may also play an important role in determining the average amplitude of voltage response. 


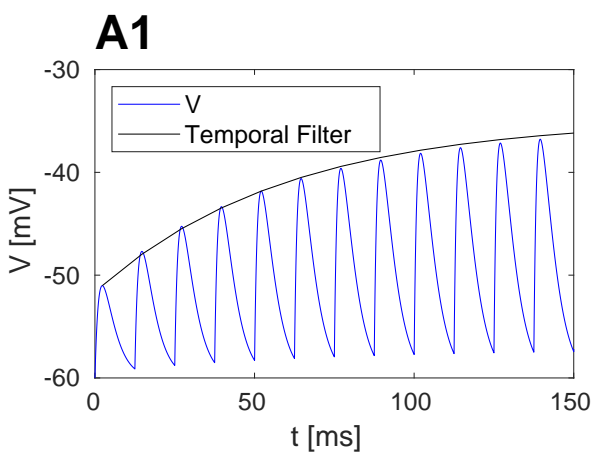

B1
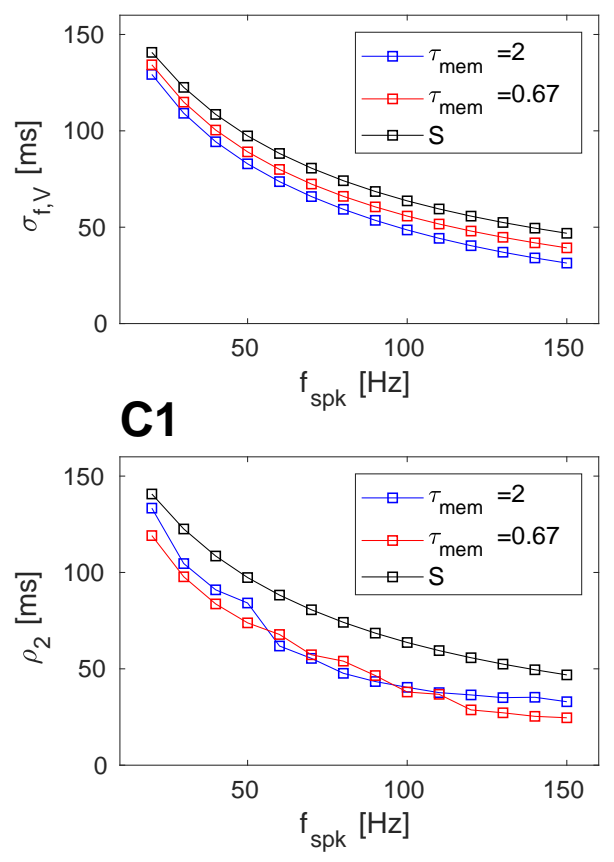

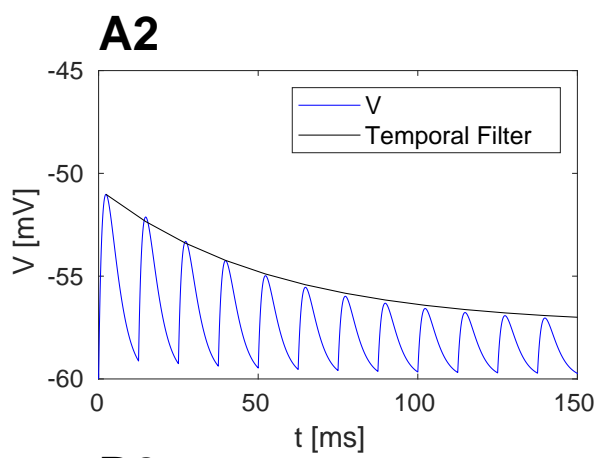

B2
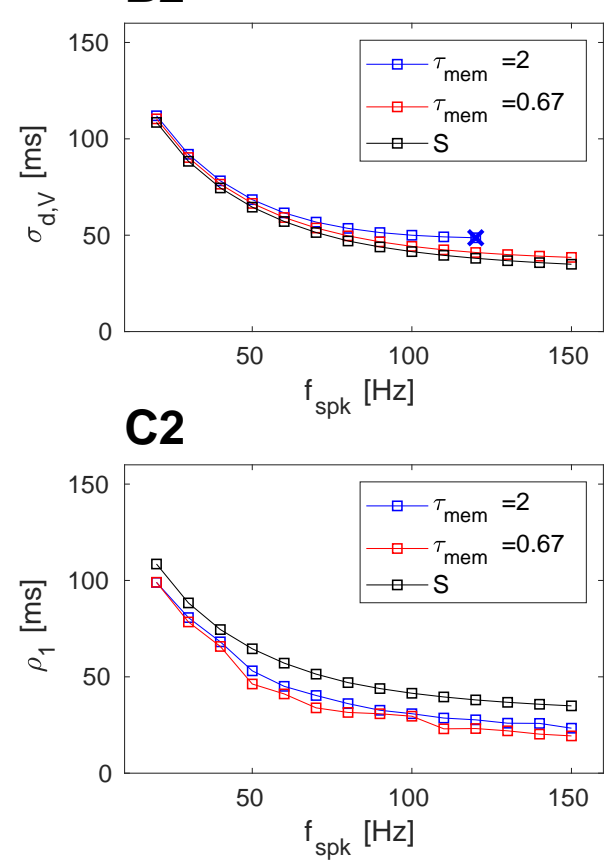
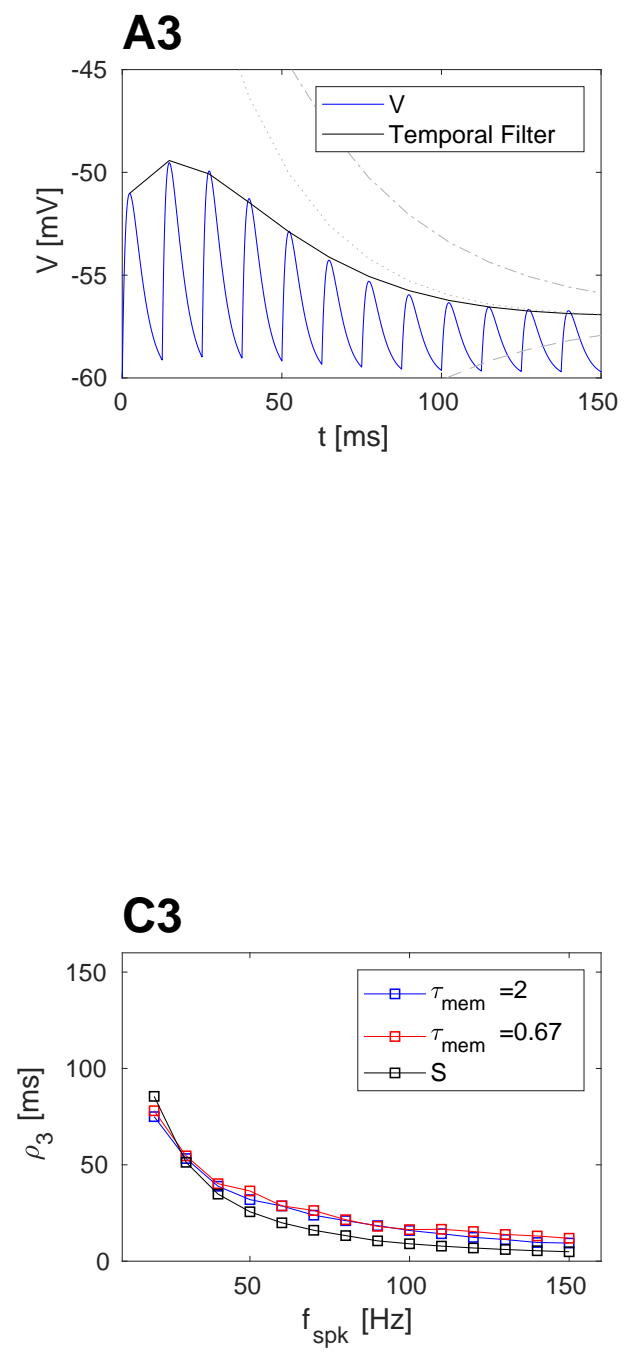

Figure S6: A. Representative fits of low-, high-, and band-pass temporal filters in passive post-synaptic cell. $\left(G_{L}=.5\right)$ A1. Example of high-pass post-synaptic temporal filter. $\left(\tau_{d e p}=0, \tau_{f a c}=200, f_{s p k}=80\right) \mathbf{A 2}$. Example of low-pass post-synaptic temporal filter. $\left(\tau_{d e p}=200, \tau_{f a c}=0, f_{s p k}=80\right)$ A3. Example of bandpass post-synaptic temporal filter. $\left(\tau_{d e p}=200, \tau_{f a c}=200, f_{s p k}=80\right)$ B1. The impact that membrane time constant has on the time scale of post-synaptic high-pass temporal filters. The incident synaptic temporal filter is high-pass and given by $\tau_{d e p}=0, \tau_{f a c}=200$. B2. The impact that membrane time constant has on the time scale of post-synaptic low-pass temporal filters. The incident synaptic temporal filter is low-pass and given by $\tau_{d e p}=200, \tau_{f a c}=0$. "X" marks the input frequency at which the post-synaptic temporal filter transitions from low-pass to band-pass. These band-band pass temporal filters are analyzed in Figure S7. C. The impact that membrane time constant has on the time scales of post-synaptic band-pass temporal filters. The incident synaptic temporal filter is band-pass and given by $\tau_{d e p}=200, \tau_{f a c}=200$. The way the time scales, $\rho_{1}, \rho_{2}$ and $\rho_{3}$, are obtained are outlined in Methods. C1. The impact of the membrane time constant on $\rho_{1}$. C2. The impact of the membrane time constant on $\rho_{2}$. $\mathbf{C} 3$. The impact of the membrane time constant on $\rho_{3}$. All simulations were performed using MT Model with $U_{0}=.1$ and $\tau_{d e c}=3$. The parameters for the passive cell are $G_{e x}=1, C=1, E_{L}=-60, E_{e x}=0$. The upper bound on the RMSE for all temporal filters (low-, high-, and band-pass) is .4 mV. The upper bound on the maximum difference between a fit and the temporal filters for all voltage responses is $1 \mathrm{mV}$. 


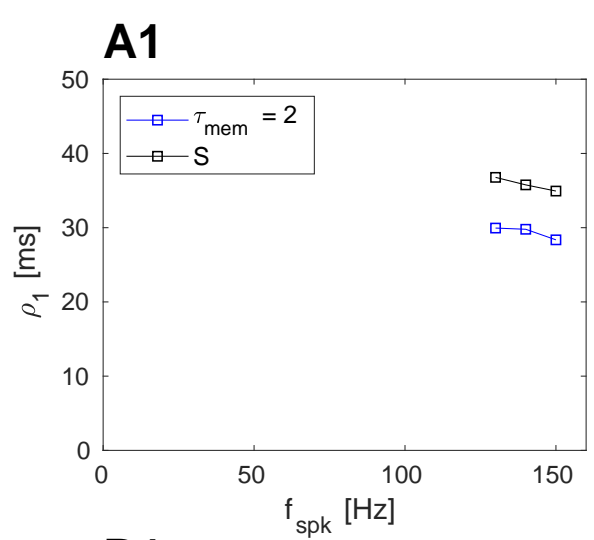

B1

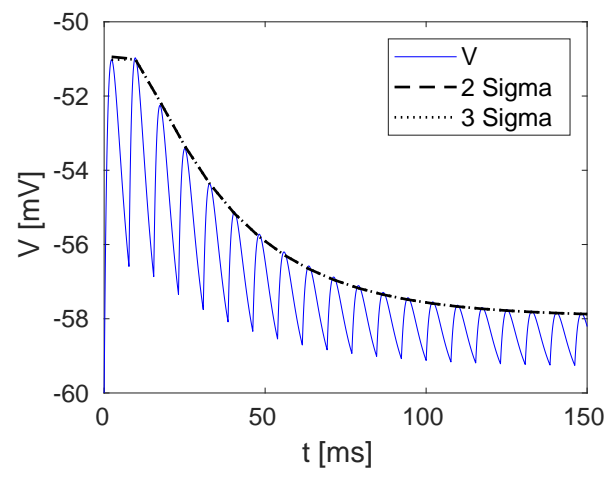

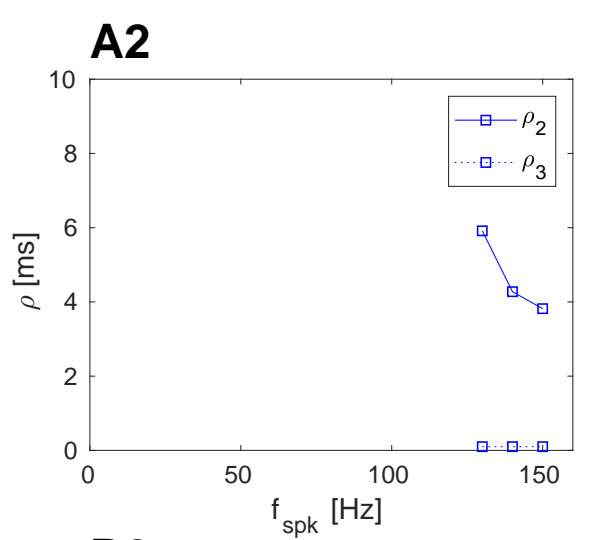

B2

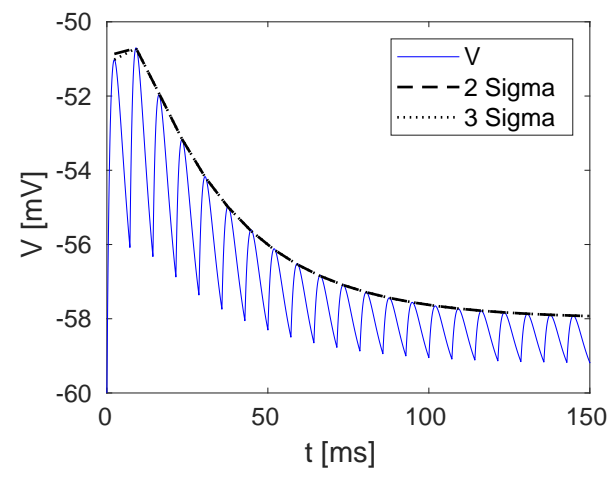

B3

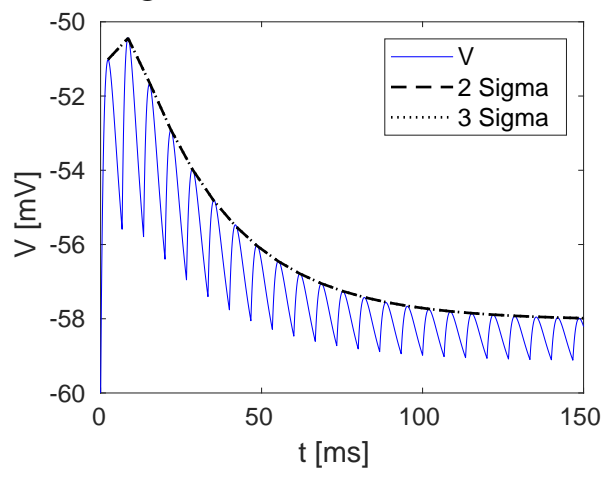

Figure S7: A. Time scales of band-pass temporal filters formed by incident low-pass synaptic temporal filters. The incident synaptic temporal filter is low-pass and given by $\tau_{d e p}=200, \tau_{f a c}=0$. A1. $\rho_{1}$ plotted as a function of frequency and compared to $\sigma_{d}$. A2. $\rho_{2}$ and $\rho_{3}$ plotted as a function of frequency. B. The three temporal band-pass temporal filters plotted in A. "3 sigma" is the fit obtained using BPF filter model letting all three time scales vary. "2 sigma" is the fit obtained omitting the fastest time scale, $\rho_{3}$, from the fit. $\left(G_{L}=.4\right)$ B1. Temporal band-pass temporal filters plotted in A with its fits. $\left(f_{s p k}=130\right)$ B2. Temporal band-pass temporal filters plotted in A with its fits. $\left(f_{s p k}=140\right)$ B3. Temporal band-pass temporal filters plotted in A with its fits. $\left(f_{s p k}=150\right)$ All simulations were performed using MT Model with $U_{0}=.1$ and $\tau_{d e c}=3$. The parameters for the passive cell are $G_{e x}=1, C=1, E_{L}=-60, E_{e x}=0$. The upper bound on the RMSE for all temporal filters (low-, high-, and band-pass) is .4 mV. The upper bound on the maximum difference between a fit and the peaks of voltage response is $1 \mathrm{mV}$. 


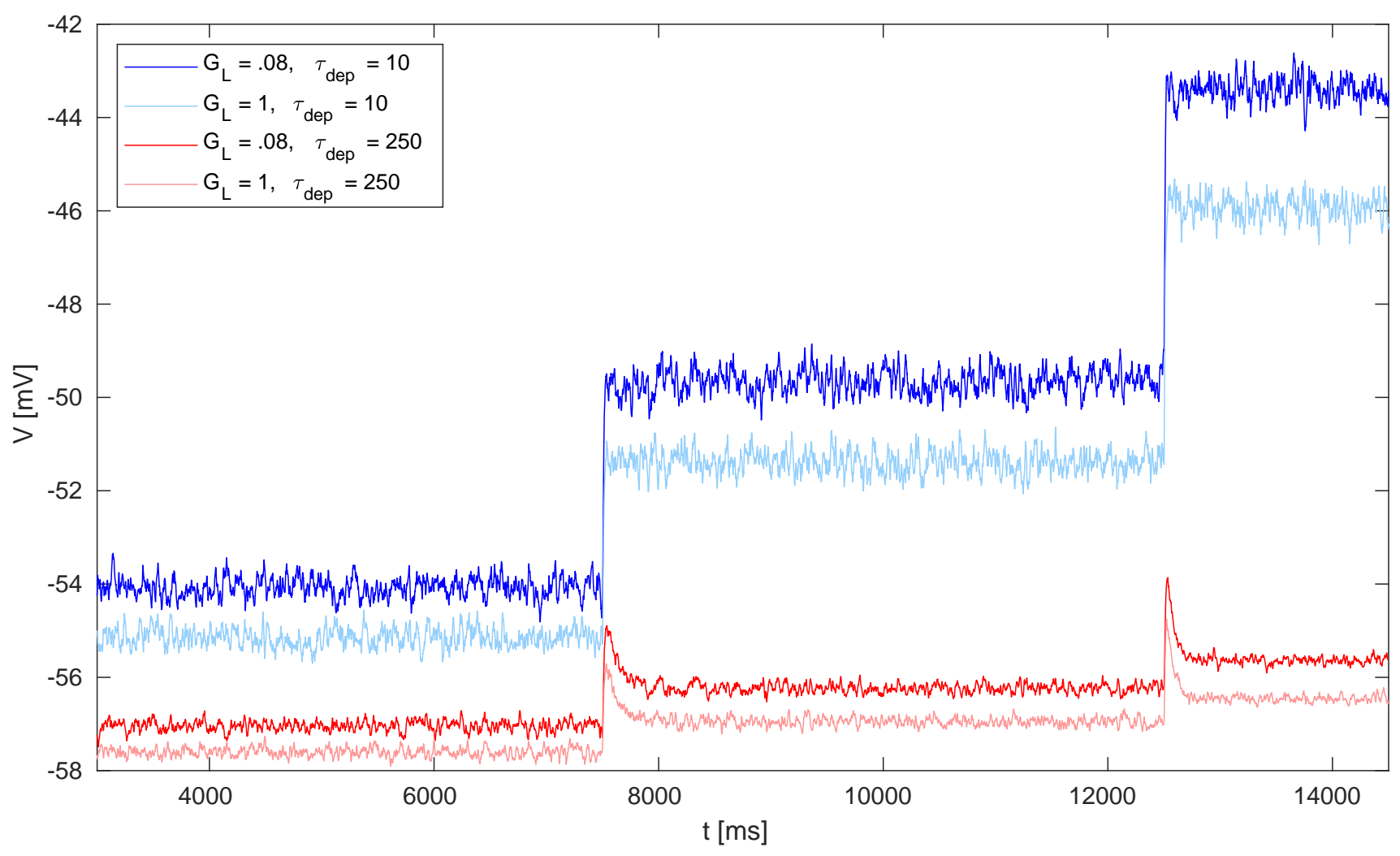

Figure S8: Each trace in the figure is the average of 1100 trails. A trial consists of the following: 7.5 secs of $20 \mathrm{~Hz}$ Poisson stimulus, 5 secs of $40 \mathrm{~Hz}$ Poisson stimulus, and 2 secs of $80 \mathrm{~Hz}$ Poisson stimulus. The first second of the simulation is cut off to remove the transient behaviors from the initialization of the simulation. All simulations were performed using MT model with parameters: $\tau_{f a c}=0, \tau_{d e c}=3, U_{0}=.1$. Passive post-synaptic cell parameters were $C=1, G_{e x}=.1, E_{L}=-60, E_{e x}=0$. 